\title{
Supersymmetric gauged double field theory: systematic derivation by virtue of twist
}

\author{
Wonyoung Cho, ${ }^{a}$ J.J. Fernández-Melgarejo, ${ }^{b}$ Imtak Jeon ${ }^{c}$ and Jeong-Hyuck Park ${ }^{a}$ \\ ${ }^{a}$ Department of Physics, Sogang University, \\ Mapo-gu, Seoul, 121-742 Korea \\ ${ }^{b}$ Department of Physics, Harvard University, \\ Cambridge, MA, 02138 U.S.A. \\ ${ }^{c}$ Korea Institute for Advanced Study, \\ Dongdaemun-gu, Seoul, 130-722 Korea \\ E-mail: ycho@sogang.ac.kr, josejuan@physics.harvard.edu, \\ imtakjeon@kias.re.kr, park@sogang.ac.kr
}

ABSTRACT: In a completely systematic and geometric way, we derive maximal and halfmaximal supersymmetric gauged double field theories in lower than ten dimensions. To this end, we apply a simple twisting ansatz to the $D=10$ ungauged maximal and halfmaximal supersymmetric double field theories constructed previously within the so-called semi-covariant formalism. The twisting ansatz may not satisfy the section condition. Nonetheless, all the features of the semi-covariant formalism, including its complete covariantizability, are still valid after the twist under alternative consistency conditions. The twist allows gaugings as supersymmetry preserving deformations of the $D=10$ untwisted theories after Scherk-Schwarz-type dimensional reductions. The maximal supersymmetric twist requires an extra condition to ensure both the Ramond-Ramond gauge symmetry and the 32 supersymmetries unbroken.

KeYwords: Supergravity Models, Supersymmetry and Duality, Classical Theories of Gravity, String Duality

ARXiv EPrint: 1505.01301 


\section{Contents}

1 Introduction 1

2 The semi-covariant formulation of ungauged DFT 4

2.1 Coordinate gauge symmetry, section condition and diffeomorphism 5

$\begin{array}{ll}2.2 & \text { Dilaton, vielbeins and projectors }\end{array}$

$\begin{array}{lll}2.3 & \text { Semi-covariant derivatives, curvatures and their complete covariantizations } & 7\end{array}$

2.4 Fermions, Ramond-Ramond cohomology and completely covariant Dirac operators

$\begin{array}{lll}2.5 & \text { DFT action and supersymmetric extensions } & 18\end{array}$

3 U-twisted double field theory $\quad 20$

3.1 Ansatz for U-twist 20

3.2 Properties of the U-derivative and its connection 22

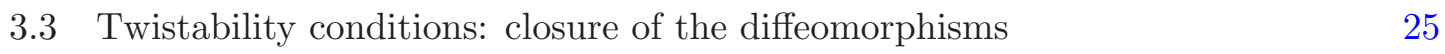

$\begin{array}{ll}3.4 & \text { Twisted semi-covariant formalism } \\ \end{array}$

4 Twisted supersymmetric double field theory $\quad 35$

4.1 Half-maximal supersymmetric gauged double field theory 35

4.2 Maximal supersymmetric gauged double field theory 36

$\begin{array}{lll}4.3 & \text { Explicit comparison with the untwisted case } & 37\end{array}$

$\begin{array}{lll}5 & \text { Discussion } & 39\end{array}$

\section{Introduction}

A characteristic of Double Field Theory (DFT) [1-4] is the section condition, a second order differential constraint imposed on arbitrary fields and their products, such that the $\mathbf{O}(D, D)$ invariant Laplacian should be trivial,

$$
\partial_{A} \partial^{A} \sim 0
$$

While DFT employs doubled spacetime coordinates [5-7] manifesting the $\mathbf{O}(D, D)$ structure of T-duality, the section condition ensures that DFT lives not on the doubled $(D+D)$ dimensional space but on a $D$-dimensional null hyperspace, i.e. section.

The geometric insight behind the section condition was proposed in [8] to claim that the coordinate space in DFT is doubled yet gauged: a gauge orbit rather than a point in the doubled coordinate space corresponds to a physical point. Within this picture, the exponentiation of the generalized Lie derivative which is the infinitesimal DFT-diffeomorphism generator was shown in [8] to agree with the then-known simple ansatz of the tensorial finite 
diffeomorphism à la Hohm and Zwiebach [9], cf. [10-14]. This 'coordinate gauge symmetry' was also soon successfully realized on a string worldsheet as a usual gauge symmetry [10] (cf. [15-17]), where the spacetime coordinates are dynamical fields. The constructed string action couples to an arbitrarily curved generalized metric and is still completely covariant with respect to the coordinate gauge symmetry, DFT-diffeomorphisms, world-sheet diffeomorphisms, world-sheet Weyl symmetry and $\mathbf{O}(D, D)$ T-duality. While it reduces to the conventional string action upon the Riemannian parametrization of the generalized metric, it can also go beyond the Riemannian regime. In this way, DFT is a stringy gravitational theory which is defined self-consistently adopting the doubled-yet-gauged coordinate system.

On the other hand, somewhat contrary to the geometric significance of the section condition, it has been also observed that, in order to correctly reproduce a variety of the known gauged supergravities in lower than ten-dimensions it is necessary to consider "relaxing" the section condition. In the supergravity literature, a powerful way of the gauging has been the embedding tensor method [18] which allows for a systematic classification of all possible supersymmetric deformations as for gaugings. However, while some of the gaugings can be obtained by a Scherk-Schwarz dimensional reduction of the eleven- or tendimensional supergravities [19, 20], a class of gaugings has been known to have no such a higher dimensional origin. This mystery got a new spin when Geissbühler [21] (cf. [22]) realized the necessity of introducing section-condition-breaking terms for DFT to reproduce the complete classification of the deformations of $N=4, D=4$ supergravity [23]. The section condition was broken by terms which depend on both the ordinary and the dual coordinates of the internal manifold, cf. [24]. This was an indication that DFT may go beyond the ordinary supergravity or Generalized Geometry [25, 26]. Possible modifications of the section condition were soon investigated by Grana and Marques [27] who looked for a set of consistency conditions for the closure of the generalized Lie derivative twisted by the Scherk-Schwarz ansatz. Since then there have been a few proposals made toward the underlying geometric principle, notably the flux formulation [28] and the torsionful deformation of the semi-covariant formalism by Berman and Lee [29].

In the flux formulation of DFT [28, 30,31], the basic building blocks constituting the Lagrangian are 'fluxes' which are diffeomorphic scalars, as in e.g. [1, 32, 33]. Yet, they are not local Lorentz covariant. The $\mathbf{S p i n}(1, D-1)_{L} \times \mathbf{S p i n}(D-1,1)_{R}$ local Lorentz symmetry is only forced at the whole action level. On the other hand, in the semi-covariant formulation of DFT [34, 35], once proper 'projections' are imposed, the semi-covariant derivatives and the semi-covariant curvatures all become completely covariant with respect to both diffeomorphisms and the local Lorentz symmetry, besides the $\mathbf{O}(D, D)$ T-duality. Within this setup, the maximal as well as half-maximal $D=10$ supersymmetric double field theories (SDFT) have been constructed to the full order in fermions $[36,37]$ where each term in the Lagrangians is completely covariant, see also the earlier formulation within Generalized Geometry [25, 26]. Berman and Lee then modified the semi-covariant formalism to be apt for the twisted generalized Lie derivative by introducing torsionful semi-covariant derivative connections [29]. However, it is fair to say that while these proposals all opened up novel aspects of the section condition and hence DFT itself, many ingredients were introduced $a d$ hoc by hand. Deeper systematic understanding has been desirable. 
It is the purpose of the present paper to propose such a geometric scheme to twist the maximal and the half-maximal supersymmetric double field theories of refs. [36, 37] and systematically derive the gauged supersymmetric double field theories. Essentially, as our main results, we show that the semi-covariant formalism itself can be twisted by the Scherk-Schwarz ansatz, without any arbitrariness. This enables us to address readily the supersymmetric completions. The twisted and hence gauged maximal as well as halfmaximal supersymmetric double field theories are then completely fixed by requiring the supersymmetry to be unbroken. Each term in the constructed Lagrangian is completely covariant with respect to the twisted diffeomorphisms, the $\mathbf{S p i n}(1,9) \times \mathbf{S p i n}(9,1)$ local Lorentz symmetries, and a subgroup of $\mathbf{O}(10,10)$ which preserves the structure constant. This complete covariance also ensures the internal coordinate independence.

The organization of the paper is as follows.

- In section 2, we revisit with care the semi-covariant formulation of the ungauged or untwisted double field theory [34, 35] and its supersymmetric extensions [36-38]. While reviewing them in a self-contained manner, we spell, for later use of twist, all the relevant exact formulas which hold without assuming any section condition. Such formulas have not been fully spelled elsewhere before.

- In section 3, we twist the double field theory with a simple Scherk-Schwarz ansatz. Following closely Grana and Marques [27], we analyze a set of consistency conditions for the closure of the twisted generalized Lie derivatives, which we call twistability conditions. We show that all the nice properties of the semi-covariant formalism, including its complete covariantizability, are still valid after the twist under the twistability conditions. In particular, we verify that the consistent definition of the twisted Ramond-Ramond cohomology requires one additional condition which is, after the diagonal gauge fixing of the twofold local Lorentz symmetries, consistent with the previous work by Geissbühler et al. [28].

- Section 4 contains our main results. Readers may want to have a glance of our final results therein, before reading the preparatory sections, 2 and 3. We present the maximal and the half-maximal supersymmetric gauged double field theories as the twists of the $\mathcal{N}=2$ and the $\mathcal{N}=1, D=10$ supersymmetric double field theories [36, 37]. In particular, we show the twisted maximal supersymmetric invariance calls for the same extra condition which the twisted R-R gauge symmetry demands as well.

- In section 5 we conclude with comments.

Although our supersymmetry analyses are explicit only up to the leading order, we argue in section 3 that the full order supersymmetric completions are guaranteed to work, as the higher order fermionic terms are immune to the "relaxation" of the section condition.

Conventions. Equations which hold due to the original section condition (1.1) and the alternative twistability conditions are denoted differently with the two distinct symbols, ' $\sim$ ' and 'E' respectively, besides the strict equality, '='. For the sake of simplicity 


\begin{tabular}{|c|c|c|}
\hline Name & Schematic formula & Debut equation \\
\hline Semi-covariant derivative & $\nabla_{A}=\partial_{A}+\Gamma_{A}$ & $(2.18)$ \\
Master semi-covariant derivative & $\mathcal{D}_{A}=\partial_{A}+\Gamma_{A}+\Phi_{A}+\bar{\Phi}_{A}$ & $(2.31)$ \\
R-R cohomology differential operators & $\mathcal{D}_{ \pm}$ & $(2.81)$ \\
U-derivative & $\dot{D}_{\dot{A}}=\dot{\partial}_{\dot{A}}+\Omega_{\dot{A}}$ & $(3.8)$ \\
U-twisted master semi-covariant derivative & $\dot{\mathcal{D}}_{\dot{A}}=\dot{D}_{\dot{A}}+\dot{\Gamma}_{\dot{A}}+\dot{\Phi}_{\dot{A}}+\dot{\bar{\Phi}}_{\dot{A}}$ & $(3.55)$ \\
\hline
\end{tabular}

Table 1. Various derivatives employed in the present paper.

\begin{tabular}{|c|c|c|}
\hline Index & Representation & Raising \& Lowering Indices \\
\hline$A, B, \cdots$ & Untwisted $\mathbf{O}(10,10)$ vector & $\mathcal{J}_{A B}=\left(\begin{array}{ll}0 & 1 \\
1 & 0\end{array}\right)$ \\
$\dot{A}, \dot{B}, \cdots$ & Twisted $\mathbf{O}(10,10)$ vector & $\dot{\mathcal{J}}_{\dot{A} \dot{B}}=\left(\begin{array}{ll}0 & 1 \\
1 & 0\end{array}\right)$ \\
$p, q, \cdots$ & $\operatorname{Spin}(1,9)$ vector & $\eta_{p q}=\operatorname{diag}(-++\cdots+)$ \\
$\bar{p}, \bar{q}, \cdots$ & $\operatorname{Spin}(9,1)$ vector & $\bar{\eta}_{\bar{p} \bar{q}}=\operatorname{diag}(+--\cdots-)$ \\
$\alpha, \beta, \cdots$ & $\operatorname{Spin}(1,9)$ spinor & $C_{+\alpha \beta},\left(\gamma^{p}\right)^{T}=C_{+} \gamma^{p} C_{+}^{-1}$ \\
$\bar{\alpha}, \bar{\beta}, \cdots$ & $\operatorname{Spin}(9,1)$ spinor & $\bar{C}_{+\bar{\alpha} \bar{\beta}},\left(\bar{\gamma}^{\bar{p}}\right)^{T}=\bar{C}_{+} \bar{\gamma}^{\bar{p}} \bar{C}_{+}^{-1}$ \\
\hline
\end{tabular}

Table 2. Index for each symmetry representation and the corresponding "metric" which raises or lowers its position. Only the capital $\mathbf{O}(10,10)$ indices are to be twisted. The ' + ' subscripts of the charge conjugation matrices indicate that they are chosen to be symmetric. The doubling of the local Lorentz symmetries, $\operatorname{Spin}(1,9) \rightarrow \operatorname{Spin}(1,9) \times \mathbf{S p i n}(9,1)$, is crucial to achieve the unification of IIA and IIB supergravities within the unique $\mathcal{N}=2, D=10$ untwisted SDFT [37].

we shall often adopt a matrix notation to suppress contracted indices, e.g. $\left(P \partial_{A} P\right)_{B} C=$ $P_{B}{ }^{E} \partial_{A} P_{E}^{C}$. Our index conventions follow [37] and are summarized in table 2. In table 1, we also list various derivatives which are explained and used throughout the paper.

\section{The semi-covariant formulation of ungauged DFT}

In this preparatory section, we revisit the semi-covariant formulation of ungauged or untwisted double field theory [34, 35] and its supersymmetric extensions [36-38]. Our goal is threefold: to review them in a self-contained manner, to locate the exact places where the original section condition is assumed, and to collect, for later use of twist, precise formulas which hold without assuming any section condition. Such formulas have not been fully spelled in the literature before. Every formula which holds up to the original section condition will be denoted by the symbol, ' $\sim$ ', rather than by the strict equality, ' $=$ '.

In particular, we pay attention to two strictly-different yet section-condition-equivalent semi-covariant four-index curvatures, namely $\mathcal{G}_{A B C D}$ and $S_{A B C D}$, and analyze their differences exactly without assuming the section condition. 


\subsection{Coordinate gauge symmetry, section condition and diffeomorphism}

- Doubled-yet-gauged spacetime. The spacetime is formally doubled, being $(D+D)$ dimensional. However, the doubled spacetime coordinates are gauged: the coordinate space is equipped with an equivalence relation,

$$
x^{A} \sim x^{A}+\phi \partial^{A} \varphi
$$

which is called 'coordinate gauge symmetry' $[8,10]$. In $(2.1), \phi$ and $\varphi$ are arbitrary functions in DFT.

Each equivalence class, or gauge orbit defined by the equivalence relation (2.1), represents a single physical point, and diffeomorphism symmetry means an invariance under arbitrary reparametrizations of the gauge orbits.

- Section condition: realization of the coordinate gauge symmetry. The equivalence relation (2.1) is realized in DFT by enforcing that, arbitrary functions and their arbitrary derivative descendants, denoted here collectively by $\Phi$, are invariant under the coordinate gauge symmetry shift $[8,10]$,

$$
\Phi(x+\Delta) \sim \Phi(x), \quad \Delta^{A}=\phi \partial^{A} \varphi .
$$

This invariance is equivalent, i.e. sufficient [8] and necessary [10] to the section condition,

$$
\partial_{A} \partial^{A} \sim 0 .
$$

Acting on arbitrary functions, $\Phi, \Phi^{\prime}$, and their products, the section condition leads to the weak constraint, $\partial_{A} \partial^{A} \Phi \sim 0$ as well as the strong constraint, $\partial_{A} \Phi \partial^{A} \Phi^{\prime} \sim 0$.

- Diffeomorphism. Diffeomorphism symmetry in DFT is generated by a generalized Lie derivative [1, 39, 40],

$$
\hat{\mathcal{L}}_{X} T_{A_{1} \cdots A_{n}}:=X^{B} \partial_{B} T_{A_{1} \cdots A_{n}}+\omega \partial_{B} X^{B} T_{A_{1} \cdots A_{n}}+\sum_{i=1}^{n}\left(\partial_{A_{i}} X_{B}-\partial_{B} X_{A_{i}}\right) T_{A_{1} \cdots A_{i-1}}{ }^{B} A_{i+1} \cdots A_{n},
$$

where $\omega$ denotes the weight of the field, $T_{A_{1} \cdots A_{n}}$. In particular, the generalized Lie derivative of the $\mathbf{O}(D, D)$ invariant metric is trivial,

$$
\hat{\mathcal{L}}_{X} \mathcal{J}_{A B}=0 .
$$

The commutator of the generalized Lie derivatives is closed by C-bracket [1, 41] up to the section condition,

$$
\begin{aligned}
{\left[\hat{\mathcal{L}}_{X}, \hat{\mathcal{L}}_{Y}\right] } & \sim \hat{\mathcal{L}}_{[X, Y]_{\mathrm{C}}}, \\
{[X, Y]_{\mathrm{C}}^{A} } & :=X^{B} \partial_{B} Y^{A}-Y^{B} \partial_{B} X^{A}+\frac{1}{2} Y^{B} \partial^{A} X_{B}-\frac{1}{2} X^{B} \partial^{A} Y_{B},
\end{aligned}
$$


since the following strict equality holds without resorting to the section condition [34],

$$
\begin{aligned}
\left(\left[\hat{\mathcal{L}}_{X}, \hat{\mathcal{L}}_{Y}\right]-\hat{\mathcal{L}}_{[X, Y]_{\mathrm{C}}}\right) T_{A_{1} \cdots A_{n}}= & \frac{1}{2}\left(X^{N} \partial^{M} Y_{N}-Y^{N} \partial^{M} X_{N}\right) \partial_{M} T_{A_{1} \cdots A_{n}} \\
& +\frac{1}{2} \omega\left(X^{N} \partial_{M} \partial^{M} Y_{N}-Y^{N} \partial_{M} \partial^{M} X_{N}\right) T_{A_{1} \cdots A_{n}} \\
& +\sum_{i=1}^{n}\left(\partial_{M} Y_{A_{i}} \partial^{M} X_{B}-\partial_{M} X_{A_{i}} \partial^{M} Y_{B}\right) T_{A_{1} \cdots A_{i-1}}{ }^{B}{ }_{A_{i+1} \cdots A_{n}}
\end{aligned}
$$

of which the right hand side clearly vanishes upon the section condition. We shall come back to this expression when we perform the twist.

\subsection{Dilaton, vielbeins and projectors}

- Dilaton and a pair of vielbeins. The geometric objects in DFT come from the closed string NS-NS sector and consist of a dilaton, $d$, and a pair of vielbeins, $V_{A p}, \bar{V}_{A \bar{p}}$. While the vielbeins are weightless, the dilaton gives rise to the $\mathbf{O}(D, D)$ invariant integral measure with weight one [41], after exponentiation,

$$
e^{-2 d} \text {. }
$$

The vielbeins satisfy the following four defining properties [35, 42] (see also [1, 40]):

$$
V_{A p} V_{q}^{A}=\eta_{p q}, \quad \bar{V}_{A \bar{p}} \bar{V}_{\bar{q}}^{A}=\bar{\eta}_{\bar{p} \bar{q}}, \quad V_{A p} \bar{V}_{\bar{q}}^{A}=0, \quad V_{A p} V_{B}^{p}+\bar{V}_{A \bar{p}} \bar{V}_{B}^{\bar{p}}=\mathcal{J}_{A B} .
$$

That is to say, they are normalized, orthogonal and complete. The vielbeins are $\mathbf{O}(D, D)$ vectors as their indices indicate. In fact, they are the only $\mathbf{O}(D, D)$ nonsinglet field variables even in the supersymmetric extensions of DFT [36, 37]. As a solution to (2.9), they can be parametrized in terms of ordinary zehnbeins and $B$ field, in various ways up to $\mathbf{O}(D, D)$ rotations and field redefinitions, e.g. [38, 43, 44].

Due to the defining properties of (2.9), arbitrary variations of the vielbeins meet

$$
\delta V_{A p}=\bar{P}_{A}^{B} \delta V_{B p}+V_{A}^{q} \delta V_{B[p} V_{q]}^{B}, \quad \delta \bar{V}_{A \bar{p}}=P_{A}^{B} \delta \bar{V}_{B \bar{p}}+\bar{V}_{A}^{\bar{q}} \delta \bar{V}_{B[\bar{p}} \bar{V}_{\bar{q}]}^{B} .
$$

- Projectors. The vielbeins generate a pair of symmetric, orthogonal and complete two-index projectors, ${ }^{1}$

$$
P_{A B}=P_{B A}=V_{A}^{p} V_{B p}, \quad \bar{P}_{A B}=\bar{P}_{B A}=\bar{V}_{A}^{\bar{p}} \bar{V}_{B \bar{p}},
$$

satisfying

$$
\begin{aligned}
& P_{A}{ }^{B} P_{B}{ }^{C}=P_{A}^{C}, \\
& \bar{P}_{A}^{B} \bar{P}_{B}^{C}=\bar{P}_{A}^{C}, \\
& P_{A}{ }^{B} \bar{P}_{B}^{C}=0, \\
& P_{A}{ }^{B}+\bar{P}_{A}^{B}=\delta_{A}{ }^{B} \text {, } \\
& \operatorname{tr}(P)=P_{A}{ }^{A}=D, \\
& \operatorname{tr}(\bar{P})=\bar{P}_{A}{ }^{A}=D .
\end{aligned}
$$

\footnotetext{
${ }^{1}$ The difference of the two projectors, , $P_{A B}-\bar{P}_{A B}=\mathcal{H}_{A B}$, corresponds to the "generalized metric" in [4], which can be also independently defined as a symmetric $\mathbf{O}(D, D)$ element, i.e. $\mathcal{H}_{A B}=\mathcal{H}_{B A}, \mathcal{H}_{A}{ }^{B} \mathcal{H}_{B}{ }^{C}=$ $\delta_{A}{ }^{C}$. However, in the 'full order' supersymmetric extensions of DFT [36, 37] where e.g. the 1.5 formalism works, it appears that the projectors are more fundamental than the "generalized metric".
} 
Further, the two-index projectors generate a pair of six-index projectors,

$$
\begin{aligned}
& \mathcal{P}_{A B C}{ }^{D E F}:=P_{A}{ }^{D} P_{[B}{ }^{[E} P_{C]}{ }^{F]}+\frac{2}{D-1} P_{A[B} P_{C]}{ }^{[E} P^{F] D}, \\
& \overline{\mathcal{P}}_{A B C}{ }^{D E F}:=\bar{P}_{A}^{D} \bar{P}_{[B}^{[E} \bar{P}_{C]}{ }^{F]}+\frac{2}{D-1} \bar{P}_{A[B} \bar{P}_{C]}\left[E \bar{P}^{F] D},\right.
\end{aligned}
$$

which satisfy the 'projection' property,

$$
\mathcal{P}_{A B C}{ }^{D E F} \mathcal{P}_{D E F}{ }^{G H I}=\mathcal{P}_{A B C}{ }^{G H I}, \quad \overline{\mathcal{P}}_{A B C}{ }^{D E F} \overline{\mathcal{P}}_{D E F}{ }^{G H I}=\overline{\mathcal{P}}_{A B C}{ }^{G H I},
$$

symmetric and traceless properties,

$$
\begin{array}{llll}
\mathcal{P}_{A B C D E F}=\mathcal{P}_{D E F A B C}, & \mathcal{P}_{A B C D E F}=\mathcal{P}_{A[B C] D[E F]}, & & P^{A B} \mathcal{P}_{A B C D E F}=0, \\
\overline{\mathcal{P}}_{A B C D E F}=\overline{\mathcal{P}}_{D E F A B C}, & \overline{\mathcal{P}}_{A B C D E F}=\overline{\mathcal{P}}_{A[B C] D[E F]}, & & \bar{P}^{A B} \overline{\mathcal{P}}_{A B C D E F}=0,
\end{array}
$$

as well as further properties like

$$
\begin{aligned}
\mathcal{P}_{[A B] C}{ }^{D E F} & =\mathcal{P}_{C A B}{ }^{[E F] D}, \quad \overline{\mathcal{P}}_{[A B] C}{ }^{D E F}=\overline{\mathcal{P}}_{C A B}{ }^{[E F] D}, \\
\frac{2}{3} \mathcal{P}_{[A B] C}{ }^{D E F}+\frac{1}{3} \mathcal{P}_{C A B}{ }^{D E F} & =\mathcal{P}_{[A B C]}{ }^{D E F}=\mathcal{P}_{[A B C]}^{[D E F]}, \\
\frac{2}{3} \overline{\mathcal{P}}_{[A B] C}{ }^{D E F}+\frac{1}{3} \overline{\mathcal{P}}_{C A B}{ }^{D E F} & =\overline{\mathcal{P}}_{[A B C]}^{D E F}=\overline{\mathcal{P}}_{[A B C]}^{[D E F]} .
\end{aligned}
$$

In addition to the six-index projection operators (2.13), we also set for later use,

$$
\begin{gathered}
\mathcal{P}_{C A B}^{\prime}{ }^{F D E}:=\bar{P}_{C}{ }^{F} P_{[A}{ }^{[D} P_{B]}^{E]}+\frac{2}{D-1} P_{C[A} P_{B]}\left[D \bar{P}^{E] F},\right. \\
\overline{\mathcal{P}}_{C A B}^{\prime}{ }^{F D E}:=P_{C}{ }^{F} \bar{P}_{[A}\left[D \bar{P}_{B]}^{E]}+\frac{2}{D-1} \bar{P}_{C[A} \bar{P}_{B]}\left[D P^{E] F} .\right.\right.
\end{gathered}
$$

\subsection{Semi-covariant derivatives, curvatures and their complete covariantiza- tions}

- Semi-covariant derivative and the torsionless connection. The semi-covariant derivative is defined by $[34,35]$

$$
\nabla_{C} T_{A_{1} A_{2} \cdots A_{n}}:=\partial_{C} T_{A_{1} A_{2} \cdots A_{n}}-\omega_{T} \Gamma^{B}{ }_{B C} T_{A_{1} A_{2} \cdots A_{n}}+\sum_{i=1}^{n} \Gamma_{C A_{i}}{ }^{B} T_{A_{1} \cdots A_{i-1} B A_{i+1} \cdots A_{n}} .
$$

It satisfies the Leibniz rule and is compatible with the $\mathbf{O}(D, D)$ invariant constant metric,

$$
\nabla_{A} \mathcal{J}_{B C}=0 .
$$

We choose the connection to be the torsionless one from ref. [35]:2

$$
\begin{aligned}
\Gamma_{C A B}= & 2\left(P \partial_{C} P \bar{P}\right)_{[A B]}+2\left(\bar{P}_{[A}^{D} \bar{P}_{B]}^{E}-P_{[A}^{D} P_{B]}^{E}\right) \partial_{D} P_{E C} \\
& -\frac{4}{D-1}\left(\bar{P}_{C[A} \bar{P}_{B]}^{D}+P_{C[A} P_{B]}^{D}\right)\left(\partial_{D} d+\left(P \partial^{E} P \bar{P}\right)_{[E D]}\right),
\end{aligned}
$$

\footnotetext{
${ }^{2}$ The connection (2.20) of [35] was reviewed further from a slightly different angle in [45].
} 
which is a unique solution to the following five constraints [35]:

$$
\begin{aligned}
\nabla_{A} P_{B C} & =0, \quad \nabla_{A} \bar{P}_{B C}=0, \\
\nabla_{A} d & =-\frac{1}{2} e^{2 d} \nabla_{A}\left(e^{-2 d}\right)=\partial_{A} d+\frac{1}{2} \Gamma_{B A}^{B}=0, \\
\Gamma_{A B C}+\Gamma_{A C B} & =0, \\
\Gamma_{A B C}+\Gamma_{B C A}+\Gamma_{C A B} & =0, \\
\mathcal{P}_{A B C}{ }^{D E F} \Gamma_{D E F} & =0, \quad \overline{\mathcal{P}}_{A B C}{ }^{D E F} \Gamma_{D E F}=0 .
\end{aligned}
$$

The first two relations, $(2.21),(2.22)$, are the compatibility conditions with the dilaton and the projectors, i.e. the whole NS-NS sector. The third constraint (2.23) is the compatibility condition with the $\mathbf{O}(D, D)$ invariant constant metric, (2.19). The next cyclic property, (2.24), makes the semi-covariant derivative compatible with the generalized Lie derivative as well as with the C-bracket,

$$
\hat{\mathcal{L}}_{X}(\partial)=\hat{\mathcal{L}}_{X}(\nabla), \quad[X, Y]_{\mathrm{C}}(\partial)=[X, Y]_{\mathrm{C}}(\nabla) .
$$

The last formulae (2.25) are projection conditions which ensure the uniqueness.

While the torsionless connection satisfies all the five constraints, (2.21)-(2.25) and thus uniquely determined, a generic torsionful connection meets only the first three conditions, (2.21), (2.22), (2.23), and decomposes into the torsionless connection and torsions $[25,36]$,

$$
\Gamma_{C A B}+\Delta_{C p q} V_{A}^{p} V_{B}^{q}+\bar{\Delta}_{C \bar{p} \bar{q}} \bar{V}_{A}{ }^{\bar{p}} \bar{V}_{B}{ }^{\bar{q}} .
$$

In order to maintain (2.22), the torsions must satisfy

$$
\Delta_{A p q}=\Delta_{A[p q]}, \quad \Delta_{A p q} V^{A p}=0, \quad \bar{\Delta}_{A \bar{p} \bar{q}}=\bar{\Delta}_{A[\bar{p} \bar{q}]}, \quad \bar{\Delta}_{A \bar{p} \bar{q}} \bar{V}^{A \bar{p}}=0 .
$$

In the full order supersymmetric extensions of DFT [36, 37], they are given by quadratic fermions.

It is worth while to note

$$
P_{I}^{A} \bar{P}_{J}^{B} \Gamma_{C A B}=\left(P \partial_{C} P \bar{P}\right)_{I J},
$$

such that

$$
\Gamma_{p \bar{q}}^{C} \partial_{C}=V_{p}^{A} \bar{V}_{\bar{q}}^{B} \Gamma_{A B}^{C} \partial_{C} \sim 0 .
$$

- Spin connections and semi-covariant master derivative. The master semi-covariant derivative [42],

$$
\mathcal{D}_{A}:=\nabla_{A}+\Phi_{A}+\bar{\Phi}_{A}=\partial_{A}+\Gamma_{A}+\Phi_{A}+\bar{\Phi}_{A},
$$

generalizes the semi-covariant derivative, $\nabla_{A}(2.18)$, to include the spin connections, $\Phi_{A}$ and $\bar{\Phi}_{A}$, for the two local Lorentz groups, $\operatorname{Spin}(1, D-1)_{L}$ and $\operatorname{Spin}(D-1,1)_{R}$ respectively. By definition, it is compatible with the vielbeins,

$$
\begin{aligned}
& \mathcal{D}_{A} V_{B p}=\partial_{A} V_{B p}+\Gamma_{A B}{ }^{C} V_{C p}+\Phi_{A p}{ }^{q} V_{B q}=0, \\
& \mathcal{D}_{A} \bar{V}_{A \bar{p}}=\partial_{A} \bar{V}_{B \bar{p}}+\Gamma_{A B}{ }^{C} \bar{V}_{C \bar{p}}+\bar{\Phi}_{A \bar{p}} \bar{q} \bar{V}_{B \bar{q}}=0,
\end{aligned}
$$


and, from (2.22), also with the dilaton,

$$
\mathcal{D}_{A} d=\nabla_{A} d=0
$$

The connections are then related to each other by

$$
\Phi_{A p q}=\Phi_{A[p q]}=V_{p}^{B} \nabla_{A} V_{B q}, \quad \bar{\Phi}_{A \bar{p} \bar{q}}=\bar{\Phi}_{A[\bar{p} \bar{q}]}=\bar{V}_{\bar{p}}^{B} \nabla_{A} \bar{V}_{B \bar{q}}
$$

and

$$
\begin{aligned}
\Gamma_{A B C} & =V_{B}^{p}\left(\partial_{A} V_{C p}+\Phi_{A p}^{q} V_{C q}\right)+\bar{V}_{B}^{\bar{p}}\left(\partial_{A} \bar{V}_{C \bar{p}}+\bar{\Phi}_{A \bar{p}}{ }^{\bar{q}} \bar{V}_{C \bar{q}}\right) \\
& =V_{B}{ }^{p} \partial_{A} V_{C p}+\bar{V}_{B}{ }^{\bar{p}} \partial_{A} \bar{V}_{C \bar{p}}+\Phi_{A B C}+\bar{\Phi}_{A B C} .
\end{aligned}
$$

Consequently, their generic infinitesimal variations satisfy

$$
\delta \Phi_{A p q}=\mathcal{D}_{A}\left(V_{p}^{B} \delta V_{B q}\right)+V_{p}^{B} V_{q}^{C} \delta \Gamma_{A B C}, \quad \delta \bar{\Phi}_{A \bar{p} \bar{q}}=\mathcal{D}_{A}\left(\bar{V}^{B}{ }_{\bar{p}} \delta \bar{V}_{B \bar{q}}\right)+\bar{V}^{B}{ }_{\bar{p}} \bar{V}^{C}{ }_{\bar{q}} \delta \Gamma_{A B C} .
$$

The master semi-covariant derivative is also compatible with all the constant metrics and the gamma matrices in table 2 ,

$$
\mathcal{D}_{A} \mathcal{J}_{B C}=0, \quad \mathcal{D}_{A} \eta_{p q}=0, \quad \mathcal{D}_{A} \bar{\eta}_{\bar{p} \bar{q}}=0, \quad \mathcal{D}_{A}\left(\gamma^{p}\right)^{\alpha}{ }_{\beta}=0, \quad \mathcal{D}_{A}\left(\bar{\gamma}^{\bar{p}}\right)^{\bar{\alpha}}{ }_{\bar{\beta}}=0
$$

The well known relation between the spinorial and the vectorial representations of the spin connections follows

$$
\Phi_{A}{ }_{\beta}=\frac{1}{4} \Phi_{A p q}\left(\gamma^{p q}\right)^{\alpha}, \quad \bar{\Phi}_{A} \bar{\alpha}_{\bar{\beta}}=\frac{1}{4} \bar{\Phi}_{A \bar{p} \bar{q}}\left(\bar{\gamma}^{\bar{p} \bar{q}}\right)^{\bar{\alpha}}{ }_{\bar{\beta}} .
$$

- Semi-covariant four-index curvatures. The usual "field strengths" of the three connections,

$$
\begin{aligned}
R_{C D A B} & =\partial_{A} \Gamma_{B C D}-\partial_{B} \Gamma_{A C D}+\Gamma_{A C}{ }^{E} \Gamma_{B E D}-\Gamma_{B C}{ }^{E} \Gamma_{A E D}, \\
F_{A B p q} & =\partial_{A} \Phi_{B p q}-\partial_{B} \Phi_{A p q}+\Phi_{A p r} \Phi_{B}{ }^{r}{ }_{q}-\Phi_{B p r} \Phi_{A}^{r}{ }_{q} \\
\bar{F}_{A B \bar{p} \bar{q}} & =\partial_{A} \bar{\Phi}_{B \bar{p} \bar{q}}-\partial_{B} \bar{\Phi}_{A \bar{p} \bar{q}}+\bar{\Phi}_{A \bar{p} \bar{r}} \bar{\Phi}_{B}{ }^{\bar{r}}{ }_{\bar{q}}-\bar{\Phi}_{B \bar{p} \bar{r}} \bar{\Phi}_{A}{ }^{\bar{r}} \bar{q}
\end{aligned}
$$

are, from $\left[\mathcal{D}_{A}, \mathcal{D}_{B}\right] V_{C p}=0$ and $\left[\mathcal{D}_{A}, \mathcal{D}_{B}\right] \bar{V}_{C \bar{p}}=0$, related to each other by

$$
R_{A B C D}=F_{C D p q} V_{A}^{p} V_{B}^{q}+\bar{F}_{C D \bar{p} \bar{q}} \bar{V}_{A}^{\bar{p}} \bar{V}_{B}^{\bar{q}}=F_{C D A B}+\bar{F}_{C D A B} .
$$

This implies

$$
R_{A B C D}=R_{[A B][C D]}, \quad R_{p \bar{q} C D}=V_{p}^{A} \bar{V}_{\bar{q}}^{B} R_{A B C D}=0 .
$$

Following [46], replacing the ordinary or the naked derivatives in (2.39) by the semicovariant derivatives we define

$$
\begin{aligned}
\mathcal{F}_{A B p q} & :=\nabla_{A} \Phi_{B p q}-\nabla_{B} \Phi_{A p q}+\Phi_{A p}{ }^{r} \Phi_{B r q}-\Phi_{B p}{ }^{r} \Phi_{A r q}, \\
\overline{\mathcal{F}}_{A B \bar{p} \bar{q}} & :=\nabla_{A} \bar{\Phi}_{B \bar{p} \bar{q}}-\nabla_{B} \bar{\Phi}_{A \bar{p} \bar{q}}+\bar{\Phi}_{A \bar{p}}{ }^{\bar{r}} \bar{\Phi}_{B \bar{r} \bar{q}}-\bar{\Phi}_{B \bar{p}} \bar{\Phi}_{A \bar{r} \bar{q}},
\end{aligned}
$$


which are, with the torsion-free condition (2.24), related to (2.39) by

$$
\mathcal{F}_{A B p q}=F_{A B p q}-\Gamma_{A B}^{C} \Phi_{C p q}, \quad \overline{\mathcal{F}}_{A B \bar{p} \bar{q}}=\bar{F}_{A B \bar{p} \bar{q}}-\Gamma^{C}{ }_{A B} \bar{\Phi}_{C \bar{p} \bar{q}},
$$

and appear in the commutators of the master semi-covariant derivatives,

$$
\left[\mathcal{D}_{A}, \mathcal{D}_{B}\right] T_{p}=\mathcal{F}_{A B p q} T^{q}-\Gamma^{C}{ }_{A B} \partial_{C} T_{p}, \quad\left[\mathcal{D}_{A}, \mathcal{D}_{B}\right] T_{\bar{p}}=\overline{\mathcal{F}}_{A B \bar{p} \bar{q}} T^{\bar{q}}-\Gamma^{C}{ }_{A B} \partial_{C} T_{\bar{p}}
$$

Further, they can be rewritten in terms of the master semi-covariant derivatives, then to carry some opposite signs in comparison to (2.42),

$$
\begin{aligned}
\mathcal{F}_{A B p q} & =\mathcal{D}_{A} \Phi_{B p q}-\mathcal{D}_{B} \Phi_{A p q}-\Phi_{A p}{ }^{r} \Phi_{B r q}+\Phi_{B p}{ }^{r} \Phi_{A r q} \\
\overline{\mathcal{F}}_{A B \bar{p} \bar{q}} & =\mathcal{D}_{A} \bar{\Phi}_{B \bar{p} \bar{q}}-\mathcal{D}_{B} \bar{\Phi}_{A \bar{p} \bar{q}}-\bar{\Phi}_{A \bar{p}}{ }^{r} \bar{\Phi}_{B \bar{r} \bar{q}}+\bar{\Phi}_{B \bar{p}}{ }^{r} \bar{\Phi}_{A \bar{r} \bar{q}}
\end{aligned}
$$

Hence, contracted with the vielbeins - which are compatible with $\mathcal{D}_{A}$ but not with $\nabla_{A}$ - we may write

$$
\begin{aligned}
& \mathcal{F}_{A B C D}=\mathcal{F}_{A B p q} V_{C}^{p} V_{D}^{q}=\nabla_{A} \Phi_{B C D}-\nabla_{B} \Phi_{A C D}-\Phi_{A C}{ }^{E} \Phi_{B E D}+\Phi_{B C}{ }^{E} \Phi_{A E D} \\
& \overline{\mathcal{F}}_{A B C D}=\overline{\mathcal{F}}_{A B \bar{p} \bar{q}} \bar{V}_{C}^{\bar{p}} \bar{V}_{D}^{\bar{q}}=\nabla_{A} \bar{\Phi}_{B C D}-\nabla_{B} \bar{\Phi}_{A C D}-\bar{\Phi}_{A C}{ }^{E} \bar{\Phi}_{B E D}+\bar{\Phi}_{B C}{ }^{E} \bar{\Phi}_{A E D} .
\end{aligned}
$$

Now we are ready to define two kinds of semi-covariant four-index curvatures:

- Semi-covariant four-index curvature of the spin connections, cf. [28],

$$
\mathcal{G}_{A B C D}:=\frac{1}{2}\left[(\mathcal{F}+\overline{\mathcal{F}})_{A B C D}+(\mathcal{F}+\overline{\mathcal{F}})_{C D A B}+(\Phi+\bar{\Phi})^{E}{ }_{A B}(\Phi+\bar{\Phi})_{E C D}\right] .
$$

- Semi-covariant Riemann curvature of the diffeomorphic connection [34, 35],

$$
S_{A B C D}:=\frac{1}{2}\left(R_{A B C D}+R_{C D A B}-\Gamma_{A B}^{E} \Gamma_{E C D}\right) .
$$

These two four-index curvatures are closely related to each other,

$$
\begin{aligned}
\mathcal{G}_{A B C D} & =S_{A B C D}+\frac{1}{2}(\Gamma-\Phi-\bar{\Phi})_{E A B}(\Gamma-\Phi-\bar{\Phi})^{E} C D \\
& =S_{A B C D}+\frac{1}{2}\left(V_{A}^{p} \partial_{E} V_{B p}+\bar{V}_{A}^{\bar{p}} \partial_{E} \bar{V}_{B \bar{p}}\right)\left(V_{C}^{q} \partial^{E} V_{D q}+\bar{V}_{C}^{\bar{q}} \partial^{E} \bar{V}_{D \bar{q}}\right),
\end{aligned}
$$

such that upon the section condition we have

$$
\mathcal{G}_{A B C D} \sim S_{A B C D}
$$

As a bonus, this implies that, up to the section condition $\mathcal{G}_{A B C D}$ is local Lorentz invariant as $S_{A B C D}$ is so. Note that while $F_{A B p q}$ and $\bar{F}_{A B \bar{p} \bar{q}}$ are local Lorentz covariant, $\mathcal{F}_{A B p q}$ and $\overline{\mathcal{F}}_{A B \bar{p} \bar{q}}$ are not.

A notable difference between $\mathcal{G}_{A B C D}$ and $S_{A B C D}$ is that while the latter can be expressed in terms of the dilaton and the projectors, the former cannot be defined thoroughly by them: it requires the vielbeins. In the following section, we shall see 
that it is $\mathcal{G}_{A B C D}$ rather than $S_{A B C D}$ that survives to serve as the semi-covariant curvature after the twist.

It is worth while to note that, in the expressions of $\Phi_{A p q}, \bar{\Phi}_{A \bar{p} \bar{q}}(2.34), \mathcal{F}_{A B p q}$, $\overline{\mathcal{F}}_{A B \bar{p} \bar{q}}(2.42)$ and $\mathcal{G}_{A B C D}(2.47)$, the ordinary naked derivative and the $\Gamma$-connection are completely 'confined' into the semi-covariant derivative. On the other hand, it is not the case with $R_{A B C D}, F_{A B p q}, \bar{F}_{A B \bar{p} \bar{q}}$ and $S_{A B C D}$.

A crucial defining property of the semi-covariant Riemann curvature is that, under arbitrary transformation of the connection, it transforms as

$$
\delta S_{A B C D}=\nabla_{[A} \delta \Gamma_{B] C D}+\nabla_{[C} \delta \Gamma_{D] A B}-\frac{3}{2} \Gamma_{[A B E]} \delta \Gamma^{E}{ }_{C D}-\frac{3}{2} \Gamma_{[C D E]} \delta \Gamma_{A B}^{E} .
$$

Surely for the torsion-free connection (2.20), the last two terms are absent and only the first two total derivative terms remain,

$$
\delta S_{A B C D}=\nabla_{[A} \delta \Gamma_{B] C D}+\nabla_{[C} \delta \Gamma_{D] A B}
$$

Yet, in the full order supersymmetric extensions of DFT [36, 37], the connection includes bi-fermionic torsions and the above general relation (2.51) enables the ' 1.5 formalism' to work.

Without necessity of the section condition, $S_{A B C D}$ satisfies [34],

$$
S_{A B C D}=S_{[A B][C D]}=S_{C D A B}
$$

and, especially for the torsionless connection, a Bianchi identity, ${ }^{3}$

$$
S_{A[B C D]}=0 .
$$

Further, for the torsionless connection (2.20), one can show by a brute-force method, ${ }^{4}$

$$
\begin{aligned}
\left(P^{A B} P^{C D}+\bar{P}^{A B} \bar{P}^{C D}\right) S_{A C B D}= & 4 \partial_{A} \partial^{A} d-4 \partial_{A} d \partial^{A} d+\frac{1}{2} \partial_{A} P_{C D} \partial^{A} P^{C D} \sim 0, \\
P_{I}^{A} P_{J}^{B} \bar{P}_{K}^{C} \bar{P}_{L}^{D} S_{A B C D}= & \frac{1}{2}\left(P \partial_{A} P \bar{P}\right)_{I L}\left(P \partial^{A} P \bar{P}\right)_{J K} \\
& -\frac{1}{2}\left(P \partial_{A} P \bar{P}\right)_{I K}\left(P \partial^{A} P \bar{P}\right)_{J L} \sim 0, \\
P_{I}^{A} \bar{P}_{J}^{B} P_{K}^{C} \bar{P}_{L}^{D} S_{A B C D}= & -\frac{1}{2}\left(P \partial_{A} P \bar{P}\right)_{I J}\left(P \partial^{A} P \bar{P}\right)_{K L} \sim 0, \\
P_{I}{ }^{A} \bar{P}_{J}^{B}(P-\bar{P})^{C D} S_{A C B D}= & -\frac{1}{2} P_{I}^{A} \bar{P}_{J}^{B} \partial_{C} \partial^{C} P_{A B}+\left(P \partial_{C} P \bar{P}\right)_{I J} \partial^{C} d \sim 0,
\end{aligned}
$$

of which the right hand sides all vanish upon the section condition, $\partial_{A} \partial^{A} \sim 0$.

It follows, from (2.50), that identical relations hold for $\mathcal{G}_{A B C D}$, either by the strict equality or up to the section condition, for example,

$$
\mathcal{G}_{A B C D}=\mathcal{G}_{[A B][C D]}=\mathcal{G}_{C D A B}, \quad \mathcal{G}_{A[B C D]} \sim 0 .
$$

\footnotetext{
${ }^{3}$ See eq. (2.46) of [34] for a simple proof of the Bianchi identity.

${ }^{4}$ To obtain (2.55), we have used the computer algebra, Cadabra [47, 48].
} 
- Complete covariantizations. The ordinary derivative of a covariant tensor is no longer covariant under diffeomorphisms. The difference between its actual diffeomorphic transformation and the generalized Lie derivative reads precisely,

$$
\begin{aligned}
\left(\delta_{X}-\hat{\mathcal{L}}_{X}\right) \partial_{C} T_{A_{1} \cdots A_{n}}= & {\left[\partial_{C}, \hat{\mathcal{L}}_{X}\right] T_{A_{1} \cdots A_{n}} } \\
= & \partial^{B} X_{C} \partial_{B} T_{A_{1} \cdots A_{n}}+\omega_{T} \partial_{C} \partial_{B} X^{B} T_{A_{1} \cdots A_{n}} \\
& +\sum_{i=1}^{n} 2 \partial_{C} \partial_{\left[A_{i}\right.} X_{B]} T_{A_{1} \cdots A_{i-1}}{ }^{B}{ }_{A_{i+1} \cdots A_{n}}
\end{aligned}
$$

Especially for the connection we have

$$
\begin{aligned}
\left(\delta_{X}-\hat{\mathcal{L}}_{X}\right) \Gamma_{C A B}= & 2\left[(\mathcal{P}+\overline{\mathcal{P}})_{C A B}{ }^{F D E}-\delta_{C}^{F} \delta_{A}^{D} \delta_{B}^{E}\right] \partial_{F} \partial_{[D} X_{E]} \\
& +2\left(\mathcal{P}^{\prime}-\overline{\mathcal{P}}^{\prime}\right)_{C A B}{ }^{F D E} \partial^{G} P_{F D} \partial_{G} X_{E}+2 P_{[A}{ }^{D} \bar{P}_{B]}{ }^{E} \partial^{G} P_{D E} \partial_{G} X_{C} \\
& +\frac{2}{D-1}\left(P_{C[A} P_{B]}^{E}+\bar{P}_{C[A} \bar{P}_{B]}^{E}\right)\left(\partial_{G} \partial^{G} X_{E}-2 \partial^{G} d \partial_{G} X_{E}\right) .
\end{aligned}
$$

It follows that

$$
\left(\delta_{X}-\hat{\mathcal{L}}_{X}\right) \Gamma_{A B}^{A}=-2 \partial^{C} d \partial_{C} X_{B}+\partial_{B} \partial_{C} X^{C}=-2\left(\delta_{X}-\hat{\mathcal{L}}_{X}\right) \partial_{B} d .
$$

Further, using

$$
\begin{aligned}
{\left[\nabla_{C}, \hat{\mathcal{L}}_{X}\right] T_{A_{1} \cdots A_{n}}=} & \partial^{B} X_{C} \partial_{B} T_{A_{1} \cdots A_{n}}+\omega_{T}\left(\partial_{C} \partial_{B} X^{B}+\hat{\mathcal{L}}_{X} \Gamma^{B}{ }_{B C}\right) T_{A_{1} \cdots A_{n}} \\
& +\sum_{i=1}^{n}\left(2 \partial_{C} \partial_{\left[A_{i}\right.} X_{B]}-\hat{\mathcal{L}}_{X} \Gamma_{C A_{i} B}\right) T_{A_{1} \cdots A_{i-1}}{ }^{B}{ }_{A_{i+1} \cdots A_{n}}
\end{aligned}
$$

we may obtain an exact expression of the diffeomorphic anomaly of the semi-covariant derivative,

$$
\begin{aligned}
\left(\delta_{X}-\hat{\mathcal{L}}_{X}\right)\left(\nabla_{C} T_{A_{1} \cdots A_{n}}\right)= & \partial^{B} X_{C} \partial_{B} T_{A_{1} \cdots A_{n}}+\omega_{T}\left[\partial_{C} \partial_{B} X^{B}-\left(\delta_{X}-\hat{\mathcal{L}}_{X}\right) \Gamma^{B}{ }_{B C}\right] T_{A_{1} \cdots A_{n}} \\
& +\sum_{i=1}^{n}\left[2 \partial_{C} \partial_{\left[A_{i}\right.} X_{B]}+\left(\delta_{X}-\hat{\mathcal{L}}_{X}\right) \Gamma_{C A_{i} B}\right] T_{A_{1} \cdots A_{i-1}}{ }^{B}{ }_{A_{i+1} \cdots A_{n}}
\end{aligned}
$$

into which (2.58) can be readily substituted.

Lastly for the semi-covariant Riemannian curvature of the torsionless connection, from

$$
\hat{\mathcal{L}}_{X} S_{A B C D}=\nabla_{[A} \hat{\mathcal{L}}_{X} \Gamma_{B] C D}-2 \nabla_{[A} \partial_{B]} \partial_{[C} X_{D]}-\partial_{E} X_{[A} \partial^{E} \Gamma_{B] C D}+[(A, B) \leftrightarrow(C, D)],
$$

we get an exact formula,

$$
\begin{aligned}
\left(\delta_{X}-\hat{\mathcal{L}}_{X}\right) S_{A B C D}= & \nabla_{[A}\left(\delta_{X}-\hat{\mathcal{L}}_{X}\right) \Gamma_{B] C D}+2 \nabla_{[A} \partial_{B]} \partial_{[C} X_{D]}+\partial_{E} X_{[A} \partial^{E} \Gamma_{B] C D} \\
& +[(A, B) \leftrightarrow(C, D)] .
\end{aligned}
$$


Now, we consider imposing the section condition, $\partial_{A} \partial^{A} \sim 0$. Clearly, from (2.58), (2.61), (2.63) and (2.49), we note

$$
\begin{aligned}
\left(\delta_{X}-\hat{\mathcal{L}}_{X}\right) \Gamma_{C A B} & \sim 2\left[(\mathcal{P}+\overline{\mathcal{P}})_{C A B}{ }^{F D E}-\delta_{C}^{F} \delta_{A}^{D} \delta_{B}^{E}\right] \partial_{F} \partial_{[D} X_{E]}, \\
\left(\delta_{X}-\hat{\mathcal{L}}_{X}\right) \nabla_{C} T_{A_{1} \cdots A_{n}} & \sim \sum_{i=1}^{n} 2(\mathcal{P}+\overline{\mathcal{P}})_{C A_{i}}{ }^{B D E F} \partial_{D} \partial_{E} X_{F} T_{A_{1} \cdots A_{i-1} B A_{i+1} \cdots A_{n}},
\end{aligned}
$$

and for the four-index curvatures,

$$
\begin{aligned}
\left(\delta_{X}-\hat{\mathcal{L}}_{X}\right) \mathcal{G}_{A B C D} & \sim\left(\delta_{X}-\hat{\mathcal{L}}_{X}\right) S_{A B C D} \\
& \sim 2 \nabla_{[A}\left((\mathcal{P}+\overline{\mathcal{P}})_{B][C D]}{ }^{E F G} \partial_{E} \partial_{F} X_{G}\right)+[(A, B) \leftrightarrow(C, D)] .
\end{aligned}
$$

Thus, upon the section condition, it is the six-index projection operators that dictate the anomalies in the diffeomorphic transformations of the semi-covariant derivative and the semi-covariant curvatures. This also explains or motivates the naming, 'semicovariant': we say a tensor is semi-covariant if its diffeomorphic anomaly, if any, is governed by the six-index projectors.

The anomalous terms can be easily projected out through appropriate contractions with the two-index projectors. In this manner, the completely covariant derivatives are given by

$P_{C}{ }^{D} \bar{P}_{A_{1}}{ }^{B_{1}} \cdots \bar{P}_{A_{n}}^{B_{n}} \nabla_{D} T_{B_{1} \cdots B_{n}}, \quad \bar{P}_{C}^{D} P_{A_{1}}^{B_{1}} \cdots P_{A_{n}}{ }^{B_{n}} \nabla_{D} T_{B_{1} \cdots B_{n}}$, $P^{A B} \bar{P}_{C_{1}}{ }^{D_{1}} \ldots \bar{P}_{C_{n}}{ }^{D_{n}} \nabla_{A} T_{B D_{1} \cdots D_{n}}, \quad \bar{P}^{A B} P_{C_{1}}{ }^{D_{1}} \cdots P_{C_{n}}{ }^{D_{n}} \nabla_{A} T_{B D_{1} \cdots D_{n}} \quad$ (divergences), $P^{A B} \bar{P}_{C_{1}}{ }^{D_{1}} \ldots \bar{P}_{C_{n}}{ }^{D_{n}} \nabla_{A} \nabla_{B} T_{D_{1} \cdots D_{n}}, \quad \bar{P}^{A B} P_{C_{1}}{ }^{D_{1}} \ldots P_{C_{n}}{ }^{D_{n}} \nabla_{A} \nabla_{B} T_{D_{1} \cdots D_{n}} \quad$ (Laplacians).

These can be also freely pull-backed by the vielbeins to take the form:

$$
\mathcal{D}_{p} T_{\bar{q}_{1} \cdots \bar{q}_{n}}, \quad \mathcal{D}_{\bar{p}} T_{q_{1} \cdots q_{n}}, \quad \mathcal{D}_{p} T_{\bar{q}_{1} \cdots \bar{q}_{n}}^{p}, \quad \mathcal{D}_{\bar{p}} T_{q_{1} \cdots q_{n}}^{\bar{p}}, \quad \mathcal{D}_{p} \mathcal{D}^{p} T_{\bar{q}_{1} \cdots \bar{q}_{n}}, \quad \mathcal{D}_{\bar{p}} \mathcal{D}^{\bar{p}} T_{q_{1} \cdots q_{n}} .
$$

Similarly we obtain completely covariant two-index as well as zero-index curvatures from the semi-covariant four-index curvatures.

- Completely covariant "Ricci" curvatures, ${ }^{5}$

$$
\begin{aligned}
& \mathcal{G}_{p r \bar{q}}{ }^{r}=\frac{1}{2} \mathcal{F}_{\bar{q} r p}{ }^{r}=S_{p r \bar{q}}{ }^{r}+\frac{1}{2} P^{A B} \partial_{E} V_{A p} \partial^{E} \bar{V}_{B \bar{q}}, \\
& \mathcal{G}_{p \bar{r} \bar{q}}{ }^{\bar{r}}=\frac{1}{2} \overline{\mathcal{F}}_{p \bar{r} \bar{q}}{ }^{\bar{r}}=S_{p \bar{r} \bar{q}} \bar{r}+\frac{1}{2} \bar{P}^{A B} \partial_{E} V_{A p} \partial^{E} \bar{V}_{B \bar{q}},
\end{aligned}
$$

whose sum gives [38]

$$
\mathcal{G}_{p \bar{q}}=S_{p \bar{q}}+\frac{1}{2} \partial_{A} V_{B p} \partial^{A} \bar{V}^{B}{ }_{\bar{q}} \sim S_{p \bar{q}}
$$

- Completely covariant scalar curvatures,

$$
\begin{aligned}
& \mathcal{G}_{p q}^{p q}=\mathcal{F}_{p q}^{p q}+\frac{1}{2} \Phi_{E p q} \Phi^{E p q}=P^{A C} P^{B D} S_{A B C D}+\frac{1}{2} P^{A B} \partial_{E} V_{A p} \partial^{E} V_{B}^{p} \sim S_{p q}^{p q} \\
& \mathcal{G}_{\bar{p} \bar{q}}^{\bar{p} \bar{q}}=\overline{\mathcal{F}}_{\bar{p} \bar{q}}^{\bar{p} \bar{q}}+\frac{1}{2} \bar{\Phi}_{E \bar{p} \bar{q}} \bar{\Phi}^{E \bar{p} \bar{q}}=\bar{P}^{A C} \bar{P}^{B D} S_{A B C D}+\frac{1}{2} \bar{P}^{A B} \partial_{E} \bar{V}_{A \bar{p}} \partial^{E} \bar{V}_{B} \bar{p} \sim S_{\bar{p} \bar{q}}^{\bar{p} \bar{q}}
\end{aligned}
$$

\footnotetext{
${ }^{5}$ The expression (2.69) is for the torsionless connection. For torsionful extension, see [36, 37] and especially (A.71) of [38].
} 
In fact, the two "Ricci" curvatures agree to each other upon the section condition: from the identity (2.55), their difference reads exactly,

$$
\mathcal{G}_{p r \bar{q}}{ }^{r}-\mathcal{G}_{p \bar{r} \bar{q}}^{\bar{r}}=V_{p}^{A} \bar{V}^{B}{ }_{\bar{q}}\left(\partial_{E} P_{A B} \partial^{E} d-\frac{1}{2} \partial_{E} \partial^{E} P_{A B}\right)+\frac{1}{2}(P-\bar{P})^{A B} \partial_{E} V_{A p} \partial^{E} \bar{V}_{B \bar{q}},
$$

and hence,

$$
\mathcal{G}_{p r \bar{q}}{ }^{r} \sim \mathcal{G}_{p \bar{r} \bar{q}}{ }^{\bar{r}} \sim \frac{1}{2} \mathcal{G}_{p \bar{q}} .
$$

Similarly the two scalar curvatures are related to each other: from (2.55), their sum reads exactly,

$$
\begin{aligned}
\mathcal{G}_{p q}{ }^{p q}+\mathcal{G}_{\bar{p} \bar{q}}^{\bar{p} \bar{q}} & =\left(P^{A C} P^{B D}+\bar{P}^{A C} \bar{P}^{B D}\right) S_{A B C D}+\frac{1}{2}\left(P^{C D} \partial_{A} V_{C p} \partial^{A} V_{D}^{p}+\bar{P}^{C D} \partial_{A} \bar{V}_{C \bar{p}} \partial^{A} \bar{V}_{D} \bar{p}\right) \\
& =4 \partial_{A} \partial^{A} d-4 \partial_{A} d \partial^{A} d+\frac{1}{2}\left(\partial_{A} V_{B p} \partial^{A} V^{B p}+\partial_{A} \bar{V}_{B \bar{p}} \partial^{A} \bar{V}^{B \bar{p}}\right)
\end{aligned}
$$

and hence, upon the section condition,

$$
\mathcal{G}_{p q}^{p q}+\mathcal{G}_{\bar{p} \bar{q}}^{\bar{p} \bar{q}} \sim 0
$$

The other formulas in (2.55) also imply a pair of 'trivial' four-index covariant quantities,

$$
\mathcal{G}_{p q \bar{r} \bar{s}}=\mathcal{G}_{A B C D} V_{p}^{A} V^{B}{ }_{q} \bar{V}^{C}{ }_{\bar{r}} \bar{V}^{D}{ }_{\bar{s}} \sim 0, \quad \mathcal{G}_{p \bar{q} r \bar{s}}=\mathcal{G}_{A B C D} V_{p}^{A} \bar{V}^{B}{ }_{\bar{q}} V^{C}{ }_{r} \bar{V}^{D} \bar{s}_{\bar{s}} \sim 0
$$

\subsection{Fermions, Ramond-Ramond cohomology and completely covariant Dirac operators}

- Fermions and Ramond-Ramond cohomology . In addition to the NS-NS sector composed of the dilaton and the pair of vielbeins, the $\mathcal{N}=2 D=10$ supersymmetric extension of DFT [37] calls for a Ramond-Ramond potential, a pair of dilatinos and a pair of gravitinos: the fundamental fields of the supersymmetric theory are precisely,

$$
d, \quad V_{A p}, \quad \bar{V}_{A \bar{p}}, \quad \mathcal{C}_{\bar{\alpha}}^{\alpha}, \quad \rho^{\alpha}, \quad \rho^{\prime \bar{\alpha}}, \quad \psi_{\bar{p}}^{\alpha}, \quad \psi_{p}^{\prime \bar{\alpha}} .
$$

The whole R-R sector is represented by a single potential, $\mathcal{C}^{\alpha}{ }_{\bar{\alpha}}$. As its indices indicate (cf. table 2), it assumes the bi-fundamental spinorial representation of $\operatorname{Spin}(1,9) \times$ $\operatorname{Spin}(9,1)[25,26,38]$.

All the fermions, i.e. dilatinos, gravitinos and supersymmetry parameters, are not twenty, but ten-dimensional Majorana-Weyl spinors. The chirality of the theory reads with two arbitrary sign factors, $\mathbf{c}, \mathbf{c}^{\prime}\left(\mathbf{c}^{2}=\mathbf{c}^{\prime 2}=1\right)$,

$$
\begin{aligned}
& \gamma^{(11)} \psi_{\bar{p}}=\mathbf{c} \psi_{\bar{p}}, \quad \gamma^{(11)} \rho=-\mathbf{c} \rho, \quad \bar{\gamma}^{(11)} \psi_{p}^{\prime}=\mathbf{c}^{\prime} \psi_{p}^{\prime}, \quad \bar{\gamma}^{(11)} \rho^{\prime}=-\mathbf{c}^{\prime} \rho^{\prime}, \\
& \gamma^{(11)} \varepsilon=\mathbf{c} \varepsilon, \quad \bar{\gamma}^{(11)} \varepsilon^{\prime}=\mathbf{c}^{\prime} \varepsilon^{\prime}, \quad \gamma^{(11)} \mathcal{C} \bar{\gamma}^{(11)}=\mathbf{c c}^{\prime} \mathcal{C} .
\end{aligned}
$$


A priori, there are four different sign choices. But, they are all equivalent up to the field redefinitions through $\operatorname{Pin}(1,9) \times \operatorname{Pin}(9,1)$ rotations. That is to say, $\mathcal{N}=2$ $D=10$ SDFT is chiral with respect to both $\mathbf{S p i n}(1,9)$ and $\mathbf{S p i n}(9,1)$, and the theory is unique. Without loss of generality, henceforth we set

$$
\mathbf{c}=+1, \quad \mathbf{c}^{\prime}=+1
$$

Although the theory is unique, the Riemannian solutions are twofold and can be identified as type IIA or IIB supergravity backgrounds [37]. The theory admits also non-Riemannian backgrounds [10] (cf. math literature [49]). The R-R field strength, $\mathcal{F}^{\alpha}{ }_{\bar{\alpha}}$, and its charge conjugation are defined by [38]

$$
\mathcal{F}:=\mathcal{D}_{+} \mathcal{C}, \quad \overline{\mathcal{F}}:=\bar{C}_{+}^{-1}(\mathcal{F})^{T} C_{+} .
$$

Here $\mathcal{D}_{+}$corresponds to one of the two completely covariant differential operators, $\mathcal{D}_{ \pm}$, which are defined by the torsionless connection (2.20) to act on an arbitrary $\operatorname{Spin}(1,9) \times \operatorname{Spin}(9,1)$ bi-fundamental field, $\mathcal{T}^{\alpha}{ }_{\bar{\beta}}$ :

$$
\mathcal{D}_{ \pm} \mathcal{T}:=\gamma^{p} \mathcal{D}_{p} \mathcal{T} \pm \gamma^{(11)} \mathcal{D}_{\bar{p}} \mathcal{T} \bar{\gamma}^{\bar{p}}
$$

where we put $\mathcal{D}_{p}=V^{A}{ }_{p} \mathcal{D}_{A}, \mathcal{D}_{\bar{p}}=\bar{V}^{A}{ }_{\bar{p}} \mathcal{D}_{A}$ and with $(2.38), \mathcal{D}_{A} \mathcal{T}=\partial_{A} \mathcal{T}+\Phi_{A} \mathcal{T}-\mathcal{T} \bar{\Phi}$.

The crucial property of the differential operators, $\mathcal{D}_{ \pm}$, is that upon the section condition they are nilpotent [38]. Straightforward computation can show

$$
\begin{aligned}
\left(\mathcal{D}_{ \pm}\right)^{2} \mathcal{T}= & -\frac{1}{4}\left(\mathcal{G}_{p q}{ }^{p q}+\mathcal{G}_{\bar{p} \bar{q}} \overline{\bar{q}}\right) \mathcal{T}+\partial_{A} \partial^{A} \mathcal{T}-2 \partial_{A} d \partial^{A} \mathcal{T} \\
& +\frac{1}{2} V_{B p} \partial_{A} V^{B}{ }_{q} \gamma^{p q} \partial^{A} \mathcal{T}-\frac{1}{2} \bar{V}_{B \bar{p}} \partial_{A} \bar{V}^{B}{ }_{\bar{q}} \partial^{A} \mathcal{T} \bar{\gamma}^{\bar{p} \bar{q}} \\
& +\frac{1}{4}\left(V_{B p} \partial_{A} \partial^{A} V^{B}{ }_{q}-2 V_{B p} \partial_{A} V^{B}{ }_{q} \partial^{A} d\right) \gamma^{p q} \mathcal{T} \\
& -\frac{1}{4}\left(\bar{V}_{B \bar{p}} \partial_{A} \partial^{A} \bar{V}^{B}{ }_{\bar{q}}-2 V_{B \bar{p}} \partial_{A} \bar{V}^{B}{ }_{\bar{q}} \partial^{A} d\right) \mathcal{T} \bar{\gamma}^{\bar{p} \bar{q}} \\
& +\frac{1}{8} \mathcal{G}_{p q r s} \gamma^{p q r s} \mathcal{T}-\frac{1}{4} \mathcal{G}_{p q \bar{r} \bar{s}} \gamma^{p q} \mathcal{T} \bar{\gamma}^{\bar{r} \bar{s}}+\frac{1}{8} \mathcal{G}_{\bar{p} \bar{q} \bar{r} \bar{s}} \mathcal{T} \bar{\gamma}^{\bar{p} \bar{q} \bar{r} \bar{s}} \\
& \pm \frac{1}{4} \gamma^{(11)}\left(\mathcal{G}_{p \bar{q} \bar{r} \bar{s}} \gamma^{p} \mathcal{T} \bar{\gamma}^{\bar{q} \bar{s} \bar{s}}-\mathcal{G}_{p q r \bar{s}} \gamma^{p q r} \mathcal{T} \bar{\gamma}^{\bar{s}}\right) \\
& \pm \frac{1}{4} \gamma^{(11)}\left[2\left(\mathcal{G}^{\bar{r}}{ }_{p \bar{r} \bar{q}}-\mathcal{G}^{r}{ }_{p r \bar{q}}\right) \gamma^{p} \mathcal{T} \bar{\gamma}^{\bar{q}}-4 V_{B p} \partial_{A} \bar{V}^{B}{ }_{\bar{q}} \gamma^{p} \partial^{A} \mathcal{T} \bar{\gamma}^{\bar{q}}\right]
\end{aligned}
$$

Thus, up to the section condition, with (2.49), (2.56), (2.55), (2.74), each term on the right hand side above vanishes and the nilpotency of the differential operators follows

$$
\left(\mathcal{D}_{ \pm}\right)^{2} \mathcal{T} \sim 0
$$

This defines the R-R cohomology consistently coupled to the NS-NS sector in an $\mathbf{O}(D, D)$ covariant manner [38]. In particular, the $\mathrm{R}-\mathrm{R}$ gauge transformations are given by the same nilpotent differential operator,

$$
\delta \mathcal{C}=\mathcal{D}_{+} \Lambda \longrightarrow \delta \mathcal{F}=\left(\mathcal{D}_{+}\right)^{2} \Lambda \sim 0 .
$$


In a similar fashion to (2.65), upon the section condition, the spin connections transform anomalously under diffeomorphisms,

$$
\left(\delta_{X}-\hat{\mathcal{L}}_{X}\right) \Phi_{A p q} \sim 2 \mathcal{P}_{A p q}{ }^{D E F} \partial_{D} \partial_{[E} X_{F]}, \quad\left(\delta_{X}-\hat{\mathcal{L}}_{X}\right) \bar{\Phi}_{A \bar{p} \bar{q}} \sim 2 \overline{\mathcal{P}}_{A \bar{p} \bar{q}} D E F \partial_{D} \partial_{[E} X_{F]} .
$$

Thus, like (2.67), these anomalous terms can be easily projected out, such that the following modules of the spin connections are completely covariant under diffeomorphisms,

$$
\bar{P}_{A}^{B} \Phi_{B p q}, \quad P_{A}^{B} \bar{\Phi}_{B \bar{p} \bar{q}}, \quad \Phi_{A[p q} V_{r]}^{A}, \quad \bar{\Phi}_{A[\bar{p} \bar{q}} \bar{V}_{\bar{r}]}^{A}, \quad \Phi_{A p q} V^{A p}, \quad \bar{\Phi}_{A \bar{p} \bar{q}} \bar{V}^{A \bar{p}} .
$$

The completely covariant Dirac operators are then, with respect to both diffeomorphisms and local Lorentz symmetries, as follows [36, 42].

$$
\begin{aligned}
& \gamma^{p} \mathcal{D}_{p} \rho=\gamma^{A} \mathcal{D}_{A} \rho, \quad \gamma^{p} \mathcal{D}_{p} \psi_{\bar{p}}, \quad \mathcal{D}_{\bar{p}} \rho, \quad \mathcal{D}_{\bar{p}} \psi^{\bar{p}}=\mathcal{D}_{A} \psi^{A}, \quad \bar{\psi}^{A} \gamma_{p}\left(\mathcal{D}_{A} \psi_{\bar{q}}-\frac{1}{2} \mathcal{D}_{\bar{q}} \psi_{A}\right), \\
& \bar{\gamma}^{\bar{p}} \mathcal{D}_{\bar{p}} \rho^{\prime}=\bar{\gamma}^{A} \mathcal{D}_{A} \rho^{\prime}, \quad \bar{\gamma}^{\bar{p}} \mathcal{D}_{\bar{p}} \psi_{p}^{\prime}, \quad \mathcal{D}_{p} \rho^{\prime}, \quad \mathcal{D}_{p} \psi^{\prime p}=\mathcal{D}_{A} \psi^{\prime A}, \quad \bar{\psi}^{\prime A} \bar{\gamma}_{\bar{p}}\left(\mathcal{D}_{A} \psi_{q}^{\prime}-\frac{1}{2} \mathcal{D}_{q} \psi_{A}^{\prime}\right) .
\end{aligned}
$$

One can also show that $\mathcal{D}_{ \pm} \mathcal{T}(2.81)$ are completely covariant too.

- Completely covariant curvatures from completely covariant derivatives. From (2.30), (2.44) and the relation,

$$
\mathcal{G}_{p \bar{q} A B}=\mathcal{G}_{A B p \bar{q}}=\frac{1}{2}(\mathcal{F}+\overline{\mathcal{F}})_{p \bar{q} A B},
$$

the completely covariant "Ricci" curvatures (2.69), are related to the commutators of the completely covariant differential operators (2.68),

$$
\begin{aligned}
& \mathcal{G}_{p r \bar{q}}{ }^{r} T^{p}=\frac{1}{2} \mathcal{F}_{\bar{q} r p}{ }^{r} T^{p}=\frac{1}{2}\left[\mathcal{D}_{p}, \mathcal{D}_{\bar{q}}\right] T^{p}+\frac{1}{2} \Gamma^{C}{ }_{p \bar{q}} \partial_{C} T^{p} \sim \frac{1}{2}\left[\mathcal{D}_{p}, \mathcal{D}_{\bar{q}}\right] T^{p}, \\
& \mathcal{G}_{p \bar{r} \bar{q}}{ }^{\bar{r}} T^{\bar{q}}=\frac{1}{2} \overline{\mathcal{F}}_{p \bar{r} \bar{q}}{ }^{\bar{r}} T^{\bar{q}}=-\frac{1}{2}\left[\mathcal{D}_{p}, \mathcal{D}_{\bar{r}}\right] T^{\bar{r}}-\frac{1}{2} \Gamma^{C}{ }_{p \bar{q}} \partial_{C} T^{\bar{q}} \sim-\frac{1}{2}\left[\mathcal{D}_{p}, \mathcal{D}_{\bar{q}}\right] T^{\bar{q}} .
\end{aligned}
$$

In a similar fashion to (2.44), we may obtain the expressions for the commutators of the master semi-covariant differential operators which act on spinors, $\varepsilon^{\alpha}$ and $\varepsilon^{\prime \bar{\alpha}}$, in $\operatorname{Spin}(1, D-1)_{L}$ and $\operatorname{Spin}(D-1,1)_{R}$ representations respectively,

$$
\begin{aligned}
{\left[\mathcal{D}_{A}, \mathcal{D}_{B}\right] \varepsilon } & =\frac{1}{4} \mathcal{F}_{A B p q} \gamma^{p q} \varepsilon-\Gamma^{C}{ }_{A B} \partial_{C} \varepsilon, \\
{\left[\mathcal{D}_{A}, \mathcal{D}_{B}\right] \varepsilon^{\prime} } & =\frac{1}{4} \overline{\mathcal{F}}_{A B \bar{p} \bar{q}} \bar{\gamma}^{\bar{p} \bar{q}} \varepsilon^{\prime}-\Gamma^{C}{ }_{A B} \partial_{C} \varepsilon^{\prime} .
\end{aligned}
$$

These immediately imply

$$
\begin{aligned}
& {\left[\gamma^{p} \mathcal{D}_{p}, \mathcal{D}_{\bar{q}}\right] \varepsilon=\mathcal{G}_{p r \bar{q}}{ }^{r} \gamma^{p} \varepsilon-\frac{1}{2} \mathcal{G}_{\bar{q} p r s} \gamma^{p r s} \varepsilon-\Gamma^{C}{ }_{p \bar{q}} \gamma^{p} \partial_{C} \varepsilon,} \\
& {\left[\mathcal{D}_{p}, \bar{\gamma}^{\bar{q}} \mathcal{D}_{\bar{q}}\right] \varepsilon^{\prime}=-\mathcal{G}_{p \bar{r} \bar{q}} \bar{r} \gamma^{\bar{q}} \varepsilon^{\prime}+\frac{1}{2} \mathcal{G}_{p \bar{q} \bar{r} \bar{s}} \bar{\gamma}^{\bar{q} \bar{r} \bar{s}} \varepsilon^{\prime}-\Gamma^{C}{ }_{p \bar{q}} \bar{\gamma}^{\bar{q}} \partial_{C} \varepsilon^{\prime},}
\end{aligned}
$$


and further give

$$
\begin{aligned}
& \gamma^{p q}\left[\mathcal{D}_{p}, \mathcal{D}_{q}\right] \varepsilon=\left(\frac{1}{4} \mathcal{F}_{p q r s} \gamma^{p q r s}-\mathcal{F}_{p r q}{ }^{r} \gamma^{p q}-\frac{1}{2} \mathcal{F}_{p q}{ }^{p q}\right) \varepsilon-\Gamma^{A}{ }_{p q} \gamma^{p q} \partial_{A} \varepsilon \\
& \bar{\gamma}^{\bar{p} \bar{q}}\left[\mathcal{D}_{\bar{p}}, \mathcal{D}_{\bar{p}}\right] \varepsilon^{\prime}=\left(\frac{1}{4} \overline{\mathcal{F}}_{\bar{p} \bar{q} \bar{r} \bar{s}} \bar{\gamma}^{\bar{p} \bar{q} \bar{r} \bar{s}}-\overline{\mathcal{F}}_{\bar{p} \bar{r} \bar{q}} \bar{r} \gamma^{\bar{p} \bar{q}}-\frac{1}{2} \overline{\mathcal{F}}_{\bar{p} \bar{q}} \bar{p} \bar{q}\right) \varepsilon^{\prime}-\Gamma^{A}{ }_{\bar{p} \bar{q}} \bar{\gamma}^{\bar{p} \bar{q}} \partial_{A} \varepsilon^{\prime}
\end{aligned}
$$

Then, combined with the following relations,

$$
\begin{aligned}
\mathcal{D}_{A} \mathcal{D}^{A} \varepsilon= & \partial_{A} \partial^{A} \varepsilon-2 \partial_{A} d \partial^{A} \varepsilon-\frac{1}{8} \Phi_{A p q} \Phi^{A p q} \varepsilon+\frac{1}{4}\left(\mathcal{D}_{A} \Phi^{A}{ }_{p q}\right) \gamma^{p q} \varepsilon \\
& +\frac{1}{2} \Phi_{A p q} \gamma^{p q} \partial^{A} \varepsilon+\frac{1}{16} \Phi_{A p q} \Phi^{A}{ }_{r s} \gamma^{p q r s} \varepsilon \\
\mathcal{D}_{A} \mathcal{D}^{A} \varepsilon^{\prime}= & \partial_{A} \partial^{A} \varepsilon^{\prime}-2 \partial_{A} d \partial^{A} \varepsilon^{\prime}-\frac{1}{8} \bar{\Phi}_{A \bar{p} \bar{q}} \bar{\Phi}^{A \bar{p} \bar{q}} \varepsilon^{\prime}+\frac{1}{4}\left(\mathcal{D}_{A} \bar{\Phi}^{A}{ }_{\bar{p} \bar{q}}\right) \bar{\gamma}^{\bar{p} \bar{q}} \varepsilon^{\prime} \\
& +\frac{1}{2} \bar{\Phi}_{A \bar{p} \bar{q}} \bar{\gamma}^{\bar{p} \bar{q}} \partial^{A} \varepsilon^{\prime}+\frac{1}{16} \bar{\Phi}_{A \bar{p} \bar{q}} \bar{\Phi}^{A}{ }_{\bar{r} \bar{s}} \bar{\gamma}^{\bar{p} \bar{q} \bar{s}} \varepsilon^{\prime}, \\
\mathcal{D}_{A} \Phi^{A}{ }_{p q}= & -2 R_{[p}{ }^{A}{ }_{q] A}+2 \Phi_{A[p}{ }^{r} \Gamma^{A}{ }_{q] r}+\partial_{A}\left(V^{B}{ }_{p} \partial^{A} V_{B q}\right)-2 \partial_{A} d V^{B}{ }_{p} \partial^{A} V_{B q} \\
= & \left.2 \mathcal{F}_{[p}{ }^{r} q\right] r \\
\mathcal{D}_{A} \bar{\Phi}^{A}{ }_{\bar{p} \bar{q}}= & -2 \partial_{A}\left(V_{[\bar{p}}{ }^{A}{ }_{p} \partial^{A} \partial_{A}^{A} V_{B q}+2 \bar{\Phi}_{A[\bar{p}}{ }^{\bar{r}} \Gamma^{A}{ }_{\bar{q}] \bar{r}}+\partial_{A}\left(\partial_{A} d V^{B}{ }_{p} \partial^{A} \partial^{A} V_{B q}, \bar{V}_{B \bar{q}}\right)-2 \partial_{A} d \bar{V}^{B}{ }_{\bar{p}} \partial^{A} \bar{V}_{B \bar{q}}\right. \\
= & 2 \overline{\mathcal{F}}_{[\bar{p} \bar{q} \bar{q}] \bar{r}}+\partial_{A}\left(\bar{V}^{B}{ }_{\bar{p}} \partial^{A} \bar{V}_{B \bar{q}}\right)-2 \partial_{A} d \bar{V}^{B}{ }_{\bar{p}} \partial^{A} \bar{V}_{B \bar{q}},
\end{aligned}
$$

we can derive the following identities,

$$
\begin{aligned}
& {\left[\left(\gamma^{p} \mathcal{D}_{p}\right)^{2}+\mathcal{D}_{\bar{p}} \mathcal{D}^{\bar{p}}\right] \varepsilon=-\frac{1}{4} \mathcal{G}_{p q}{ }^{p q} \varepsilon+\frac{1}{8} \mathcal{G}_{p q r s} \gamma^{p q r s} \varepsilon+\partial_{A} \partial^{A} \varepsilon-2 \partial_{A} d \partial^{A} \varepsilon} \\
& +\frac{1}{4}\left[\partial_{A}\left(V^{B}{ }_{p} \partial^{A} V_{B q}\right)-2 \partial_{A} d V^{B}{ }_{p} \partial^{A} V_{B q}\right] \gamma^{p q} \varepsilon+\frac{1}{2} V^{B}{ }_{p} \partial_{A} V_{B q} \gamma^{p q} \partial^{A} \varepsilon, \\
& {\left[\left(\bar{\gamma}^{\bar{p}} \mathcal{D}_{\bar{p}}\right)^{2}+\mathcal{D}_{p} \mathcal{D}^{p}\right] \varepsilon^{\prime}=-\frac{1}{4} \mathcal{G}_{\bar{p} \bar{q}} \bar{p} \bar{q} \varepsilon^{\prime}+\frac{1}{8} \mathcal{G}_{\bar{p} \bar{q} \bar{r} \bar{s}} \bar{\gamma} \bar{p} \bar{q} \bar{r} \bar{s} \varepsilon^{\prime}+\partial_{A} \partial^{A} \varepsilon^{\prime}-2 \partial_{A} d \partial^{A} \varepsilon^{\prime}} \\
& +\frac{1}{4}\left[\partial_{A}\left(\bar{V}^{B}{ }_{\bar{p}} \partial^{A} \bar{V}_{B \bar{q}}\right)-2 \partial_{A} d \bar{V}^{B}{ }_{\bar{p}} \partial^{A} \bar{V}_{B \bar{q}}\right] \bar{\gamma}^{\bar{p}} \bar{\varepsilon}^{\prime}+\frac{1}{2} \bar{V}_{\bar{p}}^{B} \partial_{A} \bar{V}_{B \bar{q}} \bar{\gamma}^{\bar{p} \bar{q}} \partial^{A} \varepsilon^{\prime} .
\end{aligned}
$$

Therefore, upon the section condition the completely covariant "Ricci" and scalar curvatures (2.69), (2.71) are related to the completely covariant Dirac operators (2.87), cf. Generalized Geometry [25],

$$
\left[\gamma^{p} \mathcal{D}_{p}, \mathcal{D}_{\bar{q}}\right] \varepsilon \sim \mathcal{G}_{p r \bar{q}} \gamma^{p} \varepsilon, \quad\left[\mathcal{D}_{p}, \bar{\gamma}^{\bar{q}} \mathcal{D}_{\bar{q}}\right] \varepsilon^{\prime} \sim-\mathcal{G}_{p \bar{r} \bar{q}} \bar{r} \gamma^{\bar{q}} \varepsilon^{\prime}
$$

and

$$
\left(\gamma^{p} \mathcal{D}_{p}\right)^{2} \varepsilon+\mathcal{D}_{\bar{p}} \mathcal{D}^{\bar{p}} \varepsilon \sim-\frac{1}{4} \mathcal{G}_{p q}{ }^{p q} \varepsilon, \quad\left(\bar{\gamma}^{\bar{p}} \mathcal{D}_{\bar{p}}\right)^{2} \varepsilon^{\prime}+\mathcal{D}_{p} \mathcal{D}^{p} \varepsilon^{\prime} \sim-\frac{1}{4} \mathcal{G}_{\bar{p} \bar{q}} \bar{p} \bar{q} \varepsilon^{\prime} .
$$

While the completely covariant "Ricci" curvatures can be identified from both vectorial and spinorial commutators, (2.89) and (2.95), it appears that the completely covariant scalar curvatures can be only identified in the spinorial representation (2.96). 


\subsection{DFT action and supersymmetric extensions}

- Pure DFT action. The bosonic action of the untwisted DFT for the NS-NS sector, or the pure DFT, is given by the fully covariant scalar curvature,

$$
\int_{\Sigma_{D}} e^{-2 d}\left(P^{A C} P^{B D}-\bar{P}^{A C} \bar{P}^{B D}\right) S_{A B C D}
$$

where the integral is taken over a section, $\Sigma_{D}$. The dilaton and the projector equations of motion correspond to the vanishing of the scalar curvature i.e. the Lagrangian itself and the "Ricci" curvature, $S_{p \bar{q}}$, respectively.

It is precisely this expression of (2.97) that ensures the ' 1.5 formalism' in the full order supersymmetric extensions of DFT with torsionful connections [36, 37]. In fact, without imposing the section condition, the scalar curvature in the Lagrangian (2.97) precisely agrees with the original DFT Lagrangian ([4]) written in terms of the generalized metric, $\mathcal{H}=P-\bar{P}$,

$$
\begin{aligned}
S_{p q}^{p q}-S_{\bar{p} \bar{q}}^{\bar{p} \bar{q}}= & \frac{1}{8} \mathcal{H}^{A B} \partial_{A} \mathcal{H}_{C D} \partial_{B} \mathcal{H}^{C D}+\frac{1}{2} \mathcal{H}^{A B} \partial^{C} \mathcal{H}_{A D} \partial^{D} \mathcal{H}_{B C}-\partial_{A} \partial_{B} \mathcal{H}^{A B} \\
& -4 \mathcal{H}^{A B} \partial_{A} d \partial_{B} d+4 \mathcal{H}^{A B} \partial_{A} \partial_{B} d+4 \partial_{A} \mathcal{H}^{A B} \partial_{B} d .
\end{aligned}
$$

However, due to the relations (2.50), (2.55) which hold for the torsionless connection (2.20), there exist alternative section-condition-equivalent expressions for the action, e.g. $(P-\bar{P})^{A B} S_{A E B}{ }^{E}$ [35], or replacing $S_{A B C D}$ by the spinorial curvature,

$$
\int_{\Sigma_{D}} e^{-2 d}\left(\mathcal{G}_{p q}{ }^{p q}-\mathcal{G}_{\bar{p}} \bar{q} \bar{q} \bar{q}\right) \sim 2 \int_{\Sigma_{D}} e^{-2 d} \mathcal{G}_{p q}{ }^{p q} .
$$

These agree with (2.97) upon the section condition, yet strictly differ by sectioncondition-vanishing purely bosonic terms:

$$
\begin{aligned}
\mathcal{G}_{p q}^{p q}-\mathcal{G}_{\bar{p} \bar{q}}^{\bar{p} \bar{q}} & =S_{p q}^{p q}-S_{\bar{p} \bar{q}}^{\bar{p} \bar{q}}+\frac{1}{2} P^{A B} \partial_{E} V_{A p} \partial^{E} V_{B}^{p}-\frac{1}{2} \bar{P}^{A B} \partial_{E} \bar{V}_{A \bar{p}} \partial^{E} \bar{V}_{B}^{\bar{p}} \\
& =S_{p q}^{p q}-S_{\bar{p} \bar{q}}^{\bar{p} \bar{q}}+\frac{1}{2}\left(\partial_{E} V_{A p} \partial^{E} V^{A p}-\partial_{E} \bar{V}_{A \bar{p}} \partial^{E} \bar{V}^{A \bar{p}}\right)
\end{aligned}
$$

and

$$
2 \mathcal{G}_{p q}^{p q}=S_{p q}^{p q}-S_{\bar{p} \bar{q}}^{\bar{p} \bar{q}}+4 \partial_{A} \partial^{A} d-4 \partial_{A} d \partial^{A} d+\partial_{E} V_{A p} \partial^{E} V^{A p} .
$$

The second equality of (2.100) follows from (2.9), (2.11) and an identity,

$$
\bar{P}^{A B} \partial_{E} V_{A p} \partial^{E} V_{B}^{p}=P^{A B} \partial_{E} \bar{V}_{A \bar{p}} \partial^{E} \bar{V}_{B}^{\bar{p}} .
$$

- The full order supersymmetric extensions. Based on the semi-covariant formalism revisited above, the $\mathcal{N}=2$ (maximal) $D=10$ supersymmetric double field theory has been constructed to the full order in fermions [37],

$$
\mathcal{L}_{D=10}^{\mathcal{N}=2}\left(\mathcal{J}_{A B}, \partial_{A}, d, V_{A p}, \bar{V}_{A \bar{p}}, \mathcal{C}, \rho, \psi_{\bar{p}}, \rho^{\prime}, \psi_{p}^{\prime}\right) .
$$


By truncating the R-R potential and the primed fermions, the $\mathcal{N}=1$ (half-maximal) $D=10$ supersymmetric double field theory $[36]$ is also readily obtainable,

$$
\mathcal{L}_{D=10}^{\mathcal{N}=1}\left(\mathcal{J}_{A B}, \partial_{A}, d, V_{A p}, \bar{V}_{A \bar{p}}, \rho, \psi_{\bar{p}}\right) .
$$

Generically, the supersymmetric double field theory Lagrangians decompose into three parts,

$$
\mathcal{L}_{\mathrm{SDFT}}=\mathcal{L}_{[2,0]}+\mathcal{L}_{[1,2]}+\mathcal{L}_{[0,4]},
$$

where the subscript indices denote the powers of the derivatives and the fermions separately, such that for $\mathcal{L}_{[a, b]}, a+b / 2=2$, as the derivatives and the fermions have the mass dimensions one and half respectively, while the Lagrangian has the mass dimension two. Further, the supersymmetry parameter, $\varepsilon$, has the mass dimension minus half, such that under supersymmetry transformations, each part of the Lagrangian transforms schematically as

$$
\delta_{\varepsilon}\left(\mathcal{L}_{[2,0]}\right)=\Delta_{\varepsilon[2,1]}, \quad \delta_{\varepsilon}\left(\mathcal{L}_{[1,2]}\right)=\Delta_{\varepsilon[2,1]}^{\prime}+\Delta_{\varepsilon[1,3]}, \quad \delta_{\varepsilon}\left(\mathcal{L}_{[0,4]}\right)=\Delta_{\varepsilon[1,3]}^{\prime}+\Delta_{\varepsilon[0,5]},
$$

where $a+b / 2=5 / 2$ for each $\Delta_{\varepsilon[a, b]}^{(\prime)}$.

In particular, the supersymmetry of the $\mathcal{N}=1, D=10$ SDFT [36] amounts to the following algebraic identities,

$$
\begin{aligned}
\Delta_{\varepsilon[2,1]}+\Delta_{\varepsilon[2,1]}^{\prime} & =\partial_{A} K_{[1,1]}^{A}+\left[\bullet \partial_{A} \partial^{A} \bullet+\partial_{A} \bullet \partial^{A} \bullet\right], \\
\Delta_{\varepsilon[1,3]}+\Delta_{\varepsilon[1,3]}^{\prime} & =\partial_{A} K_{[0,3]}^{A}, \\
\Delta_{\varepsilon[0,5]} & =0,
\end{aligned}
$$

such that the Lagrangian is invariant up to total derivatives and the section condition,

$$
\delta_{\varepsilon} \mathcal{L}_{D=10}^{\mathcal{N}=1}=\partial_{A}\left(K_{[1,1]}^{A}+K_{[0,3]}^{A}\right)+\left[\bullet \partial_{A} \partial^{A} \bullet+\partial_{A} \bullet \partial^{A} \bullet\right]_{[2,1]} .
$$

On the other hand, the supersymmetry of the $\mathcal{N}=2 D=10$ SDFT [37] means the invariance of the Lagrangian up to total derivatives, the section condition and the self-duality of the R-R field strength,

$$
\delta_{\varepsilon} \mathcal{L}_{D=10}^{\mathcal{N}=2}=-\frac{1}{8} e^{-2 d} \bar{V}^{A}{ }_{\bar{q}} \delta_{\varepsilon} V_{A p} \operatorname{Tr}\left(\gamma^{p} \tilde{\mathcal{F}}_{-} \bar{\gamma}^{\bar{q}} \tilde{\mathcal{F}}_{-}\right)+\partial_{A} K^{A}+\left[\bullet \partial_{A} \partial^{A} \bullet+\partial_{A} \bullet \partial^{A} \bullet\right]_{[2,1]},
$$

where $\tilde{\mathcal{F}}_{-}$is the self-dual part of the R-R field strength (2.80) defined, to the full order in fermions, by

$$
\tilde{\mathcal{F}}_{-}:=\left(1-\gamma^{(11)}\right)\left(\mathcal{F}-i \frac{1}{2} \rho \bar{\rho}^{\prime}+i \frac{1}{2} \gamma^{p} \psi_{\bar{q}} \bar{\psi}_{p}^{\prime} \bar{\gamma}^{\bar{q}}\right),
$$

and $\overline{\tilde{\mathcal{F}}_{-}}$denotes, like $(2.80)$, its charge conjugation,

$$
\overline{\tilde{\mathcal{F}}_{-}}=\bar{C}_{+}^{-1}\left(\tilde{\mathcal{F}}_{-}\right)^{T} C_{+} .
$$


A crucial fact about the section-condition-vanishing terms, $\left[\bullet \partial_{A} \partial^{A} \bullet+\partial_{A} \bullet \partial^{A} \bullet\right]_{[2,1]}$, which is common in (2.108) and (2.109), is that they are strictly linear in the fermions (dilatinos and gravitinos), and hence they are fully obtainable just from the leading order supersymmetry transformation rules.

It is also crucial to note that the above form of the algebraic identities, (2.108) and (2.109), still holds, i.e. the supersymmetry is unbroken upon the section condition, even if we deform the Lagrangian by adding arbitrary section-conditionvanishing terms, which, counting the mass dimensions, should be purely bosonic. Examples include the replacement of $S_{A B C D}$ by $\mathcal{G}_{A B C D}$ and adding $\mathcal{G}_{p q}{ }^{p q}+\mathcal{G}_{\bar{p} \bar{q}} \bar{p} \bar{q}(2.74)$ to the $\mathcal{N}=1$ (but not $\mathcal{N}=2$ ) $D=10$ SDFT Lagrangian, which we shall take below, for the supersymmetry preserving twist.

\section{U-twisted double field theory}

Here we twist the double field theory formulated within the semi-covariant formalism. Our twist is a DFT generalization of the Scherk-Schwarz twist, based on [21, 22, 27, 28], and will be from time to time referred to as $U$-twist. In section 3.1, we introduce our ansatz of the twist. It involves a scalar and a local $\mathbf{O}(D, D)$ group element which may not obey the section condition. In section 3.3, following closely Grana and Marques [27], from the closure of the U-twisted generalized Lie derivative we derive a set of consistency conditions which we call twistability conditions. They generalize the original section condition and slightly differ from [27]. In section 3.4, we perform the U-twist on the semi-covariant formalism and verify that, with the replacement of $S_{A B C D}$ by $\mathcal{G}_{A B C D}$, essentially all the nice properties of the semi-covariant formalism, including the complete covariantizability, survive after the twist, subject to the twistability conditions. We also verify that both the $\mathcal{N}=2$ supersymmetric invariance and the nilpotency of the differential operators which define the twisted Ramond-Ramond cohomology commonly require an extra condition. Consequently, the maximal supersymmetric twist of the $\mathcal{N}=2 D=10$ SDFT requires one more twistability condition compared to the half-maximal supersymmetric twist of the $\mathcal{N}=1 D=10$ SDFT.

\subsection{Ansatz for U-twist}

U-twist calls for two local variables, or the twisting data: a scalar function, $\lambda(x)$, and an $\mathbf{O}(D, D)$ element, $U(x)$. Both of them do not necessarily satisfy the section condition (2.3), but shall be required to meet consistency conditions, or the twistability conditions.

The local $\mathbf{O}(D, D)$ element, $U_{M}{ }^{\dot{N}}$, carries one undotted (untwisted) row index and the other dotted (twisted) column index, such that, with the introduction of an additional $\mathbf{O}(D, D)$ invariant metric,

$$
\dot{\mathcal{J}}_{\dot{M} \dot{N}}=\left(\begin{array}{ll}
0 & 1 \\
1 & 0
\end{array}\right),
$$

it satisfies

$$
U \dot{\mathcal{J}} U^{t}=\mathcal{J}
$$


While the dotted (twisted) metric, $\dot{\mathcal{J}}_{\dot{M} \dot{N}}$, may coincide numerically with the undotted (untwisted) metric, $\mathcal{J}_{M N}$ (cf. table 2), hereafter we deliberately distinguish them. In particular, the two different kinds of indices will never be contracted.

$U$-twist prescribes substituting the following expression for each untwisted (undotted) field, $T_{A_{1} \cdots A_{n}}$, into the $D=10$ ungauged DFT Lagrangians, cf. [27],

$$
T_{A_{1} \cdots A_{n}}=e^{-2 \omega \lambda} U_{A_{1}} \dot{A}_{1} \cdots U_{A_{n}}{ }^{\dot{A}_{n}} \dot{T}_{\dot{A}_{1} \cdots \dot{A}_{n}} .
$$

Equivalently, twisted fields are defined to carry dotted $\mathbf{O}(D, D)$ indices with a relevant weight factor,

$$
\dot{T}_{\dot{A}_{1} \cdots \dot{A}_{n}}:=e^{2 \omega \lambda}\left(U^{-1}\right)_{\dot{A}_{1}}{ }^{B_{1}} \cdots\left(U^{-1}\right)_{\dot{A}_{n}}{ }^{B_{n}} T_{B_{1} \cdots B_{n}} .
$$

The derivatives of the untwisted fields then assume a generic form,

$$
\partial_{C} T_{A_{1} \cdots A_{n}}=e^{-2 \omega \lambda} U_{C} \dot{C}_{A_{A_{1}}}^{\dot{A}_{1}} \cdots U_{A_{n}}^{\dot{A}_{n}} \dot{D}_{\dot{C}} \dot{T}_{\dot{A}_{1} \cdots \dot{A}_{n}},
$$

which naturally leads to the definition of what we call $U$-derivative: with the pull-back of the naked derivative,

$$
\dot{\partial}_{\dot{C}}=U^{-1}{ }_{\dot{C}}^{C} \partial_{C},
$$

and a pure gauge "connection",

$$
\Omega_{\dot{C} \dot{A}} \dot{B}:=\left(U^{-1} \dot{\partial}_{\dot{C}} U\right)_{\dot{A}} \dot{B},
$$

the U-derivative, $\dot{D}_{\dot{C}}$, is defined to act on a twisted field by

$$
\dot{D}_{\dot{C}} \dot{T}_{\dot{A}_{1} \cdots \dot{A}_{n}}:=\dot{\partial}_{\dot{C}} \dot{T}_{\dot{A}_{1} \cdots \dot{A}_{n}}-2 \omega \dot{\partial}_{\dot{C}} \lambda \dot{T}_{\dot{A}_{1} \cdots \dot{A}_{n}}+\sum_{i=1}^{n} \Omega_{\dot{C}_{A_{i}}}{ }^{\dot{B}} \dot{T}_{\dot{A}_{1} \cdots \dot{B} \cdots \dot{A}_{n}}
$$

In particular, the twist of the $\mathcal{N}=1$ or the $\mathcal{N}=2, D=10$ SDFT amounts to inserting the following expressions for the dilaton and the vielbeins into the untwisted Lagrangian,

$$
e^{-2 d}=e^{-2 \lambda} e^{-2 \dot{d}}, \quad V_{A p}=U_{A} \dot{A}_{\dot{A}} \dot{V}_{\dot{A} p}, \quad \bar{V}_{A \bar{p}}=U_{A} \dot{A}_{\bar{V}}^{\dot{V}_{\dot{A} \bar{p}}} .
$$

They are the only field variables to be twisted, since other fields (fermions and the $\mathrm{R}-\mathrm{R}$ potential) are weightless and $\mathbf{O}(D, D)$ singlet. We shall not put a dot on those effectively untwisted fields for simplicity. The replacement naturally leads to the twisted, half-maximal or maximal SDFT Lagrangians, cf. (4.1), (4.7),

$$
\begin{gathered}
\mathcal{L}_{D=10}^{\mathcal{N}=1}\left(\mathcal{J}_{A B}, \partial_{A}, d, V_{A p}, \bar{V}_{A \bar{p}}, \rho, \psi_{\bar{p}}\right)=e^{-2 \lambda} \dot{\mathcal{L}}_{\text {Twisted SDFT }}^{\text {Half-maximal }}\left(\dot{\mathcal{J}}_{\dot{A} \dot{B}}, \dot{D}_{\dot{A}}, \dot{d}, \dot{V}_{\dot{A} p}, \dot{\bar{V}}_{\dot{A} \bar{p}}, \rho, \psi_{\bar{p}}\right), \\
\mathcal{L}_{D=10}^{\mathcal{N}=2}\left(\mathcal{J}_{A B}, \partial_{A}, d, V_{A p}, \bar{V}_{A \bar{p}}, \mathcal{C}, \rho, \psi_{\bar{p}}, \rho^{\prime}, \psi_{p}^{\prime}\right)=e^{-2 \lambda} \dot{\mathcal{L}}_{\mathrm{Tw} \text { wisted SDFT }}^{\text {Maximal }}\left(\dot{\mathcal{J}}_{\dot{A} \dot{B}}, \dot{D}_{\dot{A}}, \dot{d}, \dot{V}_{\dot{A}_{p}}, \dot{\bar{V}}_{\dot{A} \bar{p}}, \mathcal{C}, \rho, \psi_{\bar{p}}, \rho^{\prime}, \psi_{p}^{\prime}\right) .
\end{gathered}
$$

As seen from the right hand sides of the equalities, - since every DFT Lagrangian is $\mathbf{O}(D, D)$ singlet and possesses the diffeomorphic weight of unity - after the replacement, $i)$ the twisting matrix, $U_{A} \dot{A}$, effectively drops out in the Lagrangian, ii) the $\mathbf{O}(D, D)$ invariant constant metric gets 'dotted', and iii) the naked derivatives become the U-derivatives. 
The twist translates the original section condition as

$$
\dot{D}_{\dot{A}} \dot{D}^{\dot{A}} \sim 0
$$

However, we do not intend to impose this condition on the twisted theory. Imposing this would lead to a mere equivalent reformulation of the untwisted double field theory where (3.3) corresponded to field redefinition. In section 3.3, we shall look for alternative inequivalent conditions, or the twistability conditions. Before doing so, in the next subsection we pause to collect some useful properties of the U-derivative, $\dot{D}_{\dot{C}}$.

\subsection{Properties of the U-derivative and its connection}

Since $U_{A} \dot{B}$ is an $\mathbf{O}(D, D)$ element, we have

$$
\Omega_{\dot{C} \dot{A} \dot{B}}+\Omega_{\dot{C} \dot{B} \dot{A}}=\dot{\partial}_{\dot{C}}\left(\dot{\mathcal{J}} U^{t} \mathcal{J}^{-1} U \dot{\mathcal{J}}\right)_{\dot{A} \dot{B}}=\dot{\partial}_{\dot{C}} \dot{\mathcal{J}}_{\dot{A} \dot{B}}=0
$$

Hence, the U-derivative "connection" is skew-symmetric for the last two indices,

$$
\Omega_{\dot{C} \dot{A} \dot{B}}=-\Omega_{\dot{C} \dot{B} \dot{A}}=\Omega_{\dot{C}[\dot{A} \dot{B}]} .
$$

It is worth while to note

$$
\Omega_{\dot{B}}^{\dot{B} \dot{A}}=\partial^{B} U_{B}^{\dot{A}}, \quad \Omega^{\dot{B}}{ }_{\dot{B} \dot{A}}=\partial_{B}\left(U^{-1}\right)_{\dot{A}}{ }^{B},
$$

and $^{6}$

$$
\dot{\partial}_{\dot{A}} \Omega_{\dot{B}} \dot{B} \dot{A}=-\frac{1}{2} \Omega_{\dot{A}} \dot{A} \dot{B} \Omega^{\dot{C}} \dot{C} \dot{B}-\frac{1}{2} \Omega_{\dot{C} \dot{B} \dot{A}} \Omega^{\dot{B} \dot{C} \dot{A}} .
$$

Pulling back the dotted derivative index to a undotted index, it is useful to consider

$$
D_{C} \dot{T}_{\dot{A}_{1} \cdots \dot{A}_{n}}=U_{C} \dot{C} \dot{D}_{\dot{C}} \dot{T}_{\dot{A}_{1} \cdots \dot{A}_{n}}=\partial_{C} \dot{T}_{\dot{A}_{1} \cdots \dot{A}_{n}}-2 \omega \partial_{C} \lambda \dot{T}_{\dot{A}_{1} \cdots \dot{A}_{n}}+\sum_{i=1}^{n} \Omega_{C \dot{A}_{i}} \dot{B}_{\dot{T}_{\dot{A}_{1} \cdots \dot{B} \cdots \dot{A}_{n}}}
$$

where naturally we put

$$
\Omega_{C \dot{A}} \dot{B}=U_{C}{ }^{\dot{C}} \Omega_{\dot{C} \dot{A}} \dot{B}=\left(U^{-1} \partial_{C} U\right)_{\dot{A}}^{\dot{B}} .
$$

This corresponds to "pure gauge" and thus its "field strength" vanishes identically,

$$
\begin{aligned}
0 & =\partial_{A} \Omega_{B \dot{C}} \dot{D}-\partial_{B} \Omega_{A \dot{C}} \dot{D}+\Omega_{A \dot{C}} \dot{E} \Omega_{B \dot{E}} \dot{D}-\Omega_{B \dot{C}} \dot{E} \Omega_{A \dot{E}} \dot{D} \\
& =D_{A} \Omega_{B \dot{C}} \dot{D}-D_{B} \Omega_{A \dot{C}} \dot{D}-\Omega_{A \dot{C}} \Omega_{B \dot{E}}^{\dot{D}}+\Omega_{B \dot{C}} \Omega_{A \dot{E}}^{\dot{D}} .
\end{aligned}
$$

By construction, the U-derivative (3.8) can be rewritten as

$$
\dot{D}_{\dot{C}} \dot{T}_{\dot{A}_{1} \cdots \dot{A}_{n}}=e^{2 \omega \lambda}\left(U^{-1}\right)_{\dot{C}}{ }^{C}\left(U^{-1}\right)_{\dot{A}_{1}}{ }^{A_{1}} \cdots\left(U^{-1}\right)_{\dot{A}_{n}}{ }^{A_{n}} \partial_{C}\left(e^{-2 \omega \lambda} U_{A_{1}}{ }^{\dot{B}_{1}} \cdots U_{A_{n}}{ }^{\dot{B}_{n}} \dot{T}_{\dot{B}_{1} \cdots \dot{B}_{n}}\right),
$$

\footnotetext{
${ }^{6}$ Eq. (3.15) can be derived from the following manipulation,

$\dot{\partial}_{\dot{A}} \Omega_{\dot{B}}{ }^{\dot{B} \dot{A}}=U^{B}{ }_{\dot{A}} \partial_{B} \partial_{C} U^{C \dot{A}}=\partial_{C}\left(U_{\dot{B}}^{C} \Omega_{\dot{A} \dot{B}}{ }^{\dot{A}}\right)-\Omega_{\dot{C} \dot{B} \dot{A}} \Omega^{\dot{B} \dot{C} \dot{A}}=-\dot{\partial}_{\dot{B}} \Omega_{\dot{A}}{ }^{\dot{A} \dot{B}}-\Omega_{\dot{A}}{ }^{\dot{A} \dot{B}} \Omega^{\dot{C}}{ }_{\dot{C} \dot{B}}-\Omega_{\dot{C} \dot{B} \dot{A}} \Omega^{\dot{B} \dot{C} \dot{A}}$.
} 
and is compatible with the $U$ matrix itself,

$$
\begin{array}{rlrl}
D_{A} U_{B} \dot{C} & =\partial_{A} U_{B} \dot{C}-U_{B}{ }^{\dot{D}} \Omega_{A \dot{D}} \dot{C}=0, & \dot{D}_{\dot{A}} U_{B} \dot{C}=0, \\
D_{A}\left(U^{-1}\right)_{\dot{C}}{ }^{B}=\partial_{A}\left(U^{-1}\right)_{\dot{C}}{ }^{B}+\Omega_{A \dot{C}}{ }^{\dot{D}}\left(U^{-1}\right)_{\dot{D}}{ }^{B}=0, & \dot{D}_{\dot{A}}\left(U^{-1}\right)_{\dot{C}}{ }^{B}=0,
\end{array}
$$

as well as with both the dotted and the undotted $\mathbf{O}(D, D)$ invariant metrics,

$$
D_{C} \dot{\mathcal{J}}_{\dot{A} \dot{B}}=2 \Omega_{C(\dot{A} \dot{B})}=0, \quad \dot{D}_{\dot{C}} \dot{\mathcal{J}}_{\dot{A} \dot{B}}=0, \quad \dot{D}_{\dot{C}} \mathcal{J}_{A B}=\dot{\partial}_{\dot{C}} \mathcal{J}_{A B}=0
$$

Pushing back the undotted indices of (3.18) to the dotted ones, utilizing the above $U$ matrix compatibility (3.20), we also get another useful relation,

$$
\dot{D}_{[\dot{A}} \Omega_{\dot{B}]} \dot{C} \quad \dot{D}=\Omega_{[\dot{A}} \dot{C} \dot{E} \Omega_{\dot{B}] \dot{E} \dot{D}} .
$$

We emphasize that, the $U$ matrix compatibility is only possible because we distinguish the dotted and the undotted $\mathbf{O}(D, D)$ indices deliberately.

Furthermore, from

$$
D_{A} D_{B} \dot{T}_{\dot{C}_{1} \cdots \dot{C}_{n}}=e^{2 \omega \lambda}\left(U^{-1}\right)_{\dot{C}_{1}}{ }^{C_{1}} \cdots\left(U^{-1}\right)_{\dot{C}_{n}}{ }^{C_{n}} \partial_{A} \partial_{B}\left(e^{-2 \omega \lambda} U_{C_{1}} \dot{E}_{1} \cdots U_{C_{n}} \dot{E}_{n} \dot{T}_{\dot{E}_{1} \cdots \dot{E}_{n}}\right) \text {, }
$$

the U-derivatives are all commutative,

$$
\left[D_{A}, D_{B}\right]=0, \quad\left[D_{A}, \dot{D}_{\dot{B}}\right]=0, \quad\left[\dot{D}_{\dot{A}}, \dot{D}_{\dot{B}}\right]=0 .
$$

This is a crucial result. It means that there is no ordering ambiguity of the U-derivatives, as one might worry while performing the twist, (3.10). Namely, the 'field strength' and the 'torsion' of the U-derivative are all trivial. It is also worth while to compare with the Weitzenböck connection, e.g. [30]. Although it appears formally similar to our $\Omega$, there is a crucial difference: we intentionally distinguish the dotted indices from the undotted indices, while the Weitzenböck connection and hence the corresponding Weitzenböck derivative do not. Consequently, the Weitzenböck derivatives do not commute, unlike (3.24), and the Weizenböck connection is torsionful.

The dilaton, $d$, corresponds to the logarithm of a weightful scalar density. Its Uderivative is then determined from

$$
\partial_{A} e^{-2 d}=e^{-2 \lambda} D_{A} e^{-2 \dot{d}}=-2\left(D_{A} \dot{d}\right) e^{-2 \lambda-2 \dot{d}},
$$

by

$$
D_{A} \dot{d}=\partial_{A} \dot{d}+\partial_{A} \lambda=\partial_{A} d, \quad \dot{D}_{\dot{A}} \dot{d}=\dot{\partial}_{\dot{A}} \dot{d}+\dot{\partial}_{\dot{A}} \lambda=\dot{\partial}_{\dot{A}} d .
$$

Further, its second order derivatives are ${ }^{7}$

$$
\partial_{A} \partial_{B} d=\partial_{A}\left(U_{B}^{\dot{B}} \dot{D}_{\dot{B}} \dot{d}\right)=D_{A}\left(U_{B} \dot{B} \dot{D}_{\dot{B}} \dot{d}\right)=U_{A}{ }^{\dot{A}} U_{B} \dot{B}_{\dot{D}} \dot{D}_{\dot{B}} \dot{d}=D_{A} D_{B} \dot{d}
$$

\footnotetext{
${ }^{7}$ This is also consistent with the following manipulation,

$\left(-2 \partial_{A} \partial_{B} d+4 \partial_{A} d \partial_{B} d\right) e^{-2 d}=\partial_{A} \partial_{B} e^{-2 d}=\partial_{A}\left(e^{-2 \lambda} D_{B} e^{-2 \dot{d}}\right)=e^{-2 \lambda}\left(\partial_{A}-2 \partial_{A} \lambda\right) D_{B} e^{-2 \dot{d}}$ $=e^{-2 \lambda} D_{A} D_{B} e^{-2 \dot{d}}=e^{-2 \lambda}\left(-2 D_{A} D_{B} \dot{d}+4 D_{A} \dot{d} D_{B} \dot{d}\right) e^{-2 \dot{d}}$.
} 
and thus, in general,

$$
\partial_{A_{1}} \partial_{A_{2}} \cdots \partial_{A_{n}} d=U_{A_{1}}{ }^{\dot{A}_{1}} U_{A_{2}} \dot{A}_{2} \cdots U_{A_{n}} \dot{A}_{n} \dot{D}_{\dot{A}_{1}} \dot{D}_{\dot{A}_{2}} \cdots \dot{D}_{\dot{A}_{n}} \dot{d}=D_{A_{1}} D_{A_{2}} \cdots D_{A_{n}} \dot{d}
$$

Now, following [27], we define two key quantities out of the twisting data,

$$
f_{\dot{A}}:=\Omega^{\dot{B}}{ }_{\dot{B} \dot{A}}-2 \dot{\partial}_{\dot{A}} \lambda=\partial_{C} U_{\dot{A}}^{C}-2 \dot{\partial}_{\dot{A}} \lambda
$$

and the 'structure constant',

$$
f_{\dot{A} \dot{B} \dot{C}}:=\Omega_{\dot{A} \dot{B} \dot{C}}+\Omega_{\dot{B} \dot{C} \dot{A}}+\Omega_{\dot{C} \dot{A} \dot{B}}=f_{[\dot{A} \dot{B} \dot{C}]}
$$

Through straightforward computations, one can verify

$$
\dot{\partial}^{\dot{C}} \Omega_{\dot{C} \dot{A} \dot{B}}=\dot{\partial} \dot{C} f_{\dot{C} \dot{A} \dot{B}}+\dot{\partial}_{\dot{A}} f_{\dot{B}}-\dot{\partial}_{\dot{B}} f_{\dot{A}}+f^{\dot{C}} \Omega_{\dot{A} \dot{B} \dot{C}}-f^{\dot{C}} \Omega_{\dot{B} \dot{A} \dot{C}},
$$

and

$$
\begin{aligned}
f_{\dot{A} \dot{B} \dot{E}} f_{\dot{C} \dot{D}} \dot{E}= & \Phi_{\dot{A} \dot{B} \dot{C} \dot{D}}-\Phi_{\dot{B} \dot{A} \dot{C} \dot{D}}+\Phi_{\dot{A} \dot{C} \dot{B} \dot{D}}-\Phi_{\dot{B} \dot{C} \dot{A} \dot{D}}+\Phi_{\dot{B} \dot{A} \dot{D} \dot{C}}-\Phi_{\dot{A} \dot{B} \dot{D} \dot{C}}+\Phi_{\dot{C} \dot{A} \dot{D} \dot{B}}-\Phi_{\dot{C} \dot{B} \dot{D} \dot{A}} \\
& +\Omega_{E \dot{A} \dot{B}} \Omega^{E}{ }_{\dot{C} \dot{D}}-\dot{\partial}_{\dot{C}} \Omega_{\dot{D} \dot{A} \dot{B}}+\dot{\partial}_{\dot{D}} \Omega_{\dot{C} \dot{A} \dot{B}}-\dot{\partial}_{\dot{A}} \Omega_{\dot{B} \dot{C} \dot{D}}+\dot{\partial}_{\dot{B}} \Omega_{\dot{A} \dot{C} \dot{D}},
\end{aligned}
$$

where we set

$$
\Phi_{\dot{A} \dot{B} \dot{C} \dot{D}}=\dot{\partial}_{\dot{A}} U^{E}{ }_{\dot{B}} \dot{\partial}_{\dot{C}} U_{E \dot{D}}=\Phi_{\dot{C} \dot{D} \dot{A} \dot{B}}
$$

In particular, this implies

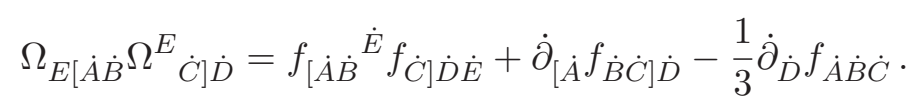

We shall make use of these identities shortly below.

Finally, from (3.5), the divergence of a vector density with weight one becomes after the twist,

$$
\partial_{A} K^{A}=e^{-2 \lambda} \dot{D}_{\dot{A}} \dot{K}^{\dot{A}}=e^{-2 \lambda}\left(\dot{\partial}_{\dot{A}} \dot{K}^{\dot{A}}+f_{\dot{A}} \dot{K}^{\dot{A}}\right) .
$$

Thus, after the twist, the potentially anomalous terms in the supersymmetric variations of the $\mathcal{N}=1$ or the $\mathcal{N}=2 D=10$ SDFT Lagrangian (2.108), (2.109) assume the following generic form,

$$
\left\langle\delta_{\varepsilon} \dot{\mathcal{L}}_{\mathrm{SDFT}}^{\text {Twisted }}\right\rangle_{\text {anomalous }}=f_{\dot{A}} \dot{K}^{\dot{A}}+\left[\bullet \dot{D}_{\dot{A}} \dot{D}^{\dot{A}} \dot{\bullet}+\dot{D}_{A} \dot{\bullet} \dot{D}^{\dot{A}} \bullet\right]_{[2,1]} .
$$

In order to ensure the supersymmetry to be unbroken after the twist, we need to show that these terms vanish up to the twistability conditions. Fortunately, as discussed in section 2.5 and demonstrated in section 4 later, these anomalous terms can be all sufficiently obtained just from the leading order supersymmetry. 


\subsection{Twistability conditions: closure of the diffeomorphisms}

Acting on the dotted twisted fields, twisted diffeomorphism is generated by the U-twisted generalized Lie derivative,

$\dot{\mathcal{L}}_{\dot{X}} \dot{T}_{\dot{A}_{1} \cdots \dot{A}_{n}}:=\dot{X}^{\dot{B}} \dot{D}_{\dot{B}} \dot{T}_{\dot{A}_{1} \cdots \dot{A}_{n}}+\omega \dot{D}_{\dot{B}} \dot{X}^{\dot{B}} \dot{T}_{\dot{A}_{1} \cdots \dot{A}_{n}}+\sum_{i=1}^{n}\left(\dot{D}_{\dot{A}_{i}} \dot{X}_{\dot{B}}-\dot{D}_{\dot{B}} \dot{X}_{\dot{A}_{i}}\right) \dot{T}_{\dot{A}_{1} \cdots \dot{A}_{i-1}}{ }_{\dot{A}_{i+1} \cdots \dot{A}_{n}}$

In an identical manner to the twisting ansatz (3.4), this expression is related to the untwisted generalized Lie derivative (2.4) by

$$
\dot{\mathcal{L}}_{\dot{X}} \dot{T}_{\dot{A}_{1} \cdots \dot{A}_{n}}=e^{2 \omega \lambda}\left(U^{-1}\right)_{\dot{A}_{1}}{ }^{A_{1}} \cdots\left(U^{-1}\right)_{\dot{A}_{n}}{ }^{A_{n}} \hat{\mathcal{L}}_{X} T_{A_{1} \cdots A_{n}}
$$

The commutator of the U-twisted generalized Lie derivatives, without employing any section condition, reads readily from (2.7), cf. Grana and Marques [27],

$$
\begin{aligned}
\left(\left[\dot{\mathcal{L}}_{\dot{X}}, \dot{\mathcal{L}}_{\dot{Y}}\right]-\dot{\mathcal{L}}_{[\dot{X}, \dot{Y}]_{\dot{\mathrm{C}}}}\right) \dot{T}_{\dot{A}_{1} \cdots \dot{A}_{n}}= & \frac{1}{2}\left(\dot{X}^{\dot{N}} \dot{D}^{\dot{M}} \dot{Y}_{\dot{N}}-\dot{Y}^{\dot{N}} \dot{D}^{\dot{M}} \dot{X}_{\dot{N}}\right) \dot{D}_{\dot{M}} \dot{T}_{\dot{A}_{1} \cdots \dot{A}_{n}} \\
& +\frac{1}{2} \omega\left(\dot{X}^{\dot{N}} \dot{D}_{\dot{M}} \dot{D}^{\dot{M}} \dot{Y}_{\dot{N}}-\dot{Y}^{\dot{N}} \dot{D}_{\dot{M}} \dot{D}^{\dot{M}} \dot{X}_{\dot{N}}\right) \dot{T}_{\dot{A}_{1} \cdots \dot{A}_{n}} \\
& +\sum_{i=1}^{n}\left(\dot{D}_{\dot{M}} \dot{Y}_{\dot{A}_{i}} \dot{D}^{\dot{M}} \dot{X}_{\dot{B}}-\dot{D}_{\dot{M}} \dot{X}_{\dot{A}_{i}} \dot{D}^{M} \dot{Y}_{\dot{B}}\right) \dot{T}_{\dot{A}_{1} \cdots \dot{A}_{i-1}}{ }_{\dot{B}} \dot{A}_{i+1} \cdots \dot{A}_{n}
\end{aligned}
$$

where $[\dot{X}, \dot{Y}]_{\dot{\mathrm{C}}}$ denotes the U-twisted C-bracket,

$$
[\dot{X}, \dot{Y}]_{\dot{\mathrm{C}}}^{\dot{A}}:=\dot{X}^{\dot{B}} \dot{D}_{\dot{B}} \dot{Y}^{\dot{A}}-\dot{Y}^{\dot{B}} \dot{D}_{\dot{B}} \dot{X}^{\dot{A}}+\frac{1}{2} \dot{Y}^{\dot{B}} \dot{D}^{\dot{A}} \dot{X}_{\dot{B}}-\frac{1}{2} \dot{X}^{\dot{B}} \dot{D}^{\dot{A}} \dot{Y}_{\dot{B}}
$$

Clearly, if the condition of (3.11) were imposed, the right hand side of (3.39) would vanish. Yet, we are after other way of ensuring the closure. To this end, we dismantle the U-derivative and display its connection explicitly: the U-twisted generalized Lie derivative (3.37) and the U-twisted C-bracket (3.39) can be rewritten, in terms of $f_{\dot{A}}(3.29)$ and $f_{\dot{A} \dot{B} \dot{C}}(3.30)$, as

$$
\begin{aligned}
\dot{\mathcal{L}}_{\dot{X}} \dot{T}_{\dot{A}_{1} \cdots \dot{A}_{n}}= & \dot{X}^{\dot{B}} \dot{\partial}_{\dot{B}} \dot{T}_{\dot{A}_{1} \cdots \dot{A}_{n}}+\omega\left(\dot{\partial}_{\dot{B}} \dot{X}^{\dot{B}}+f_{\dot{B}} \dot{X}^{\dot{B}}\right) \dot{T}_{\dot{A}_{1} \cdots \dot{A}_{n}} \\
& +\sum_{i=1}^{n}\left(\dot{\partial}_{\dot{A}_{i}} \dot{X}_{\dot{B}}-\dot{\partial}_{\dot{B}} \dot{X}_{\dot{A}_{i}}+f_{\dot{A}_{i} \dot{B} \dot{C}} \dot{X}^{\dot{C}}\right) \dot{T}_{\dot{A}_{1} \cdots \dot{A}_{i-1}} \dot{B}_{A_{i+1} \cdots \dot{A}_{n}},
\end{aligned}
$$

and

$$
[\dot{X}, \dot{Y}]_{\mathrm{C}}^{\dot{A}}=\dot{X}^{\dot{B}} \dot{\partial}_{\dot{B}} \dot{Y}^{\dot{A}}-\dot{Y}^{\dot{B}} \dot{\partial}_{\dot{B}} \dot{X}^{\dot{A}}+\frac{1}{2} \dot{Y}^{\dot{B}} \dot{\partial}^{\dot{A}} \dot{X}_{\dot{B}}-\frac{1}{2} \dot{X}^{\dot{B}} \dot{\partial}^{\dot{A}} \dot{Y}_{\dot{B}}-f^{\dot{A}}{ }_{\dot{B} \dot{C}} \dot{X}^{\dot{B}} \dot{Y}^{\dot{C}}
$$


Similarly, the right hand side of the equality in (3.39) reads

$$
\begin{aligned}
& \left(\left[\dot{\mathcal{L}}_{\dot{X}}, \dot{\mathcal{L}}_{\dot{Y}}\right]-\dot{\mathcal{L}}_{[\dot{X}, \dot{Y}]_{\mathrm{C}}}\right) \dot{T}_{\dot{A}_{1} \cdots \dot{A}_{n}} \\
& =\left(\frac{1}{2} \dot{X}^{\dot{N}} \dot{\partial}^{\dot{M}} \dot{Y}_{\dot{N}}-\frac{1}{2} \dot{Y}^{\dot{N}} \dot{\partial}^{\dot{M}} \dot{X}_{\dot{N}}+\Omega^{\dot{M}} \dot{N} \dot{G} \dot{X}^{\dot{N}} \dot{Y}^{\dot{G}}\right) \dot{\partial}_{\dot{M}} \dot{T}_{\dot{A}_{1} \cdots \dot{A}_{n}} \\
& +\frac{1}{2} \omega\left[\dot{X}^{\dot{N}} \dot{\partial}_{\dot{M}} \dot{\partial}^{\dot{M}} \dot{Y}_{\dot{N}}-\dot{Y} \dot{X}_{\dot{M}} \dot{\partial}^{\dot{M}} \dot{X}_{\dot{N}}+2 \dot{X}^{\dot{N}} \Omega_{\dot{N} \dot{G}}^{\dot{M}} \dot{\partial}_{\dot{M}} \dot{Y}^{\dot{G}}-2 \dot{Y}^{\dot{N}} \Omega_{\dot{N} \dot{G}}^{\dot{M}} \dot{\partial}_{\dot{M}} \dot{X}^{\dot{G}}\right. \\
& \left.+2 \dot{X} \dot{N}^{\dot{Y}} \dot{G} \dot{\partial}_{\dot{M}} \Omega_{\dot{N} \dot{G}}^{\dot{M}}+f_{\dot{M}}\left(\dot{X}^{\dot{N}} \dot{D}^{\dot{M}} \dot{Y}_{\dot{N}}-\dot{Y}^{\dot{N}} \dot{D}^{\dot{M}} \dot{X}_{\dot{N}}\right)\right] \dot{T}_{\dot{A}_{1} \cdots \dot{A}_{n}} \\
& +\sum_{i=1}^{n}\left[\dot{\partial}_{\dot{M}} \dot{Y}_{\dot{A}_{i}} \dot{\partial}^{\dot{M}} \dot{X}_{\dot{B}}-\dot{\partial}_{\dot{M}} \dot{X}_{\dot{A}_{i}} \dot{\partial}^{\dot{M}} \dot{Y}_{\dot{B}}+3 \Omega_{\dot{M}\left[\dot{A}_{i} \dot{B}\right.} \dot{X}^{\dot{N}} \dot{\partial}^{\dot{M}} \dot{Y}_{\dot{N}]}-3 \Omega_{\dot{M}\left[\dot{A}_{i} \dot{B}\right.} \dot{Y}^{\dot{N}} \dot{\partial}^{\dot{M}} \dot{X}_{\dot{N}]}\right. \\
& \left.\left.-\frac{1}{2} \Omega_{\dot{M} \dot{A}_{i} \dot{B}}\left(\dot{X}^{\dot{N}} \dot{\partial}^{\dot{M}} \dot{Y}_{\dot{N}}-\dot{Y}^{\dot{N}} \dot{\partial}^{\dot{M}} \dot{X}_{\dot{N}}\right)-3 \Omega_{\dot{M}[\dot{B} \dot{N}} \Omega^{\dot{M}} \dot{G}\right] \dot{A}_{i} \dot{X}^{\dot{N}} \dot{Y}^{\dot{G}}\right] \dot{T}_{\dot{A}_{1} \cdots \dot{A}_{i-1}} \dot{B}_{\dot{A}_{i+1} \cdots} \dot{A}_{n},
\end{aligned}
$$

which further becomes, using (3.31) and (3.34),

$$
\begin{aligned}
& \left(\left[\dot{\mathcal{L}}_{\dot{X}}, \dot{\mathcal{L}}_{\dot{Y}}\right]-\dot{\mathcal{L}}_{[\dot{X}, \dot{Y}]_{\mathrm{C}}}\right) \dot{T}_{\dot{A}_{1} \cdots \dot{A}_{n}} \\
& =\left(\frac{1}{2} \dot{X}^{\dot{N}} \dot{\partial}^{\dot{M}} \dot{Y}_{\dot{N}}-\frac{1}{2} \dot{Y}^{\dot{N}} \dot{\partial}^{\dot{M}} \dot{X}_{\dot{N}}+\Omega^{\dot{M}} \dot{N} \dot{G} \dot{X}^{\dot{N}} \dot{Y}^{\dot{G}}\right) \dot{\partial}_{\dot{M}} \dot{T}_{\dot{A}_{1} \cdots \dot{A}_{n}} \\
& +\frac{1}{2} \omega\left[\dot{X}^{\dot{N}} \dot{\partial}_{\dot{M}} \dot{\partial}^{\dot{M}} \dot{Y}_{\dot{N}}-\dot{Y}^{\dot{N}} \dot{\partial}_{\dot{M}} \dot{\partial}^{\dot{M}} \dot{X}_{\dot{N}}+2 \dot{X}^{\dot{N}} \Omega_{\dot{N} \dot{G}}^{\dot{M}} \dot{\partial}_{\dot{M}} \dot{Y}^{\dot{G}}-2 \dot{Y}{ }^{\dot{N}} \Omega_{\dot{N} \dot{G}} \dot{\partial}_{\dot{M}} \dot{X}^{\dot{G}}\right. \\
& \left.+2 \dot{X}^{N} \dot{Y}^{\dot{G}}\left(\dot{\partial}^{\dot{M}} f_{\dot{M} \dot{N} \dot{G}}+f^{\dot{M}} f_{\dot{M} \dot{N} \dot{G}}+2 \dot{\partial}_{[\dot{N}} f_{\dot{G}]}\right)+f_{\dot{M}}\left(\dot{X}^{\dot{N}^{\prime}} \dot{\partial}^{M} \dot{Y}_{\dot{N}}-\dot{Y}^{\dot{N}} \dot{\partial}^{M} \dot{X}_{\dot{N}}\right)\right] \dot{T}_{\dot{A}_{1} \cdots \dot{A}_{n}} \\
& +\sum_{i=1}^{n}\left[\dot{\partial}_{\dot{M}} \dot{Y}_{\dot{A}_{i}} \dot{\partial}^{\dot{M}} \dot{X}_{\dot{B}}-\dot{\partial}_{\dot{M}} \dot{X}_{\dot{A}_{i}} \dot{\partial}^{\dot{M}} \dot{Y}_{\dot{B}}-\frac{1}{2} \Omega_{\dot{M} \dot{A}_{i} \dot{B}}\left(\dot{X}^{\dot{N}} \dot{\partial}^{\dot{M}} \dot{Y}_{\dot{N}}-\dot{Y}^{\dot{N}} \dot{\partial}^{\dot{M}} \dot{X}_{\dot{N}}\right)\right. \\
& +3 \Omega_{\dot{M}\left[\dot{A}_{i} \dot{B}\right.} \dot{X}^{\dot{N}} \dot{\partial}^{\dot{M}} \dot{Y}_{\dot{N}]}-3 \Omega_{\dot{M}\left[\dot{A}_{i} \dot{B}\right.} \dot{Y}^{\dot{N}} \dot{\partial}^{\dot{M}} \dot{X}_{\dot{N}]} \\
& \left.\left.+\dot{X}^{\dot{N}} \dot{Y} \dot{G}\left(\dot{\partial}_{\dot{A}_{i}} f_{\dot{B} \dot{N} \dot{G}}-3 f_{\dot{M}[\dot{B} \dot{N}} f^{\dot{M}} \dot{G}\right] \dot{A}_{i}-3 \dot{\partial}_{[\dot{B}} f_{\dot{N} \dot{G}] \dot{A}_{i}}\right)\right] \dot{T}_{\dot{A}_{1} \cdots \dot{A}_{i-1}} \dot{B}_{\dot{A}_{i+1} \cdots \dot{A}_{n}} .
\end{aligned}
$$

Now we can easily read off a set of conditions, or the twistability conditions, which let each term in the right hand of the above equality vanish. The twistability conditions which ensure the closure of the U-twisted generalized Lie derivative

$$
\left[\dot{\mathcal{L}}_{\dot{X}}, \dot{\mathcal{L}}_{\dot{Y}}\right] \equiv \dot{\mathcal{L}}_{[\dot{X}, \dot{Y}]_{\mathrm{C}}}
$$

are as follows, cf. $[27,29] .{ }^{8}$

1. The section condition for all the dotted twisted fields,

$$
\dot{\partial}_{\dot{M}} \dot{\partial}^{\dot{M}} \equiv 0 \text {. }
$$

${ }^{8}$ Strictly speaking, our twistability conditions, especially (3.47), do not completely agree with the previous works. Yet, with the ansatz (3.51) assumed, they agree. 
2. The orthogonality between the connection and the derivatives of the dotted twisted fields,

$$
\Omega^{\dot{M}}{ }_{\dot{F} \dot{G}} \dot{\partial}_{\dot{M}} \equiv 0
$$

3. The Jacobi identity for $f_{\dot{A} \dot{B} \dot{C}}=f_{[\dot{A} \dot{B} \dot{C}]}$,

$$
f_{[\dot{A} \dot{B}} \dot{E} f_{\dot{C}] \dot{D} \dot{E}} \equiv 0 .
$$

4. The constancy of the structure constant, $f_{\dot{A} \dot{B} \dot{C}}$,

$$
\dot{\partial}_{\dot{E}} f_{\dot{A} \dot{B} \dot{C}} \equiv 0 \text {. }
$$

5. The triviality of $f_{\dot{A}}$,

$$
f_{\dot{A}}=\Omega_{\dot{C} \dot{A}}^{\dot{C}}-2 \dot{\partial}_{\dot{A}} \lambda=\partial_{C} U_{\dot{A}}^{C}-2 \dot{\partial}_{\dot{A}} \lambda \equiv 0 .
$$

We stress that these five constraints, (3.46)-(3.50), are the natural requirement for the closure (3.45) directly read off from (3.44). ${ }^{9}$ In principle, we should solve these constraints. While we are currently lacking the most general form of the solutions, a class of solutions are well known which involve dimensional reductions. If we assume the $U$ matrix to be in a block diagonal form,

$$
U=\left(\begin{array}{ll}
1 & 0 \\
0 & u
\end{array}\right),
$$

the dotted $\mathbf{O}(D, D)$ indices naturally split into effectively untwisted external indices and truly twisted internal indices. Letting all the twisted (or dotted) fields depend on the external coordinates while allowing the twisting data, $u, \lambda$, to have only the internal dependency, the first condition (3.46) is nothing but the ordinary section condition for the twisted fields living in the dimensionally reduced, external doubled-yet-gauged spacetime, and the second condition (3.47) is clearly satisfied. The remaining conditions (3.48), (3.49), (3.50) are then the genuine consistency conditions for the internal twisting data, $u$ and $\lambda$. This 'solution' then inevitably implies the dimensional reduction of the section, from $D=10$ to a lower value. Namely, the twistability conditions consist of the ordinary section condition for the external spacetime and a set of consistency conditions for the twisting data, $U$ and $\lambda$, of the orthogonal internal "manifold". It is interesting to explore other type of solution, if any, generalizing the ansatz (3.51). Anyhow, all the forthcoming analyses require strictly the five constraints, (3.46)-(3.50) only, and do not necessarily demand the ansatz (3.51).

It is worth while to note from (3.31), (3.34), that the twistability conditions imply

$$
\begin{aligned}
\dot{\partial}^{\dot{C}} \Omega_{\dot{C} \dot{A} \dot{B}} & \equiv 0, \\
\Omega_{\dot{E}[\dot{A} \dot{B}} \Omega_{\dot{C}] \dot{D}}^{\dot{E}} & \equiv 0, \\
\dot{\partial}_{\dot{A}} \dot{\partial}^{\dot{A}} \lambda \equiv \frac{1}{2} \dot{\partial}_{\dot{A}} \Omega_{\dot{B}} \dot{B} \dot{A} & =-\frac{1}{4} \Omega_{\dot{A}} \dot{A} \dot{C} \Omega^{\dot{B}}{ }_{\dot{B} \dot{C}}-\frac{1}{4} \Omega_{\dot{C} \dot{B} \dot{A}} \Omega^{\dot{B} \dot{C} \dot{A}} .
\end{aligned}
$$

\footnotetext{
${ }^{9}$ Clearly the five constraints, (3.46)-(3.50), are sufficient for the closure. It remains as an open question whether they are also necessary.
} 
Further, from the integrability of the last condition (3.50), we get

$$
\partial_{A}\left(U_{B} \dot{E} \partial_{C} U_{\dot{E}}^{C}\right) \equiv \partial_{B}\left(U_{A} \dot{E} \partial_{C} U_{\dot{E}}^{C}\right) .
$$

The U-twisted generalized Lie derivative (3.41) reduces, upon the twistability conditions, to

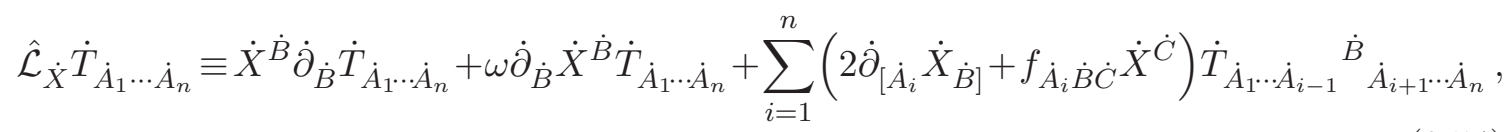

which clearly has no 'internal' coordinate dependency ${ }^{10}$ and decomposes into the external diffeomorphism and internal gauge symmetry $[27,29]$ (see also [50]).

\subsection{Twisted semi-covariant formalism}

The twisting of the semi-covariant formalism is straightforward. The U-twisted master semi-covariant derivative is

$$
\dot{\mathcal{D}}_{\dot{A}}=\dot{\nabla}_{\dot{A}}+\dot{\Phi}_{\dot{A}}+\dot{\bar{\Phi}}_{\dot{A}}
$$

of which the twisted semi-covariant derivative and the twisted spin connections are given by

$$
\dot{\nabla}_{\dot{A}}=\dot{D}_{\dot{A}}+\dot{\Gamma}_{\dot{A}}, \quad \dot{\Phi}_{\dot{A} p q}=\dot{V}_{p}^{\dot{B}} \dot{\nabla}_{\dot{A}} \dot{V}_{\dot{B} q}, \quad \dot{\bar{\Phi}}_{\dot{A} \bar{p} \bar{q}}=\dot{\bar{V}}_{\bar{p}}^{\dot{B}} \dot{\nabla}_{\dot{A}} \dot{\bar{V}}_{\dot{B} \bar{q}}
$$

and the twisted torsionless connection reads

$$
\begin{aligned}
\dot{\Gamma}_{\dot{C} \dot{A} \dot{B}}= & 2\left(\dot{P} \dot{D}_{\dot{C}} \dot{P} \dot{\bar{P}}\right)_{[\dot{A} \dot{B}]}+2\left(\dot{\bar{P}}_{[\dot{A}} \dot{D}_{\bar{P}} \dot{B}_{\dot{B}]}^{\dot{E}}-\dot{P}_{[\dot{A}} \dot{D}^{\dot{P}_{\dot{B}]}}{ }^{\dot{E}}\right) \dot{D}_{\dot{D}} \dot{P}_{\dot{E} \dot{C}} \\
& -\frac{4}{D-1}\left(\dot{\bar{P}}_{\dot{C}[\dot{A}} \dot{\bar{P}}_{\dot{B}]} \dot{D}+\dot{P}_{\dot{C}[\dot{A}} \dot{P}_{\dot{B}]} \dot{D}\right)\left(\dot{D}_{\dot{D}} \dot{d}+\left(\dot{P} \dot{D}^{\dot{E}} \dot{P} \dot{\bar{P}}\right)_{[\dot{E} \dot{D}]}\right),
\end{aligned}
$$

satisfying, in a completely parallel manner to the untwisted cases, (2.21)-(2.25),

$$
\begin{aligned}
& \dot{\nabla}_{\dot{A}} \dot{P}_{\dot{B} \dot{C}}=0, \quad \dot{\nabla}_{\dot{A}} \dot{\bar{P}}_{\dot{B} \dot{C}}=0, \quad \dot{\Gamma}^{\dot{B}}{ }_{\dot{B} \dot{A}}=-2 \dot{D}_{\dot{A}} \dot{d}, \\
& \dot{\Gamma}_{\dot{A}(\dot{B} \dot{C})}=0, \quad \dot{\Gamma}_{[\dot{A} \dot{B} \dot{C}]}=0, \quad(\dot{\mathcal{P}}+\dot{\overline{\mathcal{P}}})_{\dot{A} \dot{B} \dot{C}} \dot{D} \dot{E} \dot{F} \dot{\Gamma}_{\dot{D} \dot{E} \dot{F}}=0,
\end{aligned}
$$

and, as for the torsionless condition (2.26),

$$
\hat{\mathcal{L}}_{X}(\dot{D})=\hat{\mathcal{L}}_{X}(\dot{\nabla}), \quad[X, Y]_{\mathrm{C}}(\dot{D})=[X, Y]_{\mathrm{C}}(\dot{\nabla}) .
$$

Further, from (2.42), (2.47), in terms of

$$
\begin{aligned}
\dot{\mathcal{F}}_{\dot{A} \dot{B} p q} & =\dot{\nabla}_{\dot{A}} \dot{\Phi}_{\dot{B} p q}-\dot{\nabla}_{\dot{B}} \dot{\Phi}_{\dot{A} p q}+\dot{\Phi}_{\dot{A} p}{ }^{r} \dot{\Phi}_{\dot{B} r q}-\dot{\Phi}_{\dot{B} p}{ }^{r} \dot{\Phi}_{\dot{A} r q} \\
& =\dot{\mathcal{D}}_{\dot{A}} \dot{\Phi}_{\dot{B} p q}-\dot{\mathcal{D}}_{\dot{B}} \dot{\Phi}_{\dot{A} p q}-\dot{\Phi}_{\dot{A} p}{ }^{r} \dot{\Phi}_{\dot{B} r q}+\dot{\Phi}_{\dot{B} p}{ }^{r} \dot{\Phi}_{\dot{A} r q}, \\
\dot{\overline{\mathcal{F}}}_{\dot{A} \dot{B} \bar{p} \bar{q}} & =\dot{\nabla}_{\dot{A}} \dot{\bar{\Phi}}_{\dot{B} \bar{p} \bar{q}}-\dot{\nabla}_{\dot{B}} \dot{\bar{\Phi}}_{\dot{A} \bar{p} \bar{q}}+\dot{\bar{\Phi}}_{\dot{A} \bar{p}} \bar{r} \dot{\Phi}_{\dot{B} \bar{r} \bar{q}}-\dot{\bar{\Phi}}_{\dot{B} \bar{p}}{ }^{r} \dot{\Phi}_{\dot{A} \bar{r} \bar{q}} \\
& =\dot{\mathcal{D}}_{\dot{A}} \dot{\bar{\Phi}}_{\dot{B} \bar{p} \bar{q}}-\dot{\mathcal{D}}_{\dot{B}} \dot{\bar{\Phi}}_{\dot{A} \bar{p} \bar{q}}-\dot{\bar{\Phi}}_{\dot{A} \bar{p}}{ }^{r} \dot{\Phi}_{\dot{B} \bar{r} \bar{q}}+\dot{\bar{\Phi}}_{\dot{B} \bar{p}}{ }^{r} \dot{\Phi}_{\dot{A} \bar{r} \bar{q}},
\end{aligned}
$$

\footnotetext{
${ }^{10}$ With the internal/external splitting (3.51), the $\dot{\partial}_{\dot{A}}$ derivatives of the dotted fields are independent of the internal coordinates.
} 
we have the twisted spinorial semi-covariant four-index curvature,

$$
\dot{\mathcal{G}}_{\dot{A} \dot{B} \dot{C} \dot{D}}:=\frac{1}{2}\left[(\dot{\mathcal{F}}+\dot{\overline{\mathcal{F}}})_{\dot{A} \dot{B} \dot{C} \dot{D}}+(\dot{\mathcal{F}}+\dot{\overline{\mathcal{F}}})_{\dot{C} \dot{D} \dot{A} \dot{B}}+(\dot{\Phi}+\dot{\bar{\Phi}})^{\dot{E}}{ }_{\dot{A} \dot{B}}(\dot{\Phi}+\dot{\bar{\Phi}})_{\dot{E} \dot{C} \dot{D}}\right] .
$$

Now, from (2.49), it is useful to note

$$
\dot{\mathcal{G}}_{\dot{A} \dot{B} \dot{C} \dot{D}}=\dot{S}_{\dot{A} \dot{B} \dot{C} \dot{D}}+\frac{1}{2}\left(\dot{V}_{\dot{A}}^{p} \dot{D}_{\dot{E}} \dot{V}_{\dot{B} p}+\dot{\bar{V}}_{\dot{A}}^{\bar{p}} \dot{D}_{\dot{E}} \dot{\bar{V}}_{\dot{B} \bar{p}}\right)\left(\dot{V}_{\dot{C}}^{q} \dot{D}^{\dot{E}^{E}} \dot{V}_{\dot{D} q}+\dot{\bar{V}}_{\dot{C}}^{\bar{q}} \dot{D}^{\dot{E}} \dot{\bar{V}}_{\dot{D} \bar{q}}\right)
$$

and thus, upon the twistability conditions,

$$
\dot{\mathcal{G}}_{\dot{A} \dot{B} \dot{C} \dot{D}} \equiv \dot{S}_{\dot{A} \dot{B} \dot{C} \dot{D}}+\frac{1}{2} \Omega_{\dot{E} \dot{A} \dot{B}} \Omega^{\dot{E}} \dot{C} \dot{D}
$$

In the above, for sure, we set

$$
\begin{aligned}
& \dot{S}_{\dot{A} \dot{B} \dot{C} \dot{D}}=\frac{1}{2}\left(\dot{R}_{\dot{A} \dot{B} \dot{C} \dot{D}}+\dot{R}_{\dot{C} \dot{D} \dot{A} \dot{B}}-\dot{\Gamma}^{\dot{E}}{ }_{\dot{A} \dot{B}} \dot{\Gamma}_{\dot{E} \dot{C} \dot{D}}\right), \\
& \dot{R}_{\dot{A} \dot{B} \dot{C} \dot{D}}=\dot{\partial}_{\dot{A}} \dot{\Gamma}_{\dot{B} \dot{C} \dot{D}}-\dot{\partial}_{\dot{B}} \dot{\Gamma}_{\dot{A} \dot{C} \dot{D}}+\dot{\Gamma}_{\dot{A} \dot{C}} \dot{E}_{\dot{\Gamma}_{\dot{B} \dot{E} \dot{D}}}-\dot{\Gamma}_{\dot{B} \dot{C}} \dot{\Gamma}_{\dot{A} \dot{E} \dot{D}}
\end{aligned}
$$

Thus, in contrast to the untwisted case (2.50), $\mathcal{G}_{\dot{A} \dot{B} \dot{C} \dot{D}}$ differs from $\dot{S}_{\dot{A} \dot{B} \dot{C} \dot{D}}$ after the twist. In the twisted SDFT to be constructed below, we shall disregard the latter and employ the former only. The former will be shown to be semi-covariant, while the latter is not.

Starting from the strict equality of (3.62) and using (2.52), one can easily show nevertheless that the infinitesimal transformation of $\dot{\mathcal{G}} \dot{A} \dot{B} \dot{C} \dot{D}$ induced by the variations of its constituting all the twisted fields coincides with that of $\dot{S}_{\dot{A} \dot{B} \dot{C} \dot{D}}$, up to the twistability conditions,

$$
\delta \dot{\mathcal{G}}_{\dot{A} \dot{B} \dot{C} \dot{D}} \equiv \dot{\nabla}_{[\dot{A}} \delta \dot{\Gamma}_{\dot{B}] \dot{C} \dot{D}}+\dot{\nabla}_{[\dot{C}} \delta \dot{\Gamma}_{\dot{D}] \dot{A} \dot{B}} \equiv \delta \dot{S}_{\dot{A} \dot{B} \dot{C} \dot{D}}
$$

This should be a naturally expected result, if we focus on the variation of the equivalence relation (3.63) rather than the strict equality (3.62). Since $\Omega_{\dot{A} \dot{B} \dot{C}}$ is not a field variable but rather a fixed data for a given internal manifold, it is not taken to transform but must be inert under any 'symmetry', 11

$$
\delta U_{A} \dot{B}=0, \quad \delta \Omega_{\dot{C} \dot{A} \dot{B}}=0, \quad \delta\left(\dot{D}_{\dot{C}}\right)=0 .
$$

These are also consistent with (3.4) and (3.38) with the identification of ' $\delta_{\dot{X}}=\dot{L}_{\dot{X}}$ ' for covariant twisted fields.

- Complete covariantizations after the twist.

Here we focus on the twisted diffeomorphism. We twist the relation (2.57) in order to obtain the difference between the actual transformation of the U-derivative of a twisted field and its twisted generalized Lie derivative,

$$
\begin{aligned}
& \left(\delta_{\dot{X}}-\dot{\mathcal{L}}_{\dot{X}}\right) \dot{D}_{\dot{C}} \dot{T}_{\dot{A}_{1} \cdots \dot{A}_{n}} \\
& \quad=\left[\dot{D}_{\dot{C}}, \dot{\mathcal{L}}_{\dot{X}}\right] \dot{T}_{\dot{A}_{1} \cdots \dot{A}_{n}} \\
& \quad=\dot{D}^{\dot{B}} \dot{X}_{\dot{C}} \dot{D}_{\dot{B}} \dot{T}_{\dot{A}_{1} \cdots \dot{A}_{n}}+\omega_{T} \dot{D}_{\dot{C}} \dot{D}_{\dot{B}} \dot{X}^{\dot{B}} \dot{T}_{\dot{A}_{1} \cdots \dot{A}_{n}}+\sum_{i=1}^{n} 2 \dot{D}_{\dot{C}} \dot{D}_{\left[\dot{A}_{i}\right.} \dot{X}_{\dot{B}]} \dot{T}_{\dot{A}_{1} \cdots \dot{A}_{i-1}} \dot{B}_{i+1} \cdots \dot{A}_{n}
\end{aligned}
$$

\footnotetext{
${ }^{11}$ However, $\hat{\mathcal{L}}_{\dot{X}} U_{A}{ }^{\dot{B}}=\dot{X}^{\dot{C}} \dot{D}_{\dot{C}} U_{A}{ }^{\dot{B}}+\left(\dot{D}^{\dot{B}} \dot{X}_{\dot{C}}-\dot{D}_{\dot{C}} \dot{X}^{\dot{B}}\right) U_{A}{ }^{\dot{C}}=\dot{D}^{\dot{B}} X_{A}-D_{A} \dot{X}^{\dot{B}} \neq 0$.
} 
Writing the first equality above, we have implicitly assumed (3.66). It follows for the twisted connection (3.57),

$$
\begin{aligned}
\left(\delta_{\dot{X}}-\hat{\mathcal{L}}_{\dot{X}}\right) \dot{\Gamma}_{\dot{C} \dot{A} \dot{B}}= & 2\left[(\dot{\mathcal{P}}+\dot{\overline{\mathcal{P}}})_{\dot{C} \dot{A} \dot{B}} \dot{F} \dot{D} \dot{E}-\delta_{\dot{C}}^{\dot{F}} \delta_{\dot{A}}^{\dot{D}} \delta_{\dot{B}}^{\dot{E}}\right] \dot{D}_{\dot{F}} \dot{D}_{[\dot{D}} \dot{X}_{\dot{E}]} \\
& +2\left(\dot{\mathcal{P}}^{\prime}-\dot{\mathcal{P}}^{\prime}\right)_{\dot{C} \dot{A} \dot{B}} \dot{F} \dot{D} \dot{E} \dot{D}^{\dot{G}} \dot{P}_{\dot{F} \dot{D}} \dot{D}_{\dot{G}} \dot{X}_{\dot{E}}+2 \dot{P}_{[\dot{A}} \dot{D} \dot{\bar{P}}_{\dot{B}]} \dot{E}^{E} \dot{D}^{\dot{G}} \dot{P}_{\dot{D} \dot{E}} \dot{D}_{\dot{G}} \dot{X}_{\dot{C}} \\
& +\frac{2}{D-1}\left(\dot{P}_{\dot{C}[\dot{A}} \dot{P}_{\dot{B}]} \dot{E}+\dot{\bar{P}}_{\dot{C}[\dot{A}} \dot{\bar{P}}_{\dot{B}]} \dot{E}^{\dot{E}}\right)\left(\dot{D}_{\dot{G}} \dot{D}^{\dot{G}} \dot{X}_{\dot{E}}-2 \dot{D}^{\dot{G}} \dot{d} \dot{D}_{\dot{G}} \dot{X}_{\dot{E}}\right)
\end{aligned}
$$

To simplify this expression up to the twistability conditions, we use (3.31) and an identity,

$$
\begin{aligned}
& {\left[2\left(\dot{\mathcal{P}}^{\prime}+\dot{\overline{\mathcal{P}}}^{\prime}\right)_{\dot{C} \dot{A} \dot{B}} \dot{F} \dot{D} \dot{E}+2 \dot{P}_{[\dot{A}} \dot{D} \dot{\bar{P}}_{\dot{B}]} \dot{F}_{\dot{C}}^{\dot{E}}+\frac{2}{D-1}\left(\dot{P}_{\dot{C}[\dot{A}} \dot{P}_{\dot{B}]} \dot{F}+\dot{\bar{P}}_{\dot{C}[\dot{A}} \dot{\bar{P}}_{\dot{B}]} \dot{F}\right) \dot{\mathcal{J}} \dot{D} \dot{E}\right] \Omega_{\dot{G} \dot{F} \dot{D}} \Omega_{\dot{E} \dot{K}} \dot{K}} \\
& \left.=3\left(\dot{P}_{\dot{C}} \dot{F} \dot{\bar{P}}_{\dot{A}} \dot{D} \dot{\bar{P}}_{\dot{B}}^{\dot{E}}+\dot{\bar{P}}_{\dot{C}} \dot{F} \dot{P}_{\dot{A}}^{\dot{D}} \dot{P}_{\dot{B}} \dot{E}\right) \Omega_{\dot{G}\left[\dot{D} \dot{E} \Omega^{\dot{G}}\right.} \dot{F}\right] \dot{K} \\
& +\left[(\dot{\mathcal{P}}+\dot{\overline{\mathcal{P}}})_{\dot{C} \dot{A} \dot{B}} \dot{F} \dot{D} \dot{E}-\delta_{\dot{C}}^{\dot{F}} \delta_{\dot{A}}^{\dot{D}} \delta_{\dot{B}}^{\dot{E}}\right] \Omega_{\dot{G} \dot{D} \dot{E}} \Omega^{\dot{G}} \dot{F} \dot{K} \\
& =3\left(\dot{P}_{\dot{C}} \dot{F} \dot{\bar{P}}_{\dot{A}} \dot{D} \dot{\bar{P}}_{\dot{B}}^{\dot{E}}+\dot{\bar{P}}_{\dot{C}} \dot{F} \dot{P}_{\dot{A}} \dot{D}^{\dot{P}_{\dot{B}}} \dot{E}\right)\left(f_{[\dot{D} \dot{E}} \dot{G} f_{\dot{F}] \dot{K} \dot{G}}+\dot{\partial}_{[\dot{D}} f_{\dot{E} \dot{F}] \dot{K}}-\frac{1}{3} \dot{\partial}_{\dot{K}} f_{\dot{D} \dot{E} \dot{F}}\right) \\
& +\left[(\dot{\mathcal{P}}+\dot{\overline{\mathcal{P}}})_{\dot{C} \dot{A} \dot{B}} \dot{F} \dot{D} \dot{E}-\delta_{\dot{C}} \dot{F} \delta_{\dot{A}} \dot{D} \delta_{\dot{B}}^{\dot{E}}\right] \Omega_{\dot{G} \dot{D} \dot{E}} \Omega_{\dot{F} \dot{K}}^{\dot{K}},
\end{aligned}
$$

which follows from (3.34). Eq. (3.68) can be then rewritten as

$$
\begin{aligned}
& \left(\delta_{\dot{X}}-\hat{\mathcal{L}}_{\dot{X}}\right) \dot{\Gamma}_{\dot{C} \dot{A} \dot{B}} \\
& =\left[(\dot{\mathcal{P}}+\dot{\overline{\mathcal{P}}})_{\dot{C} \dot{A} \dot{B}} \dot{F} \dot{D} \dot{E}-\delta_{\dot{C}}^{\dot{F}} \delta_{\dot{A}}^{\dot{D}} \delta_{\dot{B}}^{\dot{E}}\right]\left(2 \dot{D}_{\dot{F}} \dot{D}_{[\dot{D}} \dot{X}_{\dot{E}]}+\Omega_{\dot{G} \dot{D} \dot{E}} \Omega^{\dot{G}} \dot{F} \dot{K} \dot{X}^{\dot{K}}\right) \\
& +2\left(\dot{\mathcal{P}}^{\prime}-\dot{\overline{\mathcal{P}}}^{\prime}\right)_{\dot{C} \dot{A} \dot{B}} \dot{F} \dot{D} \dot{E}\left[\left(\dot{\partial}_{\dot{G}} \dot{P}_{\dot{F} \dot{D}}+\Omega_{\dot{G} \dot{F}} \dot{K} \dot{P}_{\dot{K} \dot{D}}+\Omega_{\dot{G} \dot{D}} \dot{K}_{\dot{P}} \dot{P}_{\dot{F}}\right) \dot{\partial}^{\dot{G}^{\prime}} \dot{X}_{\dot{E}}+\dot{\partial}_{\dot{G}} \dot{P}_{\dot{F} \dot{D}} \Omega^{\dot{G}} \dot{E} \dot{K} \dot{X}^{\dot{K}}\right] \\
& +2 \dot{P}_{[\dot{A}} \dot{D} \dot{\bar{P}}_{\dot{B}]}^{\dot{E}}\left[\left(\dot{\partial}_{\dot{G}} \dot{P}_{\dot{D} \dot{E}}+\Omega_{\dot{G} \dot{D}} \dot{K}_{\dot{P}} \dot{P}_{\dot{K}}+\Omega_{\dot{G} \dot{E}} \dot{K} \dot{P}_{\dot{D} \dot{K}}\right) \dot{\partial}^{\dot{G}} \dot{X}_{\dot{C}}+\dot{\partial}_{\dot{G}} \dot{P}_{\dot{D} \dot{E}} \Omega^{\dot{G}} \dot{C} \dot{K} \dot{X}^{\dot{K}}\right] \\
& +3\left(\dot{P}_{\dot{C}} \dot{F} \dot{\bar{P}}_{\dot{A}} \dot{D} \dot{\bar{P}}_{\dot{B}}^{\dot{E}}+\dot{\bar{P}}_{\dot{C}} \dot{F}^{P_{\dot{A}}} \dot{D}^{\dot{P}_{\dot{B}}} \dot{E}\right)\left(f_{[\dot{D E} \dot{E}} f_{\dot{F}] \dot{K} \dot{G}}+\dot{\partial}_{[\dot{D}} f_{\dot{E} \dot{F}] \dot{K}}-\frac{1}{3} \dot{\partial}_{\dot{K}} f_{\dot{D} \dot{E} \dot{F}}\right) \dot{X}^{\dot{K}} \\
& +\frac{2}{D-1}\left(\dot{P}_{\dot{C}[\dot{A}} \dot{P}_{\dot{B}]} \dot{E}+\dot{\bar{P}}_{\dot{C}[\dot{A}} \dot{\bar{P}}_{\dot{B}]} \dot{E}^{E}\right)\left[\dot{\partial}_{\dot{G}} \dot{\partial}^{\dot{G}} \dot{X}_{\dot{E}}+2 \Omega_{\dot{G} \dot{E} \dot{K}} \dot{\partial}^{\dot{G}} \dot{X}^{\dot{K}}\right. \\
& \left.+\left(f_{\dot{G}}-2 \dot{\partial}_{\dot{G}} \dot{d}\right)\left(\dot{\partial} \dot{G} \dot{X}_{\dot{E}}+\Omega^{\dot{G}} \dot{E}_{\dot{K}} \dot{X}^{\dot{K}}\right)\right] \\
& +\frac{2}{D-1}\left(\dot{P}_{\dot{C}[\dot{A}} \dot{P}_{\dot{B}]} \dot{E}+\dot{\bar{P}}_{\dot{C}[\dot{A}} \dot{\bar{P}}_{\dot{B}]}^{\dot{E}}\right)\left(\dot{\partial}^{\dot{F}} f_{\dot{F} \dot{E} \dot{K}}+2 \dot{\partial}_{[\dot{E}} f_{\dot{K}]}+2 f^{\dot{F}} \Omega_{[\dot{E} \dot{K}] \dot{F}}\right) \dot{X}^{\dot{K}}
\end{aligned}
$$

Hence, upon the twisted section conditions (3.46)-(3.50), we have a rather simple seminal expression,

$$
\left(\delta_{\dot{X}}-\hat{\mathcal{L}}_{\dot{X}}\right) \dot{\Gamma}_{\dot{C} \dot{A} \dot{B}} \equiv\left[(\dot{\mathcal{P}}+\dot{\overline{\mathcal{P}}})_{\dot{C} \dot{A} \dot{B}} \dot{F} \dot{D} \dot{E}-\delta_{\dot{C}} \dot{F} \delta_{\dot{A}} \dot{D}_{\dot{B}}^{\dot{E}}\right]\left(2 \dot{D}_{\dot{F}} \dot{D}_{[\dot{D}} \dot{X}_{\dot{E}]}+\Omega_{\dot{G} \dot{D} \dot{E}} \Omega^{\dot{G}} \dot{F} \dot{K} \dot{X}^{\dot{K}}\right) .
$$


From this, the diffeomorphic anomaly of the twisted semi-covariant derivative follows easily,

$$
\begin{aligned}
& \left(\delta_{\dot{X}}-\hat{\mathcal{L}}_{\dot{X}}\right)\left(\dot{\nabla}_{\dot{C}} \dot{T}_{\dot{A}_{1} \cdots \dot{A}_{n}}\right) \\
& =\dot{D}^{\dot{E}} \dot{X}_{\dot{C}} \dot{D}_{\dot{E}} \dot{T}_{\dot{A}_{1} \cdots \dot{A}_{n}}+\omega_{T}\left[\dot{D}_{C} \dot{D}_{\dot{E}} \dot{X}^{\dot{E}}-\left(\delta_{\dot{X}}-\hat{\mathcal{L}}_{\dot{X}}\right) \dot{\Gamma}^{\dot{E}}{ }_{\dot{E} \dot{C}}\right] \dot{T}_{\dot{A}_{1} \cdots \dot{A}_{n}} \\
& +\sum_{i=1}^{n}\left[2 \dot{D}_{\dot{C}} \dot{D}_{\left[\dot{A}_{i}\right.} X_{\dot{E}]}+\left(\delta_{\dot{X}}-\hat{\mathcal{L}}_{\dot{X}}\right) \dot{\Gamma}_{\dot{C} \dot{A}_{i} \dot{E}}\right] \dot{T}_{\dot{A}_{1} \cdots \dot{A}_{i-1}} \dot{E}_{\dot{A}_{i+1} \cdots \dot{A}_{n}} \\
& \equiv \omega_{T} f_{\dot{K}} \dot{D}^{\dot{K}} \dot{X}_{\dot{C}} \dot{T}_{\dot{A}_{1} \cdots \dot{A}_{n}} \\
& +\sum_{i=1}^{n}(\mathcal{P}+\overline{\mathcal{P}})_{\dot{C} \dot{A}_{i}} \dot{B} \dot{F} \dot{D} \dot{E}\left(2 \dot{D}_{\dot{F}} \dot{D}_{[\dot{D}} \dot{X}_{\dot{E}]}+\Omega_{\dot{G} \dot{D} \dot{E}} \Omega_{\dot{F} \dot{K}}^{\dot{K}} \dot{X}^{\dot{K}}\right) \dot{T}_{\ldots \dot{A}_{i-1} \dot{B} \dot{A}_{i+1} \ldots} .
\end{aligned}
$$

Hence, upon all the twistability conditions, finally we obtain

$$
\left(\delta_{\dot{X}}-\hat{\mathcal{L}}_{\dot{X}}\right)\left(\dot{\nabla}_{\dot{C}^{T}} \dot{T}_{\dot{A}_{1} \cdots \dot{A}_{n}}\right) \equiv \sum_{i=1}^{n}(\mathcal{P}+\overline{\mathcal{P}})_{\dot{C} \dot{A}_{i}} \dot{B}_{\dot{A}_{1} \cdots \dot{A}_{i-1} \dot{B} \dot{A}_{i+1} \cdots \dot{A}_{n}},
$$

where we have introduced shorthand notations,

$$
\begin{aligned}
& \dot{\mathcal{P}}_{\dot{A} \dot{B} \dot{C}}=\dot{\mathcal{P}}_{\dot{A} \dot{B} \dot{C}} \dot{F} \dot{D} \dot{E}\left(2 \dot{D}_{\dot{F}} \dot{D}_{[\dot{D}} \dot{X}_{\dot{E}]}+\Omega_{\dot{G} \dot{D} \dot{E}} \Omega^{\dot{G}}{ }_{\dot{F} \dot{K}} \dot{X}^{\dot{K}}\right), \\
& \dot{\overline{\mathcal{P}}}_{\dot{A} \dot{B} \dot{C}}=\dot{\overline{\mathcal{P}}}_{\dot{A} \dot{B} \dot{C}} \dot{F} \dot{D} \dot{E}\left(2 \dot{D}_{\dot{F}} \dot{D}_{[\dot{D}} \dot{X}_{\dot{E}]}+\Omega_{\dot{G} \dot{D} \dot{E}} \Omega^{\dot{G}}{ }_{\dot{F} \dot{K}} \dot{X}^{\dot{K}}\right)
\end{aligned}
$$

From (2.16) and (3.34), they satisfy up to the twistability conditions,

$$
\dot{\mathcal{P}}_{[\dot{A} \dot{B} \dot{C}]} \equiv 0, \quad \dot{\overline{\mathcal{P}}}_{[\dot{A} \dot{B} \dot{C}]} \equiv 0
$$

Eq. (3.73) immediately implies for the spin connections,

$$
\begin{aligned}
& \left(\delta_{\dot{X}}-\hat{\mathcal{L}}_{\dot{X}}\right) \dot{\Phi}_{\dot{A} p q}=\left(\delta_{\dot{X}}-\hat{\mathcal{L}}_{\dot{X}}\right)\left(\dot{V}^{\dot{B}}{ }_{p} \dot{\nabla}_{\dot{A}} \dot{V}_{\dot{B} q}\right) \equiv \dot{\mathcal{P}}_{\dot{A} p q}, \\
& \left(\delta_{\dot{X}}-\hat{\mathcal{L}}_{\dot{X}}\right) \dot{\bar{\Phi}}_{\dot{A} \bar{p} \bar{q}}=\left(\delta_{\dot{X}}-\hat{\mathcal{L}}_{\dot{X}}\right)\left(\dot{\bar{V}}^{\dot{B}} \dot{\bar{p}}_{\dot{\bar{p}}} \dot{\bar{V}}_{\dot{B} \bar{q}}\right) \equiv \dot{\overline{\mathcal{P}}}_{\dot{A} \bar{p} \bar{q}} .
\end{aligned}
$$

Although the final expressions of (3.73) and (3.76) differ in detail from what one would naively expect by 'twisting' the results of (2.65) and (2.85), ${ }^{12}$ what remains still true and crucial is that, once again the anomalies are all controlled by the index-six projection operators. Namely, they are still semi-covariant. Thus, the cancellation mechanism is identical before and after the twist, and all the previous completely covariant derivatives, (2.67), (2.68), (2.81) and (2.87) are still completely covariant

\footnotetext{
${ }^{12}$ Twisting (2.65) or (2.85) is naive, because they are not exact formulas. They are valid only up to the original section condition.
} 
after the twist. We recall them exhaustively after performing the twist,

$$
\begin{aligned}
& \dot{P}_{\dot{C}} \dot{D}_{\bar{P}}^{\dot{P}_{1}} \dot{B}_{1} \ldots \dot{\bar{P}}_{\dot{A}_{n}} \dot{B}_{n} \dot{\nabla}_{\dot{D}} \dot{T}_{\dot{B}_{1} \cdots \dot{B}_{n}}, \quad \quad \dot{\bar{P}}_{\dot{C}} \dot{D}^{\dot{D}} \dot{P}_{\dot{A}_{1}} \dot{B}_{1} \ldots \dot{P}_{\dot{A}_{n}} \dot{B}_{n} \dot{\nabla}_{\dot{D}} \dot{T}_{\dot{B}_{1} \cdots \dot{B}_{n}}, \\
& \dot{P}^{\dot{A} \dot{B}} \bar{P}_{\dot{C}_{1}} \dot{D}_{1} \ldots \dot{\bar{P}}_{\dot{C}_{n}} \dot{D}_{n} \dot{\nabla}_{\dot{A}} \dot{T}_{\dot{B} \dot{D}_{1} \cdots \dot{D}_{n}}, \quad \dot{\bar{P}}^{\dot{A} \dot{B}} \dot{P}_{\dot{C}_{1}} \dot{D}_{1} \ldots \dot{P}_{\dot{C}_{n}} \dot{D}_{n} \dot{\nabla}_{\dot{A}} \dot{T}_{\dot{B} \dot{D}_{1} \cdots \dot{D}_{n}}
\end{aligned}
$$

(divergences),

$\dot{P}^{A B} \dot{\bar{P}}_{\dot{C}_{1}} \dot{D}_{1} \ldots \dot{\bar{P}}_{\dot{C}_{n}} \dot{D}_{n} \dot{\nabla}_{\dot{A}} \dot{\nabla}_{\dot{B}} \dot{T}_{\dot{D}_{1} \ldots \dot{D}_{n}}, \quad \dot{\bar{P}}^{\dot{A} \dot{B}} \dot{P}_{\dot{C}_{1}} \dot{D}_{1} \ldots \dot{P}_{\dot{C}_{n}} \dot{D}_{n} \dot{\nabla}_{\dot{A}} \dot{\nabla}_{\dot{B}} \dot{T}_{\dot{D}_{1} \cdots \dot{D}_{n}}$

(Laplacians),

$\dot{\mathcal{D}}_{p} T_{\bar{q}_{1} \cdots \bar{q}_{n}}, \quad \dot{\mathcal{D}}_{\bar{p}} T_{q_{1} \cdots q_{n}}, \quad \dot{\mathcal{D}}_{p} T^{p} \bar{q}_{1} \cdots \bar{q}_{n}, \quad \dot{\mathcal{D}}_{\bar{p}} T^{\bar{p}} q_{1} \cdots q_{n}, \quad \dot{\mathcal{D}}_{p} \dot{\mathcal{D}}^{p} T_{\bar{q}_{1} \cdots \bar{q}_{n}}, \quad \dot{\mathcal{D}}_{\bar{p}} \dot{\mathcal{D}}^{\bar{p}} T_{q_{1} \cdots q_{n}}$,

$\dot{\mathcal{D}}_{+} \mathcal{T}, \quad \dot{\mathcal{D}}_{-} \mathcal{T}, \quad \dot{\mathcal{F}}=\dot{\mathcal{D}}_{+} \mathcal{C}$,

and

$$
\begin{aligned}
& \gamma^{p} \dot{\mathcal{D}}_{p} \rho, \quad \gamma^{p} \dot{\mathcal{D}}_{p} \psi_{\bar{p}}, \quad \dot{\mathcal{D}}_{\bar{p}} \rho, \quad \dot{\mathcal{D}}_{\bar{p}} \psi^{\bar{p}}, \quad \bar{\psi}^{\dot{A}} \gamma_{p}\left(\dot{\mathcal{D}}_{\dot{A}} \psi_{\bar{q}}-\frac{1}{2} \dot{\mathcal{D}}_{\bar{q}} \psi_{\dot{A}}\right) \\
& \bar{\gamma}^{\bar{p}} \dot{\mathcal{D}}_{\bar{p}} \rho^{\prime}, \quad \bar{\gamma}^{\bar{p}} \dot{\mathcal{D}}_{\bar{p}} \psi_{p}^{\prime}, \quad \dot{\mathcal{D}}_{p} \rho^{\prime}, \quad \dot{\mathcal{D}}_{p} \psi^{\prime p}, \quad \bar{\psi}^{\prime} \dot{\bar{\gamma}}_{\bar{p}}\left(\dot{\mathcal{D}}_{\dot{A}} \psi_{q}^{\prime}-\frac{1}{2} \dot{\mathcal{D}}_{q} \psi_{\dot{A}}^{\prime}\right) .
\end{aligned}
$$

Now we turn to the curvatures. The relations, (3.75), (3.76), give sequently,

$$
\begin{aligned}
&\left(\delta_{\dot{X}}-\hat{\mathcal{L}}_{\dot{X}}\right) \dot{\mathcal{F}}_{\dot{A} \dot{B} p q} \equiv 2 \dot{\mathcal{D}}_{[\dot{A}} \dot{\mathcal{P}}_{\dot{B}] p q}-(\dot{\mathcal{P}}+\dot{\overline{\mathcal{P}}})^{\dot{E}}{ }_{\dot{A} \dot{B}} \dot{\Phi}_{\dot{E} p q} \\
&\left(\delta_{\dot{X}}-\hat{\mathcal{L}}_{\dot{X}}\right) \dot{\mathcal{F}}_{\dot{A} \dot{B} \bar{p} \bar{q}} \equiv 2 \dot{\mathcal{D}}_{[\dot{A}} \dot{\overline{\mathcal{P}}}{ }_{\dot{B}] \bar{p} \bar{q}}-(\dot{\mathcal{P}}+\dot{\overline{\mathcal{P}}})^{\dot{E}}{ }_{\dot{A} \dot{B}} \dot{\bar{\Phi}}_{\dot{E} \bar{p} \bar{q}} \\
&\left(\delta_{\dot{X}}-\hat{\mathcal{L}}_{\dot{X}}\right)(\dot{\mathcal{F}}+\dot{\overline{\mathcal{F}}})_{\dot{A} \dot{B} \dot{C} \dot{D}} \equiv 2 \dot{\mathcal{D}}_{[\dot{A}}(\dot{\mathcal{P}}+\dot{\overline{\mathcal{P}}})_{\dot{B}] \dot{C} \dot{D}}-(\dot{\mathcal{P}}+\dot{\overline{\mathcal{P}}})^{\dot{E}}{ }_{\dot{A} \dot{B}}(\dot{\Phi}+\dot{\bar{\Phi}})_{\dot{E} \dot{C} \dot{D}}
\end{aligned}
$$

and thus, another crucial result follows

$$
\left(\delta_{\dot{X}}-\hat{\mathcal{L}}_{\dot{X}}\right) \dot{\mathcal{G}}_{\dot{A} \dot{B} \dot{C} \dot{D}} \equiv \dot{\mathcal{D}}_{[\dot{A}}(\dot{\mathcal{P}}+\dot{\overline{\mathcal{P}}})_{\dot{B}] \dot{C} \dot{D}}+\dot{\mathcal{D}}_{[\dot{C}}(\dot{\mathcal{P}}+\dot{\overline{\mathcal{P}}})_{\dot{D}] \dot{A} \dot{B}}
$$

This shows that, in an identical manner to the untwisted case (2.66), $\dot{\mathcal{G}}_{\dot{A} \dot{B} \dot{C} \dot{D}}$ is still semi-covariant after the twist. The completely covariant index-two ("Ricci") and index-zero (scalar) twisted curvatures are as untwisted cases,

$$
\dot{\mathcal{G}}_{p r \bar{q}}{ }^{r}, \quad \dot{\mathcal{G}}_{p \bar{q} \bar{q}}{ }^{\bar{r}}, \quad \dot{\mathcal{G}}_{p q}^{p q}, \quad \dot{\mathcal{G}}_{\bar{p} \bar{q}} \bar{p} \bar{q} .
$$

Finally we look into $\dot{S}_{\dot{A} \dot{B} \dot{C} \dot{D}}$. It is straightforward to check that it is not semicovariant. It produces additional anomalous terms which are not governed by the 
index-six projectors, ${ }^{13}$

$$
\begin{aligned}
& \left(\delta_{\dot{X}}-\hat{\mathcal{L}}_{\dot{X}}\right) \dot{S}_{\dot{A} \dot{B} \dot{C} \dot{D}} \\
& =\dot{\nabla}_{[A}\left(\delta_{\dot{X}}-\hat{\mathcal{L}}_{\dot{X}}\right) \dot{\Gamma}_{\dot{B}] \dot{C} \dot{D}}+2 \dot{\nabla}_{[\dot{A}} \dot{D}_{\dot{B}]} \dot{D}_{[\dot{C}} X_{\dot{D}]}+\dot{D}_{\dot{E}} \dot{X}_{[\dot{A}} \dot{D}^{\dot{E}} \dot{\Gamma}_{\dot{B}] \dot{C} \dot{D}}+[(\dot{A}, \dot{B}) \leftrightarrow(\dot{C}, \dot{D})] \\
& \equiv \dot{\nabla}_{[A}(\dot{\mathcal{P}}+\dot{\overline{\mathcal{P}}})_{\dot{B}] \dot{C} \dot{D}}-\dot{D}_{[\dot{A}}\left(\Omega^{\dot{E}}{ }_{\dot{B}] \dot{K}} \Omega_{\dot{E} \dot{C} \dot{D}} \dot{X}^{\dot{K}}\right)+[(\dot{A}, \dot{B}) \leftrightarrow(\dot{C}, \dot{D})] .
\end{aligned}
$$

We conclude that $\dot{S}_{\dot{A} \dot{B} \dot{C} \dot{D}}$ is of no use in the twisted double field theory. We discard it and keep $\mathcal{G}_{\dot{A} \dot{B} \dot{C} \dot{D}}$ only.

- Identities which still hold after the twist.

Straightforward yet useful implications of the twistability conditions include

$$
\dot{D}_{\dot{A}} \dot{D}^{\dot{A}}-2 \dot{D}_{\dot{A}} \dot{d} \dot{D}^{\dot{A}} \equiv f^{\dot{A}} \dot{D}_{\dot{A}} \equiv 0, \quad \dot{\Gamma}_{p \bar{q}}^{\dot{q}_{\dot{C}}} \dot{\partial}_{\dot{C}} \equiv 0
$$

From (2.54), (3.52) and (3.62), the Bianchi identity of $\mathcal{G}_{\dot{A} \dot{B} \dot{C} \dot{D}}$ is valid upon the twistability conditions,

$$
\dot{\mathcal{G}}_{\dot{A}[\dot{B} \dot{C} \dot{D}]} \equiv 0 \text {. }
$$

Further from (2.55), it is straightforward to show

$$
\dot{\mathcal{G}}_{p q \bar{r} \bar{s}} \equiv \frac{3}{2} \Omega_{\dot{E} \dot{A}[\dot{B}} \Omega^{\dot{E}}{ }_{\dot{C} \dot{D}]} \dot{V}_{p}^{\dot{A}} \dot{V}^{\dot{B}}{ }_{q} \dot{\bar{V}}_{\bar{r}} \dot{\bar{V}}^{\dot{D}_{\bar{s}}} \equiv 0, \quad \dot{\mathcal{G}}_{p \bar{q} r \bar{s}} \equiv 0, \quad \dot{\mathcal{G}}_{p r \bar{q}}{ }^{r} \equiv \dot{\mathcal{G}}_{p \bar{r} \bar{q}} \bar{r} \equiv \frac{1}{2} \dot{\mathcal{G}}_{p \bar{q}},
$$

and notably,

$$
\dot{\mathcal{G}}_{p q}{ }^{p q}+\dot{\mathcal{G}}_{\bar{p} \bar{q}}^{\bar{p} \bar{q}} \equiv \frac{1}{6} f_{\dot{A} \dot{B} \dot{C}} f^{\dot{A} \dot{B} \dot{C}} .
$$

That is to say, replacing $\dot{S}_{\dot{A} \dot{B} \dot{C} \dot{D}}$ by $\dot{\mathcal{G}}_{\dot{A} \dot{B} \dot{C} \dot{D}}$, almost all the properties of the four-index curvature (2.55) still hold after the twist, up to the twistability conditions. The only exception is (3.88) and this is also crucial.

The relations between the completely covariant curvatures and the completely covariant derivatives (2.89), (2.95), (2.96) still hold after the twist,

$$
\begin{aligned}
\frac{1}{2}\left[\dot{\mathcal{D}}_{p}, \dot{\mathcal{D}}_{\bar{q}}\right] T^{p} & \equiv \dot{\mathcal{G}}_{p r \bar{q}}{ }^{r} T^{p}, & \frac{1}{2}\left[\dot{\mathcal{D}}_{p}, \dot{\mathcal{D}}_{\bar{q}}\right] T^{\bar{q}} & \equiv-\dot{\mathcal{G}}_{p \bar{r}}{ }^{\bar{q}} T^{\bar{q}}, \\
{\left[\gamma^{p} \dot{\mathcal{D}}_{p}, \dot{\mathcal{D}}_{\bar{q}}\right] \varepsilon } & \equiv \dot{\mathcal{G}}_{p r \bar{q}}{ }^{r} \gamma^{p} \varepsilon, & {\left[\dot{\mathcal{D}}_{p}, \bar{\gamma}^{\bar{q}} \dot{\mathcal{D}}_{\bar{q}}\right] \varepsilon^{\prime} } & \equiv-\dot{\mathcal{G}}_{p \bar{r} \bar{q}}{ }^{\bar{r}} \gamma^{\bar{q}} \varepsilon^{\prime}, \\
\left(\gamma^{p} \dot{\mathcal{D}}_{p}\right)^{2} \varepsilon+\dot{\mathcal{D}}_{\bar{p}} \dot{\mathcal{D}}^{\bar{p}} \varepsilon & \equiv-\frac{1}{4} \dot{\mathcal{G}}_{p q}^{p q} \varepsilon, & \left(\bar{\gamma}^{\bar{p}} \dot{\mathcal{D}}_{\bar{p}}\right)^{2} \varepsilon^{\prime}+\dot{\mathcal{D}}_{p} \dot{\mathcal{D}}^{p} \varepsilon^{\prime} & \equiv-\frac{1}{4} \dot{\mathcal{G}}_{\bar{p} \bar{q}}{ }^{\bar{p}} \varepsilon^{\prime}
\end{aligned}
$$

But, in contrast to (2.83), we get after the twist,

$$
\left(\dot{\mathcal{D}}_{ \pm}\right)^{2} \mathcal{T} \equiv-\frac{1}{24} f_{\dot{A} \dot{B} \dot{C}} f^{\dot{A} \dot{B} \dot{C}} \mathcal{T}
$$

\footnotetext{
${ }^{13}$ Putting the three relations (3.65), (3.66) and (3.82) together, we conjecture an equivalence relation,

$$
\dot{\mathcal{L}}_{\dot{X}}\left(\Omega_{\dot{E} \dot{A} \dot{B}} \Omega^{\dot{E}}{ }_{\dot{C} \dot{D}}\right) \equiv-2 \dot{D}_{[\dot{A}}\left(\Omega^{\dot{E}}{ }_{\dot{B}] \dot{K}} \Omega_{\dot{E} \dot{C} \dot{D}} \dot{X}^{\dot{K}}\right)-2 \dot{D}_{[\dot{C}}\left(\Omega^{\dot{E}}{ }_{\dot{D}] \dot{K}} \Omega_{\dot{E} \dot{A} \dot{B}} \dot{X}^{\dot{K}}\right),
$$

of which a direct proof is desirable.
} 
This indicates that in addition to the twistability conditions, in the presence of the $\mathrm{R}-\mathrm{R}$ sector, in order to ensure the nilpotency of the differential operators, $\dot{\mathcal{D}}_{ \pm}$, which should define the twisted R-R gauge symmetry or the 'twisted R-R cohomology' consistently, we should separately impose

$$
f_{\dot{A} \dot{B} \dot{C}} f^{\dot{A} \dot{B} \dot{C}} \equiv 0
$$

For a relevant previous work we refer the readers to [28] where the R-R sector was treated as an $\mathbf{O}(10,10)$ spinor which can be related to our treatment after the diagonal gauge fixing of the twofold local Lorentz symmetries [38]. We shall see shortly that this extra condition is also required for the supersymmetric invariance of the twisted maximal SDFT.

- "Effective connection" and internal coordinate independence.

Writing explicitly,

$$
\dot{D}_{\dot{A}}=\dot{\partial}_{\dot{A}}+\Omega_{\dot{A}}, \quad \dot{\nabla}_{\dot{A}}=\dot{\partial}_{\dot{A}}+\Omega_{\dot{A}}+\dot{\Gamma}_{\dot{A}},
$$

it may appear plausible to view $\Omega_{\dot{A}}+\dot{\Gamma}_{\dot{A}}$ as the "effective connection". Upon the twistability conditions (3.46)-(3.50), this "effective connection" reads explicitly, cf. [29],

$$
\begin{aligned}
& \Omega_{\dot{C} \dot{A} \dot{B}}+\dot{\Gamma}_{\dot{C} \dot{A} \dot{B}} \equiv 2\left(\dot{P} \dot{\partial}_{\dot{C}} \dot{P} \dot{\bar{P}}\right)_{[\dot{A} \dot{B}]}+2\left(\dot{\bar{P}}_{[\dot{A}} \dot{D} \dot{\bar{P}}_{\dot{B}]} \dot{E}-\dot{P}_{[\dot{A}} \dot{D}_{\dot{P}} \dot{P}_{\dot{B}]} \dot{E}^{2} \dot{\partial}_{\dot{D}} \dot{P}_{\dot{E} \dot{C}}\right. \\
& -\frac{4}{D-1}\left(\dot{\bar{P}}_{\dot{C}[\dot{A}} \dot{\bar{P}}_{\dot{B}]}^{\dot{D}}+\dot{P}_{\dot{C}[\dot{A}} \dot{P}_{\dot{B}]}^{\dot{D}}\right)\left(\dot{\partial}_{\dot{D}} \dot{d}+(\dot{P} \dot{\partial} \dot{E} \dot{P} \dot{\bar{P}})_{[\dot{E} \dot{D}]}\right) \\
& +\left(\dot{\bar{P}}_{\dot{C}} \dot{D}^{\dot{P}_{\dot{A}}} \dot{E}^{\dot{P}} \dot{P}_{\dot{B}} \dot{F}+\dot{P}_{\dot{C}} \dot{D} \dot{\bar{P}}_{\dot{A}} \dot{E} \dot{\bar{P}}_{\dot{B}} \dot{F}\right) f_{\dot{D} \dot{E} \dot{F}} \\
& +(\dot{\mathcal{P}}+\dot{\overline{\mathcal{P}}})_{\dot{C} \dot{A} \dot{B}} \dot{D} \dot{E} \dot{F} \Omega_{\dot{D} \dot{E} \dot{F}}
\end{aligned}
$$

In particular, we have

$$
\dot{V}^{\dot{A}}{ }_{p} \dot{V}^{\dot{B}}{ }_{q} \dot{V}^{\dot{C}}{ }_{r}\left(\Omega_{[\dot{A} \dot{B} \dot{C}]}+\dot{\Gamma}_{[\dot{A} \dot{B} \dot{C}]}\right)=\frac{1}{3} f_{p q r}, \quad \dot{\bar{V}}^{\dot{A}}{ }_{\bar{p}} \dot{\bar{V}}^{\dot{B}}{ }_{\bar{q}} \dot{\bar{V}}^{\dot{C}_{\bar{r}}}\left(\Omega_{[\dot{A} \dot{B} \dot{C}]}+\dot{\Gamma}_{[\dot{A} \dot{B} \dot{C}]}\right)=\frac{1}{3} f_{\bar{p} \bar{q} \bar{r}}
$$

We may view the last two lines of (3.93) as "effective torsions". They satisfy the desired properties (2.28). In this "effective" point of view, the "torsion" should be defined from the difference, $\hat{\mathcal{L}}_{X}(\dot{\nabla})-\hat{\mathcal{L}}_{X}(\dot{\partial})$, instead of the trivial one (3.59), $\hat{\mathcal{L}}_{X}(\dot{\nabla})-\hat{\mathcal{L}}_{X}(\dot{D})=0$. For further related discussion, we refer readers to section 3.4.2 of $[25]$.

We also note that only the last line, i.e. the six-index projection of $\Omega_{\dot{D} \dot{E} \dot{F}}$, may depend on the internal coordinates, which could be problematic. However, as seen in (3.94), it is easy to see that this potentially dangerous internal coordinate dependency disappears thoroughly inside the completely covariant derivatives, listed in (3.77), (3.78), (3.79) and (3.80). This cancellation further implies that the completely covariant curvatures (3.83) are also independent of the internal coordinates, 
since the completely covariant curvatures can be constructed from the quadratic completely covariant differential operators, see (3.89) with (3.80).

The above expression of the effective connection (3.93) is also comparable to the torsionful connection proposed by Berman and Lee in [29]. With the intention of handling the twisted generalized Lie derivative [27, 29], i.e. (3.54), they introduced torsions by clever guess work. Their torsionful connection differs from our effective connection (3.93). Yet, it nevertheless satisfies (3.94) and the difference amounts to certain six-index projection terms. Accordingly, their proposal is practically consistent with our result. A novel contribution of the present work is to derive the effective connection (3.93) straightforwardly by applying the U-twisting ansatz (3.3) to the semi-covariant formalism, without any ambiguity.

\section{Twisted supersymmetric double field theory}

Here we present explicitly half-maximal (i.e. sixteen) and maximal (i.e. thirty two) supersymmetric gauged double field theories as the twists of the previously constructed $\mathcal{N}=1$ and $\mathcal{N}=2, D=10$ supersymmetric double field theories. All the fields satisfy the twistability conditions, (3.46)-(3.50), and in the case of the maximal supersymmetric twist, one extra condition, i.e. (3.91) must be also met in order to ensure both the R-R gauge symmetry and the 32 supersymmetries unbroken.

\subsection{Half-maximal supersymmetric gauged double field theory}

After replacing $S_{A B C D}$ by $\mathcal{G}_{A B C D}$ and adding section-condition-vanishing purely bosonic terms of (2.75), we twist the $\mathcal{N}=1 D=10$ SDFT which was constructed in [36] to the full order in fermions. The twist leads to a half-maximal supersymmetric gauged double field theory of which the Lagrangian is

$$
\dot{\mathcal{L}}_{\text {Twisted SDFT }}^{\text {Half-maximal }}=e^{-2 \dot{d}}\left[\frac{1}{4} \dot{\mathcal{G}}_{p q}^{p q}+i \frac{1}{2} \bar{\rho} \gamma^{p} \dot{\mathcal{D}}_{p} \rho-i \bar{\psi} \overline{\bar{p}} \dot{\mathcal{D}}_{\bar{p}} \rho-i \frac{1}{2} \bar{\psi}^{\bar{p}} \gamma^{q} \dot{\mathcal{D}}_{q} \psi_{\bar{p}}\right] .
$$

Each term in the Lagrangian is completely covariant with respect to the twisted diffeomorphisms, (3.37) or (3.54), the $\mathbf{S p i n}(1,9) \times \mathbf{S} \operatorname{pin}(9,1)$ local Lorentz symmetries, and a subgroup of $\mathbf{O}(10,10)$ which preserves the structure constant, $f_{\dot{A} \dot{B} \dot{C}}$. Being completely covariant, each term is also independent of the internal coordinates.

The leading order half-maximal (i.e. sixteen) twisted supersymmetry transformation rules are, for the twisted bosons,

$$
\delta_{\varepsilon} \dot{d}=-i \frac{1}{2} \bar{\varepsilon} \rho, \quad \delta_{\varepsilon} \dot{V}_{A p}=-i \dot{\bar{V}} \bar{A} \overline{\bar{\varepsilon}} \gamma_{p} \psi_{\bar{q}}, \quad \delta_{\varepsilon} \dot{\bar{V}}_{A \bar{p}}=+i \dot{V}_{A}^{q} \bar{\varepsilon} \gamma_{q} \psi_{\bar{p}}
$$

and for the 'untwisted' fermions,

$$
\delta_{\varepsilon} \rho=-\gamma^{p} \dot{\mathcal{D}}_{p} \varepsilon, \quad \delta_{\varepsilon} \psi_{\bar{p}}=\dot{\mathcal{D}}_{\bar{p}} \varepsilon
$$


The supersymmetry works, as the induced leading order variation of the Lagrangian vanishes, up to total derivatives and the twistability conditions, thanks to (3.89),

$\delta_{\varepsilon} \dot{\mathcal{L}}_{\text {Twisted SDFT }}^{\text {Half-maximal }} \equiv-i e^{-2 \dot{d}} \bar{\rho}\left[\left(\gamma^{p} \dot{\mathcal{D}}_{p}\right)^{2}+\dot{\mathcal{D}}_{\bar{p}} \dot{\mathcal{D}}^{\bar{p}}+\frac{1}{4} \dot{\mathcal{G}}_{p q}^{p q}\right] \varepsilon+i e^{-2 \dot{d}} \bar{\psi}^{\bar{p}}\left[\dot{\mathcal{G}}_{\bar{p} r q}{ }^{r} \gamma^{q}+\left[\dot{\mathcal{D}}_{\bar{p}}, \gamma^{q} \dot{\mathcal{D}}_{q}\right]\right] \varepsilon \equiv 0$.

As discussed at the end of section 3.2, the leading order supersymmetric invariance is sufficient to guarantee the full order completion. Outsourcing from the full order untwisted $\mathcal{N}=1 D=10$ SDFT [36], we only need to add the quartic fermions therein to the twisted Lagrangian (4.1) and the cubic fermions to the twisted supersymmetry transformation rules for the fermions (4.3).

As in the untwisted SDFT [36], the conventional Rarita-Schwinger term is forbidden, and this is due to the hybrid nature of the gravitino indices, $\psi_{\bar{p}}^{\alpha}$ : one $\mathbf{S p i n}(9,1)$ vectorial and the other $\operatorname{Spin}(1,9)$ spinorial. Simply they cannot be mixed. Nonetheless, the $\mathcal{N}=1$ $D=10$ SDFT reduces consistently to the minimal supergravity in ten-dimensions after the diagonal gauge fixing, $\mathbf{S p i n}(1,9) \times \mathbf{S p i n}(9,1) \rightarrow \mathbf{S p i n}(1,9)_{D}$, see the appendix of [36] for details.

It is worth while to note from the $Z_{2}$ symmetry which exchanges the two spin groups, $\operatorname{Spin}(1,9) \leftrightarrow \operatorname{Spin}(9,1)$, there is a parallel formulation of the half-maximal SDFT,

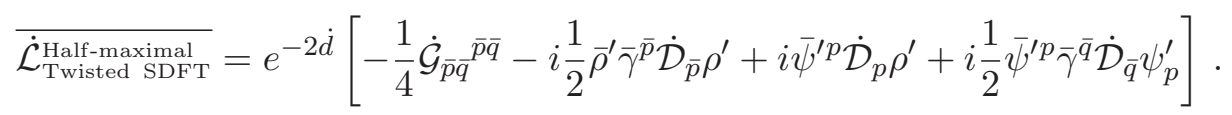

The supersymmetry is realized by

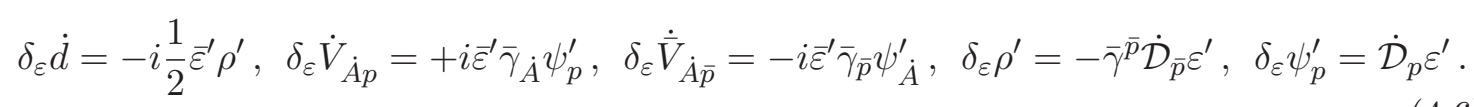

\subsection{Maximal supersymmetric gauged double field theory}

The twisting of the $\mathcal{N}=2 D=10$ SDFT which was constructed to the full order in fermions in [37], leads to the following maximal supersymmetric gauged double field theory Lagrangian,

$$
\begin{aligned}
\dot{\mathcal{L}}_{\text {Twisted SDFT }}^{\text {Maximal }}= & e^{-2 \dot{d}}\left[\frac{1}{8}\left(\dot{\mathcal{G}}_{p q}^{p q}-\dot{\mathcal{G}}_{\bar{p} \bar{q}}^{\bar{p}}\right)+\frac{1}{2} \operatorname{Tr}(\dot{\mathcal{F}} \overline{\dot{\mathcal{F}}})-i \bar{\rho} \dot{\mathcal{F}} \rho^{\prime}+i \bar{\psi}_{\bar{p}} \gamma_{q} \dot{\mathcal{F}} \bar{\gamma}^{\bar{p}} \psi^{\prime q}+i \frac{1}{2} \bar{\rho} \gamma^{p} \dot{\mathcal{D}}_{p} \rho\right. \\
& \left.-i \bar{\psi}^{\bar{p}} \dot{\mathcal{D}}_{\bar{p}} \rho-i \frac{1}{2} \bar{\psi}^{\bar{p}} \gamma^{q} \dot{\mathcal{D}}_{q} \psi_{\bar{p}}-i \frac{1}{2} \bar{\rho}^{\prime} \bar{\gamma}^{\bar{p}} \dot{\mathcal{D}}_{\bar{p}} \rho^{\prime}+i \bar{\psi}^{\prime p} \dot{\mathcal{D}}_{p} \rho^{\prime}+i \frac{1}{2} \bar{\psi}^{\prime p} \bar{\gamma}^{\bar{q}} \dot{\mathcal{D}}_{\bar{q}} \psi_{p}^{\prime}\right]
\end{aligned}
$$

As in the half-maximal case (4.1), each term in the Lagrangian is independent of the internal coordinates, and is completely covariant with respect to the twisted diffeomorphisms, the $\operatorname{Spin}(1,9) \times \mathbf{S p i n}(9,1)$ local Lorentz symmetries, the structure constant preserving subgroup of $\mathbf{O}(10,10)$, and further the R-R gauge symmetry provided the extra condition of (3.91),

$$
\delta \mathcal{C}=\dot{\mathcal{D}}_{+} \Lambda \longrightarrow \delta \dot{\mathcal{F}}=\left(\dot{\mathcal{D}}_{+}\right)^{2} \Lambda \equiv-\frac{1}{24} f_{\dot{A} \dot{B} \dot{C}} f^{\dot{A} \dot{B} \dot{C}} \Lambda \equiv 0
$$


The leading order maximal (i.e. thirty two) twisted supersymmetry transformation rules are, for the bosons,

$$
\begin{aligned}
& \delta_{\varepsilon} \dot{d}=-i \frac{1}{2}\left(\bar{\varepsilon} \rho+\bar{\varepsilon}^{\prime} \rho^{\prime}\right), \quad \delta_{\varepsilon} \dot{V}_{\dot{A} p}=i \dot{\bar{V}}_{\dot{A}}^{\bar{q}}\left(\bar{\varepsilon}^{\prime} \bar{\gamma} \bar{q} \psi_{p}^{\prime}-\bar{\varepsilon} \gamma_{p} \psi_{\bar{q}}\right), \quad \delta_{\varepsilon} \dot{\bar{V}}_{\dot{A} \bar{p}}=i \dot{V}_{\dot{A}}^{q}\left(\bar{\varepsilon} \gamma_{q} \psi_{\bar{p}}-\bar{\varepsilon}^{\prime} \bar{\gamma}_{\bar{p}} \psi_{q}^{\prime}\right), \\
& \delta_{\varepsilon} \mathcal{C}=i \frac{1}{2}\left(\gamma^{p} \varepsilon \bar{\psi}_{p}^{\prime}-\varepsilon \bar{\rho}^{\prime}-\psi_{\bar{p}} \bar{\varepsilon}^{\prime} \bar{\gamma}^{\bar{p}}+\rho \bar{\varepsilon}^{\prime}\right)+\mathcal{C} \delta_{\varepsilon} \dot{d}-\frac{1}{2}\left(\dot{\bar{V}}_{\bar{q}}^{\dot{A}} \delta_{\varepsilon} \dot{V}_{\dot{A} p}\right) \gamma^{(11)} \gamma^{p} \mathcal{C} \bar{\gamma}^{\bar{q}}
\end{aligned}
$$

and for the fermions,

$$
\delta_{\varepsilon} \rho=-\gamma^{p} \dot{\mathcal{D}}_{p} \varepsilon, \quad \delta_{\varepsilon} \rho^{\prime}=-\bar{\gamma}^{\bar{p}} \dot{\mathcal{D}}_{\bar{p}} \varepsilon^{\prime}, \quad \delta_{\varepsilon} \psi_{\bar{p}}=\dot{\mathcal{D}}_{\bar{p}} \varepsilon+\dot{\mathcal{F}} \bar{\gamma}_{\bar{p}} \varepsilon^{\prime}, \quad \delta_{\varepsilon} \psi_{p}^{\prime}=\dot{\mathcal{D}}_{p} \varepsilon^{\prime}+\overline{\mathcal{\mathcal { F }}} \gamma_{p} \varepsilon .
$$

Ignoring total derivatives and up to the twistability conditions, the supersymmetric infinitesimal variation of the Lagrangian is, from (3.87), (3.88), (3.89), (3.90) and the appendices of [37],

$$
\begin{aligned}
& \delta_{\varepsilon} \dot{\mathcal{L}}_{\text {Twisted SDFT }}^{\text {Maximal }} \equiv i \frac{1}{8} e^{-2 \dot{d}}\left(\bar{\rho} \varepsilon-\bar{\rho}^{\prime} \varepsilon^{\prime}\right)\left(\dot{\mathcal{G}}_{p q}^{p q}+\dot{\mathcal{G}}_{\bar{p} \bar{q}} \bar{q} \bar{q}\right)-i \frac{1}{2} e^{-2 \dot{d}}\left(\bar{\psi}^{\bar{q}} \gamma^{p} \varepsilon+\bar{\psi}^{\prime p} \bar{\gamma}^{\bar{q}} \varepsilon^{\prime}\right)\left(\dot{\mathcal{G}}_{p r \bar{q}}{ }^{r}-\dot{\mathcal{G}}_{p \bar{q}} \overline{\bar{q}}\right) \\
& +i \frac{1}{2} e^{-2 \dot{d}} \operatorname{Tr}\left[\left(\rho^{\prime} \bar{\varepsilon}+\psi_{p}^{\prime} \bar{\varepsilon} \gamma^{p}+\varepsilon^{\prime} \bar{\rho}+\bar{\gamma}^{\bar{p}} \varepsilon^{\prime} \bar{\psi}_{\bar{p}}\right)\left(\mathcal{D}_{+}\right)^{2} \mathcal{C}\right] \\
& +i \frac{1}{8} e^{-2 d}\left(\bar{\varepsilon} \gamma_{p} \psi_{\bar{q}}-\bar{\varepsilon}^{\prime} \bar{\gamma}_{\bar{q}} \psi_{p}^{\prime}\right) \operatorname{Tr}\left(\gamma^{p} \dot{\mathcal{F}}_{-} \bar{\gamma}^{\bar{q}} \dot{\mathcal{F}}_{-}\right) \\
& \equiv i \frac{1}{48} e^{-2 \dot{d}}\left(\bar{\rho} \varepsilon-\bar{\rho}^{\prime} \varepsilon^{\prime}+\bar{\varepsilon} \mathcal{C} \rho^{\prime}+\bar{\varepsilon} \gamma^{p} \mathcal{C} \psi_{p}^{\prime}+\bar{\rho} \mathcal{C} \varepsilon^{\prime}+\bar{\psi}_{\bar{p}} \mathcal{C} \bar{\gamma}^{\bar{p}} \varepsilon^{\prime}\right) \times f_{\dot{A} \dot{B} \dot{C}} f^{\dot{A} \dot{B} \dot{C}} \\
& +i \frac{1}{8} e^{-2 d}\left(\bar{\varepsilon} \gamma_{p} \psi_{\bar{q}}-\bar{\varepsilon}^{\prime} \bar{\gamma}_{\bar{q}} \psi_{p}^{\prime}\right) \operatorname{Tr}\left(\gamma^{p} \dot{\mathcal{F}}_{-} \bar{\gamma}^{\bar{q}} \overline{\dot{\mathcal{F}}_{-}}\right) \text {. }
\end{aligned}
$$

Here $\dot{\mathcal{F}}_{-}$denotes the (leading order) self-dual part of the R-R field strength, cf. (2.110),

$$
\dot{\mathcal{F}}_{-}:=\left(1-\gamma^{(11)}\right) \dot{\mathcal{F}}
$$

Thus, requiring the extra condition (3.91) which we recall here,

$$
f_{\dot{A} \dot{B} \dot{C}} f^{\dot{A} \dot{B} \dot{C}} \equiv 0
$$

the action is supersymmetric invariant modulo the self-duality, ${ }^{14}$ up to surface integrals. Once again, the leading order supersymmetric invariance guarantees the full order completion.

\subsection{Explicit comparison with the untwisted case}

To compare with the untwisted DFT and to identify the newly added terms after the U-twist, we dismantle the U-derivatives, $\dot{D}_{\dot{A}}$, explicitly and obtain up to the twistability

\footnotetext{
${ }^{14}$ For consistency, the supersymmetric variation of the self-duality relation is, even in the full order supersymmetric completion, precisely closed by the gravitino equations of motion [37].
} 
conditions,

$$
\begin{aligned}
& +\dot{\mathcal{G}}_{p q} p q \equiv \frac{1}{16} \dot{\mathcal{H}}^{\dot{A} \dot{B}} \dot{\partial}_{\dot{A}} \dot{\mathcal{H}}_{\dot{C} \dot{D}} \dot{\partial}_{\dot{B}} \dot{\mathcal{H}} \dot{C} \dot{D}+\frac{1}{4} \dot{\mathcal{H}}^{\dot{A} \dot{B}} \dot{\partial} \dot{C} \dot{\mathcal{H}}_{\dot{A} \dot{D}} \dot{\partial}^{\dot{D}} \dot{\mathcal{H}}_{\dot{B} \dot{C}}-\frac{1}{2} \dot{\partial}_{\dot{A}} \dot{\partial}_{\dot{B}} \dot{\mathcal{H}}^{\dot{A} \dot{B}} \\
& -2 \dot{\mathcal{H}}^{\dot{A} \dot{B}} \dot{\partial}_{\dot{A}} \dot{d} \dot{\partial}_{\dot{B}} \dot{d}+2 \dot{\mathcal{H}}^{\dot{A} \dot{B}} \dot{\partial}_{\dot{A}} \dot{\partial}_{\dot{B}} \dot{d}+2 \dot{\partial}_{\dot{A}} \dot{\mathcal{H}}^{\dot{A} \dot{B}} \dot{\partial}_{\dot{B}} \dot{d}
\end{aligned}
$$

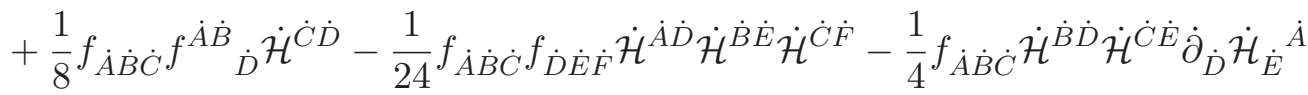

$$
\begin{aligned}
& +\frac{1}{12} f_{\dot{A} \dot{B} \dot{C}} f^{\dot{A} \dot{B} \dot{C}}
\end{aligned}
$$

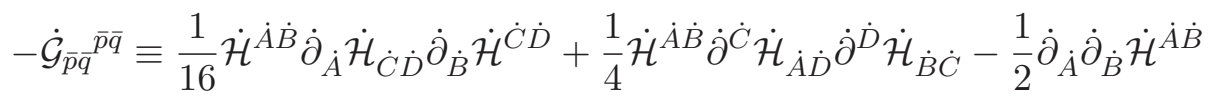

$$
\begin{aligned}
& -2 \dot{\mathcal{H}} \dot{\dot{A}} \dot{B} \dot{\partial}_{\dot{A}} \dot{d} \dot{\partial}_{\dot{B}} \dot{d}+2 \dot{\mathcal{H}}^{\dot{A} \dot{B}} \dot{\partial}_{\dot{A}} \dot{\partial}_{\dot{B}} \dot{d}+2 \dot{\partial}_{\dot{A}} \dot{\mathcal{H}}^{\dot{A} \dot{B}} \dot{\partial}_{\dot{B}} \dot{d} \\
& +\frac{1}{8} f_{\dot{A} \dot{B} \dot{C}} f^{\dot{A} \dot{B}}{ }_{\dot{D}} \dot{\mathcal{H}} \dot{C} \dot{D}-\frac{1}{24} f_{\dot{A} \dot{B} \dot{C}} f_{\dot{D} \dot{E} \dot{F}} \dot{\mathcal{H}}^{\dot{A} \dot{D}} \dot{\mathcal{H}}^{\dot{B} \dot{E}} \dot{\mathcal{H}} \dot{C} \dot{F}-\frac{1}{4} f_{\dot{A} \dot{B} \dot{C}} \dot{\mathcal{H}}^{\dot{B} \dot{D}} \dot{\mathcal{H}} \dot{C} \dot{E} \dot{\partial}_{\dot{D}} \dot{\mathcal{H}}_{\dot{E}}{ }^{\dot{A}} \\
& -\frac{1}{12} f_{\dot{A} \dot{B} \dot{C}} f^{\dot{A} \dot{B} \dot{C}} \text {. }
\end{aligned}
$$

It follows that the sum, $\dot{\mathcal{G}}_{p q}^{p q}+\dot{\mathcal{G}}_{\bar{p} \bar{q}}^{\bar{p} \bar{q}}=\frac{1}{6} f_{\dot{A} \dot{B} \dot{C}} f^{\dot{A} \dot{B} \dot{C}}$, indeed gives (3.88), and the difference reads

$$
\begin{aligned}
& \dot{\mathcal{G}}_{p q}{ }^{p q}-\dot{\mathcal{G}}_{\bar{p} \bar{q}} \bar{p} \bar{q} \\
& \equiv \frac{1}{8} \dot{\mathcal{H}}^{\dot{A} \dot{B}} \dot{\partial}_{\dot{A}} \dot{\mathcal{H}}_{\dot{C} \dot{D}} \dot{\partial}_{\dot{B}} \dot{\mathcal{H}} \dot{C} \dot{D}+\frac{1}{2} \dot{\mathcal{H}}^{\dot{A} \dot{B}} \dot{\partial} \dot{C} \dot{\mathcal{H}}_{\dot{A} \dot{D}} \dot{\partial}^{\dot{D}} \dot{\mathcal{H}}_{\dot{B} \dot{C}}-\dot{\partial}_{\dot{A}} \dot{\partial}_{\dot{B}} \dot{\mathcal{H}}^{\dot{A} \dot{B}} \\
& -4 \dot{\mathcal{H}}^{\dot{A} \dot{B}} \dot{\partial}_{\dot{A}} \dot{d} \dot{\partial}_{\dot{B}} \dot{d}+4 \dot{\mathcal{H}}^{\dot{A} \dot{B}} \dot{\partial}_{\dot{A}} \dot{\partial}_{\dot{B}} \dot{d}+4 \dot{\partial}_{\dot{A}} \dot{\mathcal{H}}^{\dot{A} \dot{B}} \dot{\partial}_{\dot{B}} \dot{d} \\
& +\frac{1}{4} f_{\dot{A} \dot{B} \dot{C}} f^{\dot{A} \dot{B}} \dot{D}^{\dot{\mathcal{H}} \dot{C} \dot{D}}-\frac{1}{12} f_{\dot{A} \dot{B} \dot{C}} f_{\dot{D} \dot{E} \dot{F}} \dot{\mathcal{H}}^{\dot{A} \dot{D}} \dot{\mathcal{H}}^{\dot{B} \dot{E}} \dot{\mathcal{H}} \dot{C} \dot{F}-\frac{1}{2} f_{\dot{A} \dot{B} \dot{C}} \dot{\mathcal{H}}^{\dot{B} \dot{D}} \dot{\mathcal{H}}^{\dot{C}} \dot{E} \dot{\partial}_{\dot{D}} \dot{\mathcal{H}}_{\dot{E}}{ }^{\dot{A}} .
\end{aligned}
$$

In the above, i.e. (4.14) and (4.15), the first two lines on the right hand sides essentially correspond to the original untwisted DFT Lagrangian [4] i.e. (2.98) written in terms of the generalized metric. The third line then matches with the literature $[21,22,27,29,50]$. The last lines in (4.14) correspond to the DFT cosmological constant [35] which is apparently the special feature of the half-maximal supersymmetric DFT [21, 22, 27, 29]. Depending on the choice of $+\dot{\mathcal{G}}_{p q} p q$ or $-\dot{\mathcal{G}}_{\bar{p}} \bar{p} \bar{q}$ we may freely fix the sign of it.

It is further worth while to note

$$
\begin{aligned}
& \dot{\mathcal{F}}=\dot{\mathcal{D}}_{+} \mathcal{C}=\left.\dot{\mathcal{D}}_{+} \mathcal{C}\right|_{\dot{D}} \equiv\left.\dot{\mathcal{D}}_{+} \mathcal{C}\right|_{\dot{\partial}}+\frac{1}{12} f_{p q r} \gamma^{p q r} \mathcal{C}-\frac{1}{4} f_{p \bar{q} \bar{r}} \gamma^{p} \mathcal{C} \bar{\gamma}^{\bar{q} \bar{r}} \\
&-\frac{1}{12} f_{\bar{p} \bar{q} r} \gamma^{(11)} \mathcal{C} \bar{\gamma}^{\bar{p} \bar{r}}+\frac{1}{4} f_{p q \bar{r}} \gamma^{(11)} \gamma^{p q} \mathcal{C} \bar{\gamma}^{\bar{r}} \\
& \gamma^{p} \dot{\mathcal{D}}_{p} \rho=\left.\left.\gamma^{p} \dot{\mathcal{D}}_{p} \rho\right|_{\dot{D}} \equiv \gamma^{p} \dot{\mathcal{D}}_{p} \rho\right|_{\dot{\partial}}+\frac{1}{12} f_{p q r} \gamma^{p q r} \rho \\
& \dot{\mathcal{D}}_{\bar{p}} \rho=\left.\left.\dot{\mathcal{D}}_{\bar{p} \rho}\right|_{\dot{D}} \equiv \dot{\mathcal{D}}_{\bar{p}} \rho\right|_{\dot{\partial}}+\frac{1}{4} f_{\bar{p} q r} \gamma^{q r} \rho \\
& \gamma^{q} \dot{\mathcal{D}}_{q} \psi_{\bar{p}}=\left.\left.\gamma^{q} \dot{\mathcal{D}}_{q} \psi_{\bar{p}}\right|_{\dot{D}} \equiv \gamma^{q} \dot{\mathcal{D}}_{q} \psi_{\bar{p}}\right|_{\dot{\partial}}+\frac{1}{12} f_{q r s} \gamma^{q r s} \psi_{\bar{p}}+f_{r \bar{p} \bar{q}} \gamma^{r} \psi^{\bar{q}},
\end{aligned}
$$


and

$$
\begin{aligned}
\bar{\gamma}^{\bar{p}} \dot{\mathcal{D}}_{\bar{p}} \rho^{\prime}=\left.\left.\bar{\gamma}^{\bar{p}} \dot{\mathcal{D}}_{\bar{p}} \rho^{\prime}\right|_{\dot{D}} \equiv \bar{\gamma}^{\bar{p}} \dot{\mathcal{D}}_{\bar{p}} \rho^{\prime}\right|_{\dot{\partial}}+\frac{1}{12} f_{\bar{p} \bar{q} \bar{r}} \bar{\gamma}^{\bar{p} \bar{q} \bar{r}} \rho^{\prime}, \\
\dot{\mathcal{D}}_{p} \rho^{\prime}=\left.\left.\dot{\mathcal{D}}_{p} \rho^{\prime}\right|_{\dot{D}} \equiv \dot{\mathcal{D}}_{p} \rho^{\prime}\right|_{\dot{\partial}}+\frac{1}{4} f_{p \bar{p} \bar{r}} \bar{\gamma}^{\bar{q} \bar{r}} \rho^{\prime} \\
\bar{\gamma}^{\bar{q}} \dot{\mathcal{D}}_{\bar{q}} \psi_{p}^{\prime}=\left.\left.\bar{\gamma}^{\bar{q}} \dot{\mathcal{D}}_{\bar{q}} \psi_{p}^{\prime}\right|_{\dot{D}} \equiv \bar{\gamma}^{\bar{q}} \dot{\mathcal{D}}_{\bar{q}} \psi_{p}^{\prime}\right|_{\dot{\partial}}+\frac{1}{12} f_{\bar{q} \bar{r} \bar{s}} \bar{\gamma}^{\bar{q} \bar{r} \bar{s}} \psi_{p}^{\prime}+f_{\bar{r} p q} \bar{\gamma}^{\bar{r}} \psi^{\prime q}
\end{aligned}
$$

As expected from the consistency of the "effective connection", (4.17) and (4.18) agree with Berman and Lee [29], while (4.16) is a new result we report in this work.

\section{Discussion}

In this paper, we have successfully twisted the semi-covariant formulations of the $\mathcal{N}=2$ and the $\mathcal{N}=1, D=10$ SDFT constructed in [36,37], and systematically derived the gauged maximal and half-maximal supersymmetric double field theories, (4.1) (4.5), (4.7), along with their supersymmetry transformation rules, (4.2), (4.3), (4.9), (4.5), (4.10). Our derivation is systematic in the sense that, we only applied the twisting ansatz (3.3) to the untwisted SDFT of [36, 37], and then without any ambiguity the gauged supersymmetric double field theories were straightforwardly derived. Further, just like the untwisted SDFT yet now subject to the twistability conditions, (3.46)-(3.50) and also (3.91) for the maximal supersymmetric twist, each term in the constructed Lagrangian is completely covariant. Namely, the NS-NS curvature term, the fermionic kinetic terms and the R-R kinetic term are all completely covariant, with respect to the twisted diffeomorphisms, the $\operatorname{Spin}(1,9) \times$ $\operatorname{Spin}(9,1)$ local Lorentz symmetries, the R-R gauge symmetry for the maximal case, and a subgroup of $\mathbf{O}(10,10)$ which preserves the structure constant. The twofold Lorentz symmetries are 'local' with respect to the dimensionally reduced external spacetime. The twisted and hence gauged SDFTs are completely fixed by requiring the supersymmetry to be unbroken, in the precisely same manner as the untwisted SDFTs.

The nilpotency of the twisted R-R cohomology differential operators (3.90), (3.91), implies the Bianchi identity for the twisted R-R flux,

$$
\dot{\mathcal{D}}_{+} \dot{\mathcal{F}}=\left(\dot{\mathcal{D}}_{+}\right)^{2} \mathcal{C} \equiv 0
$$

As demonstrated in the section 4.3 of [38], one may take the diagonal gauge fixing of the local Lorentz symmetry, expand the R-R potential in terms of the conventional $p$-form fields coupled to gamma matrices in a 'democratic' manner [51], and compute the R-R field strengths explicitly. The above Bianchi identity is then naturally expected to produce the 'tensor hierarchy' [52-54].

It is worth while to note that, while the twist breaks the $\mathbf{O}(10,10)$ T-duality to its subgroup which preserves the structure constant, $f_{\dot{A} \dot{B} \dot{C}}$, the $\mathbf{S p i n}(1,9) \times \mathbf{S p i n}(9,1)$ local Lorentz symmetries are still all unbroken after the twist and the dimensional reduction.

When the twisting data, $U_{A}^{\dot{A}}, \lambda$, do not satisfy the original section condition, the corresponding background cannot be identified as a solution to the untwisted ' $D=10$ ' supersymmetric double field theories. This might well motivate one to wonder about the 
existence of unknown genuinely ten-dimensional "generalized double field theory" with "relaxed" section conditions. However, the twistability conditions seem to admit only lower dimensional sections, as the non-trivial solutions. In those lower dimensions, the standard section condition must be obeyed, see (3.46), and its doubled coordinates are still to be gauged. We regard the twist not as an indication of the existence of any unknown $D=10$ "generalized DFT" but as a lower dimensional deformation of the known rigid untwisted $D=10$ theories, i.e. [36, 37]. A well known such example is the massive supersymmetric deformations of the super Yang-Mills quantum mechanics $[55,56]$. The deformations do not necessarily mean that the parental super Yang-Mills field theories can be likely deformed.

In this work, the R-R sector is taken as $\mathbf{O}(10,10)$ singlet and assumes the $\mathbf{S p i n}(1,9) \times$ $\operatorname{Spin}(9,1)$ local Lorentz bi-spinorial representation [25, 26, 37, 38, 57-60]. ${ }^{15}$ This made the twisting of the R-R sector rather trivial. Essentially, the R-R potential, $\mathcal{C}^{\alpha}{ }_{\bar{\alpha}}$, is not twisted, like other fermions. Only the R-R field strength, $\dot{\mathcal{F}}=\dot{\mathcal{D}}_{+} \mathcal{C}$, is influenced by the twist through the twisted nilpotent differential operator. We expect that this feature should change when the U-duality group is twisted in $\mathcal{M}$-theory setup, but this goes beyond the scope of the present work.

\section{Acknowledgments}

We wish to thank David Geissbühler, Kanghoon Lee and Diego Marqués for helpful discussions. This work was supported by the Fundación Séneca - Talento Investigador Program, and also by the National Research Foundation of Korea (NRF) with the Grants, 2012R1A2A2A02046739, 2013R1A1A1A05005747, 2015K1A3A1A21000302.

Open Access. This article is distributed under the terms of the Creative Commons Attribution License (CC-BY 4.0), which permits any use, distribution and reproduction in any medium, provided the original author(s) and source are credited.

\section{References}

[1] W. Siegel, Superspace duality in low-energy superstrings, Phys. Rev. D 48 (1993) 2826 [hep-th/9305073] [INSPIRE].

[2] C.M. Hull and B. Zwiebach, Double Field Theory, JHEP 09 (2009) 099 [arXiv:0904.4664] [INSPIRE].

[3] O. Hohm, C.M. Hull and B. Zwiebach, Background independent action for double field theory, JHEP 07 (2010) 016 [arXiv: 1003. 5027] [INSPIRE].

[4] O. Hohm, C.M. Hull and B. Zwiebach, Generalized metric formulation of double field theory, JHEP 08 (2010) 008 [arXiv: 1006 .4823] [INSPIRE].

[5] M.J. Duff, Duality Rotations in String Theory, Nucl. Phys. B 335 (1990) 610 [InSPIRE].

\footnotetext{
${ }^{15}$ Alternative approach puts the R-R sector into the $\mathbf{O}(10,10)$ spinorial representation [58, 61, 62], to which the bi-spinorial approach actually reduces after taking the diagonal gauge fixing, $\operatorname{Spin}(1,9) \times$ $\operatorname{Spin}(9,1) \rightarrow \operatorname{Spin}(1,9)_{D}$ [38]. Yet, it is not clear how to twist the $\mathbf{O}(10,10)$ spinor and then couple to the $\operatorname{Spin}(1,9)$ or $\operatorname{Spin}(9,1)$ fermions, cf. [21, 28].
} 
[6] A.A. Tseytlin, Duality Symmetric Formulation of String World Sheet Dynamics, Phys. Lett. B 242 (1990) 163 [inSPIRE].

[7] A.A. Tseytlin, Duality symmetric closed string theory and interacting chiral scalars, Nucl. Phys. B 350 (1991) 395 [inSPIRE].

[8] J.-H. Park, Comments on double field theory and diffeomorphisms, JHEP 06 (2013) 098 [arXiv: 1304.5946] [INSPIRE].

[9] O. Hohm and B. Zwiebach, Large Gauge Transformations in Double Field Theory, JHEP 02 (2013) 075 [arXiv: 1207.4198] [INSPIRE].

[10] K. Lee and J.-H. Park, Covariant action for a string in "doubled yet gauged" spacetime, Nucl. Phys. B 880 (2014) 134 [arXiv:1307.8377] [INSPIRE].

[11] O. Hohm, D. Lüst and B. Zwiebach, The Spacetime of Double Field Theory: Review, Remarks and Outlook, Fortsch. Phys. 61 (2013) 926 [arXiv:1309.2977] [INSPIRE].

[12] D.S. Berman, M. Cederwall and M.J. Perry, Global aspects of double geometry, JHEP 09 (2014) 066 [arXiv:1401.1311] [InSPIRE].

[13] C.M. Hull, Finite Gauge Transformations and Geometry in Double Field Theory, JHEP 04 (2015) 109 [arXiv: 1406.7794] [INSPIRE].

[14] U. Naseer, A note on large gauge transformations in double field theory, JHEP 06 (2015) 002 [arXiv:1504.05913] [INSPIRE].

[15] C.M. Hull, Global aspects of T-duality, gauged $\sigma$-models and T-folds, JHEP 10 (2007) 057 [hep-th/0604178] [INSPIRE].

[16] C.M. Hull, Doubled Geometry and T-Folds, JHEP 07 (2007) 080 [hep-th/0605149] [INSPIRE].

[17] D.S. Berman and D.C. Thompson, Duality Symmetric String and M-theory, Phys. Rept. 566 (2014) 1 [arXiv:1306.2643] [INSPIRE].

[18] B. de Wit, H. Samtleben and M. Trigiante, Magnetic charges in local field theory, JHEP 09 (2005) 016 [hep-th/0507289] [INSPIRE].

[19] J. Scherk and J.H. Schwarz, How to Get Masses from Extra Dimensions, Nucl. Phys. B 153 (1979) 61 [InSPIRE].

[20] N. Kaloper and R.C. Myers, The Odd story of massive supergravity, JHEP 05 (1999) 010 [hep-th/9901045] [INSPIRE].

[21] D. Geissbuhler, Double Field Theory and $N=4$ Gauged Supergravity, JHEP 11 (2011) 116 [arXiv: 1109.4280] [INSPIRE].

[22] G. Aldazabal, W. Baron, D. Marques and C. Núñez, The effective action of Double Field Theory, JHEP 11 (2011) 052 [Erratum ibid. 11 (2011) 109] [arXiv:1109.0290] [INSPIRE].

[23] J. Schon and M. Weidner, Gauged $N=4$ supergravities, JHEP 05 (2006) 034 [hep-th/0602024] [INSPIRE].

[24] G. Dibitetto, J.J. Fernandez-Melgarejo, D. Marques and D. Roest, Duality orbits of non-geometric fluxes, Fortsch. Phys. 60 (2012) 1123 [arXiv: 1203.6562] [INSPIRE].

[25] A. Coimbra, C. Strickland-Constable and D. Waldram, Supergravity as Generalised Geometry I: Type II Theories, JHEP 11 (2011) 091 [arXiv:1107.1733] [INSPIRE]. 
[26] A. Coimbra, C. Strickland-Constable and D. Waldram, Generalised Geometry and type-II Supergravity, Fortsch. Phys. 60 (2012) 982 [arXiv:1202.3170] [INSPIRE].

[27] M. Graña and D. Marques, Gauged Double Field Theory, JHEP 04 (2012) 020 [arXiv:1201.2924] [INSPIRE].

[28] D. Geissbuhler, D. Marques, C. Núñez and V. Penas, Exploring Double Field Theory, JHEP 06 (2013) 101 [arXiv: 1304.1472] [INSPIRE].

[29] D.S. Berman and K. Lee, Supersymmetry for Gauged Double Field Theory and Generalised Scherk-Schwarz Reductions, Nucl. Phys. B 881 (2014) 369 [arXiv: 1305.2747] [INSPIRE].

[30] D.S. Berman, C.D.A. Blair, E. Malek and M.J. Perry, The $O_{D, D}$ geometry of string theory, Int. J. Mod. Phys. A 29 (2014) 1450080 [arXiv:1303.6727] [InSPIRE].

[31] G. Aldazabal, D. Marques and C. Núñez, Double Field Theory: A Pedagogical Review, Class. Quant. Grav. 30 (2013) 163001 [arXiv:1305.1907] [INSPIRE].

[32] O. Hohm and S.K. Kwak, Frame-like Geometry of Double Field Theory, J. Phys. A 44 (2011) 085404 [arXiv:1011.4101] [InSPIRE].

[33] O. Hohm and S.K. Kwak, $N=1$ Supersymmetric Double Field Theory, JHEP 03 (2012) 080 [arXiv:1111.7293] [INSPIRE].

[34] I. Jeon, K. Lee and J.-H. Park, Differential geometry with a projection: Application to double field theory, JHEP 04 (2011) 014 [arXiv: 1011.1324] [INSPIRE].

[35] I. Jeon, K. Lee and J.-H. Park, Stringy differential geometry, beyond Riemann, Phys. Rev. D 84 (2011) 044022 [arXiv: 1105.6294] [INSPIRE].

[36] I. Jeon, K. Lee and J.-H. Park, Supersymmetric Double Field Theory: Stringy Reformulation of Supergravity, Phys. Rev. D 85 (2012) 081501 [Erratum ibid. D 86 (2012) 089903] [arXiv: 1112.0069] [INSPIRE].

[37] I. Jeon, K. Lee, J.-H. Park and Y. Suh, Stringy Unification of Type IIA and IIB Supergravities under $N=2 D=10$ Supersymmetric Double Field Theory, Phys. Lett. B 723 (2013) 245 [arXiv:1210.5078] [INSPIRE].

[38] I. Jeon, K. Lee and J.-H. Park, Ramond-Ramond Cohomology and $O(D, D) T$-duality, JHEP 09 (2012) 079 [arXiv: 1206.3478] [INSPIRE].

[39] M. Gualtieri, Generalized complex geometry, math.DG/0401221.

[40] M. Graña, R. Minasian, M. Petrini and D. Waldram, T-duality, Generalized Geometry and Non-Geometric Backgrounds, JHEP 04 (2009) 075 [arXiv: 0807.4527] [INSPIRE].

[41] C.M. Hull and B. Zwiebach, The Gauge algebra of double field theory and Courant brackets, JHEP 09 (2009) 090 [arXiv:0908.1792] [INSPIRE].

[42] I. Jeon, K. Lee and J.-H. Park, Incorporation of fermions into double field theory, JHEP 11 (2011) 025 [arXiv:1109.2035] [INSPIRE].

[43] D. Andriot and A. Betz, $\beta$-supergravity: a ten-dimensional theory with non-geometric fluxes and its geometric framework, JHEP 12 (2013) 083 [arXiv: 1306.4381] [INSPIRE].

[44] D. Andriot and A. Betz, Supersymmetry with non-geometric fluxes, or a $\beta$-twist in Generalized Geometry and Dirac operator, JHEP 04 (2015) 006 [arXiv:1411.6640] [INSPIRE]. 
[45] O. Hohm and B. Zwiebach, On the Riemann Tensor in Double Field Theory, JHEP 05 (2012) 126 [arXiv:1112.5296] [INSPIRE].

[46] I. Jeon, K. Lee and J.-H. Park, Double field formulation of Yang-Mills theory, Phys. Lett. B 701 (2011) 260 [arXiv:1102.0419] [InSPIRE].

[47] K. Peeters, A Field-theory motivated approach to symbolic computer algebra, Comput. Phys. Commun. 176 (2007) 550 [cs/0608005] [INSPIRE].

[48] K. Peeters, Introducing Cadabra: A Symbolic computer algebra system for field theory problems, hep-th/0701238 [INSPIRE].

[49] M. Garcia-Fernandez, Torsion-free generalized connections and Heterotic Supergravity, Commun. Math. Phys. 332 (2014) 89 [arXiv:1304.4294] [INSPIRE].

[50] O. Hohm and S.K. Kwak, Double Field Theory Formulation of Heterotic Strings, JHEP 06 (2011) 096 [arXiv:1103.2136] [INSPIRE].

[51] E. Bergshoeff, R. Kallosh, T. Ortín, D. Roest and A. Van Proeyen, New formulations of $D=10$ supersymmetry and D8-O8 domain walls, Class. Quant. Grav. 18 (2001) 3359 [hep-th/0103233] [INSPIRE].

[52] E.A. Bergshoeff, J. Hartong, O. Hohm, M. Huebscher and T. Ortín, Gauge Theories, Duality Relations and the Tensor Hierarchy, JHEP 04 (2009) 123 [arXiv:0901.2054] [INSPIRE].

[53] J.J. Fernandez-Melgarejo, T. Ortín and E. Torrente-Lujan, Maximal Nine Dimensional Supergravity, General gaugings and the Embedding Tensor, Fortsch. Phys. 60 (2012) 1012 [arXiv: 1209.3774] [INSPIRE].

[54] J.J. Fernandez-Melgarejo, Gaugings and other aspects in supergravity, arXiv:1311.7145 [INSPIRE].

[55] D.E. Berenstein, J.M. Maldacena and H.S. Nastase, Strings in flat space and pp waves from $N=4$ super Yang-Mills, JHEP 04 (2002) 013 [hep-th/0202021] [INSPIRE].

[56] N. Kim and J.-H. Park, Massive super Yang-Mills quantum mechanics: Classification and the relation to supermembrane, Nucl. Phys. B 759 (2006) 249 [hep-th/0607005] [INSPIRE].

[57] S.F. Hassan, T duality, space-time spinors and RR fields in curved backgrounds, Nucl. Phys. B 568 (2000) 145 [hep-th/9907152] [INSPIRE].

[58] S.F. Hassan, $\mathbf{S O}(d, d)$ transformations of Ramond-Ramond fields and space-time spinors, Nucl. Phys. B 583 (2000) 431 [hep-th/9912236] [INSPIRE].

[59] S.F. Hassan, Supersymmetry and the systematics of T duality rotations in type-II superstring theories, Nucl. Phys. Proc. Suppl. 102 (2001) 77 [hep-th/0103149] [InSPIRE].

[60] N. Berkovits and P.S. Howe, Ten-dimensional supergravity constraints from the pure spinor formalism for the superstring, Nucl. Phys. B 635 (2002) 75 [hep-th/0112160] [INSPIRE].

[61] M. Fukuma, T. Oota and H. Tanaka, Comments on T dualities of Ramond-Ramond potentials on tori, Prog. Theor. Phys. 103 (2000) 425 [hep-th/9907132] [INSPIRE].

[62] O. Hohm, S.K. Kwak and B. Zwiebach, Unification of Type II Strings and T-duality, Phys. Rev. Lett. 107 (2011) 171603 [arXiv:1106.5452] [INSPIRE]. 